\title{
Stabilisation of Antithetic Control via Molecular Buffering
}

\author{
Edward J. Hancock ${ }^{1,2}$ and Diego A. Oyarzún ${ }^{3,4}$ \\ 1) School of Mathematics and Statistics, The University of Sydney, NSW, 2006, Australia \\ ${ }^{2)}$ Charles Perkins Centre, The University of Sydney, NSW, 2006, Australia \\ 3) School of Informatics, The University of Edinburgh, United Kingdom \\ 4) School of Biological Sciences, The University of Edinburgh, United Kingdom
}

(Dated: 29 September 2021)

A key goal in synthetic biology is the construction of molecular circuits that robustly adapt to perturbations. Although many natural systems display perfect adaptation, whereby stationary molecular concentrations are insensitive to perturbations, its de novo engineering has proven elusive. The discovery of the antithetic control motif was a significant step toward a universal mechanism for engineering perfect adaptation. Antithetic control provides perfect adaptation in a wide range of systems, but it can lead to oscillatory dynamics due to loss of stability, and moreover, it can lose perfect adaptation in fast growing cultures. Here, we introduce an extended antithetic control motif that resolves these limitations. We show that molecular buffering, a widely conserved mechanism for homeostatic control in nature, stabilises oscillations and allows for near-perfect adaptation during rapid growth. We study multiple buffering topologies and compare their performance in terms of their stability and adaptation properties. We illustrate the benefits of our proposed strategy in exemplar models for biofuel production and growth rate control in bacterial cultures. Our results provide an improved circuit for robust control of biomolecular systems.

\section{INTRODUCTION}

Synthetic biology promises to revolutionise many sectors such as healthcare, chemical manufacture and materials engineering ${ }^{7}$. A number of such applications require precise control of biomolecular processes in face of environmental perturbations and process variability ${ }^{22}$. An important requirement in such control systems is perfect adaptation, a property whereby chemical concentrations remain insensitive to perturbations ${ }^{20,23}$. The molecular mechanisms that can produce perfect adaptation has been extensively studied in natural systems $3,14,20,23$. In these systems, perfect adaptation can be produced by a range of feedforward and feedback mechanisms ${ }^{3,20}$. Such natural systems have been shaped by evolutionary processes, but it remains unclear if they are sufficiently robust and tuneable for de novo engineering of perfect adaptation in synthetic circuits.

One approach to engineer perfect adaptation relies on the use of feedback control. As illustrated in Figure $1 \mathrm{~A}$, this strategy requires circuits that sense the output and act upon the inputs of a biomolecular process. The groundbreaking work by Briat and colleagues ${ }^{1,4}$ identified antithetic feedback as a promising candidate for engineering perfect adaptation in living systems. Antithetic control involves a feedback mechanism with two molecular components that sequester and annihilate each other (see Figure 1B). It enables a system output to robustly follow an input signal and remain insensitive to various types of perturbations, akin to what integral feedback achieves in classic control engineering strategies ${ }^{2}$.

The original antithetic control motif, however, has two weaknesses that can limit its applicability: it is of- ten not effective when cells are growing rapidly, and the feedback mechanism can cause unwanted oscillations under a range of conditions ${ }^{27}$. Specifically, dilution effects caused by cell growth cause "leaky integration" - so called because integration is a form of memory and dilution causes that memory to leak over time ${ }^{29}$. This prevents perfect adaptation from occurring during rapid growth. Although in some motif configurations, the loss of perfect adaptation can be partly mitigated with a stronger feedback ${ }^{29}$, in general the use of strong feedback results in the loss of stability and undesirable oscillations ${ }^{27}$. Such oscillations can be stabilised in specific motifs ${ }^{15}$, and in more general cases the combination of antithetic control with classic ProportionalIntegral-Derivative (PID) control has been shown to improve temporal regulation ${ }^{5,9}$. Yet to date, there is no general strategy to avoid oscillations and prevent the loss of adaptation during rapid growth.

Here we propose an extended antithetic control system that resolves the above limitations. We show that the addition of molecular buffers improves stability and suppresses undesirable oscillations, and moreover it can allow for near-perfect adaptation in fast growth regimes. Molecular buffering is a widespread regulatory mechanism in nature (e.g. ATP, calcium \& pH buffers ${ }^{17,21,32}$ ) that has received modest attention in the literature as compared to other regulatory mechanisms. Recent work found that the combination of buffering and feedback is often critical for robust regulation ${ }^{16,17}$. Buffering has the ability to attenuate fast disturbances and stabilise feedback control ${ }^{17}$, and can also be essential for the control of multiple coupled outputs ${ }^{18}$. Here, we first show that a number of buffering topologies can stabilise the original antithetic control system and preserve perfect adaptation. We then show that buffering can allow in- 
creased feedback strength or 'gain' without producing oscillations, which in turn reduces the steady state error even in fast growth conditions. To illustrate the utility of this new antithetic control strategy, we examine two case studies that involve the control of biofuel production and growth rate in microbes.

\section{BACKGROUND}

\section{A. Perfect adaptation and antithetic control}

Antithetic control employs a feedback mechanism with two molecular components that sequester and annihilate each other (see Figure 1B). In its most basic formulation, an antithetic system contains a two-species molecular process to be controlled, and a two-species antithetic controller. The two species of the controlled process $\left(x_{1}\right.$ and $\left.x_{2}\right)$ can represent a variety of molecular systems, including e.g. mRNA and protein as in Figure 1A. The goal of the antithetic control system is to desensitise the steady state concentration of $x_{2}$ with respect to external perturbations. Such perturbations include, for example, insults of molecular species coming from upstream or downstream processes, changes in cellular growth conditions, or alterations to binding affinities between species.

In the absence of stochastic effects, the feedback system can be modelled by the ODEs:

$$
\begin{aligned}
& \dot{x}_{1}=\theta_{1} z_{1}-\gamma_{p} x_{1}, \\
& \dot{x}_{2}=k x_{1}-\gamma_{p} x_{2}, \\
& \dot{z}_{1}=\mu-\eta z_{1} z_{2}-\gamma_{c} z_{1}, \\
& \dot{z}_{2}=\theta_{2} x_{2}-\eta z_{1} z_{2}-\gamma_{c} z_{2},
\end{aligned}
$$

where $z_{1}$ and $z_{2}$ are the concentrations of species in the antithetic controller, and $\theta_{1}, k$, and $\theta_{2}$ are positive parameters representing first-order kinetic rate constants. The parameter $\mu$ describes a zero-order influx of controller species $z_{1}$, while $\eta$ is a second-order kinetic rate constant. We further assume that molecular species are diluted by cellular growth, degraded by other molecular components, or consumed by downstream cellular processes, all of which we model as a first-order clearance with rate constant $\gamma_{p}$. The controller species $z_{1}$ and $z_{2}$, on the other hand, are assumed to be diluted by cellular growth with a rate constant $\gamma_{c}$.

In the absence of dilution effects $\left(\gamma_{c}=0\right)$, from the model in (1) we can write:

$$
\dot{z}_{1}-\dot{z}_{2}=\mu-\theta_{2} x_{2}
$$

which after integration becomes

$$
z_{1}(t)-z_{2}(t)=\theta_{2} \int_{0}^{t}\left(\frac{\mu}{\theta_{2}}-x_{2}\left(t^{\prime}\right)\right) d t^{\prime} .
$$

The above equation means that, if the system has a stable equilibrium, the steady state concentration of $x_{2}$ is
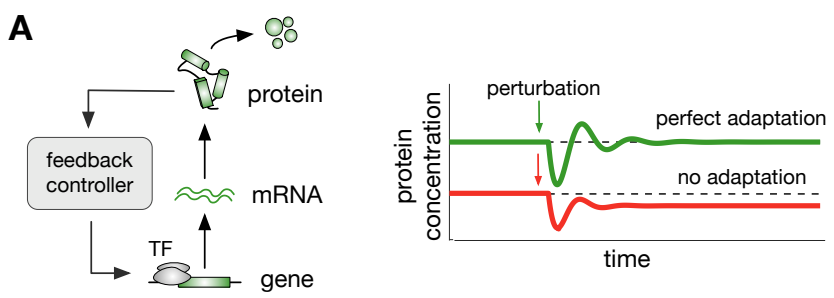

B
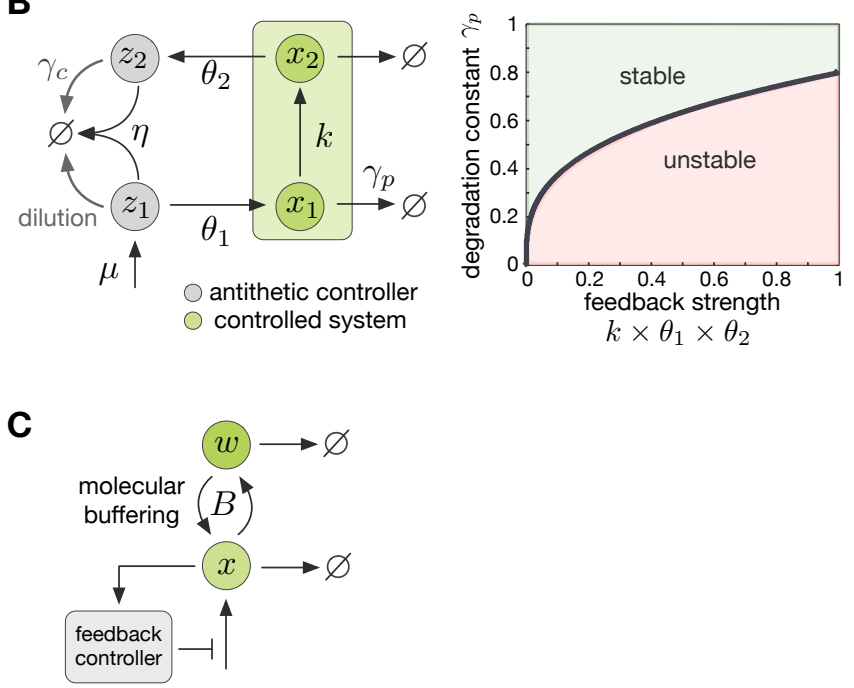

FIG. 1. Perfect adaptation and feedback control. (A) Schematic of a feedback system designed to achieve perfect adaptation in protein expression. Based on readouts of protein concentration, the controller modifies the activity of a transcription factor (TF). If the controller achieves perfect adaptation, steady state protein concentrations are robust to perturbations. (B) Left: the antithetic feedback controller, first proposed Briat et al $\mathrm{in}^{4}$, can achieve perfect adaptation. In the presence of dilution $\left(\gamma_{c} \neq 0\right)$, the antithetic controller does not achieve perfect adaptation. Right: conditions for stability in the case of a two-species system. The stability boundary is the condition in (2); the example was computed with fixed parameters $k=\theta_{2}=\gamma_{p}=1$. (C) In the example, molecular buffering provides a general mechanism to stabilize feedback control systems ${ }^{17}$. The buffer reversibly sequesters molecules of species $x$ into an inactive form $w$.

$x_{2}=\mu / \theta_{2}$, and hence independent of all model parameters except $\mu$ and $\theta_{2}$. Therefore the antithetic control system displays perfect adaptation because the steady state of $x_{2}$ is robust to perturbations in parameters $k, \theta_{1}$, $\theta_{2}$ and $\eta$.

A caveat of antithetic feedback is that it can have a destabilising effect. When the controller species are not diluted $\left(\gamma_{c}=0\right)$, it can be shown that a parametric condition for stability is ${ }^{27}$ :

$$
\gamma_{p}^{3}>\frac{k \theta_{1} \theta_{2}}{2} .
$$

As shown by the stability diagram in Figure 1B, strong antithetic feedback can cause the system to lose perfect 
adaptation and display oscillatory dynamics. Moreover, in the presence of dilution of the controller species $\left(\gamma_{c}>\right.$ $0)$, the antithetic controller is unable to produce perfect adaptation ${ }^{27}$. The adaptation error can be reduced with stronger feedback, for example by increasing the rate constants $\theta_{1}, k$, or $\theta_{2}$. Yet as mentioned above, stronger feedback can cause unwanted oscillations ${ }^{27}$. These caveats are particularly relevant in bioproduction applications that require fast culture growth ${ }^{10,35}$.

\section{B. Molecular buffering}

Buffering is the use of molecular reservoirs to maintain the concentration of chemical species ${ }^{17}$. It is a widespread regulatory mechanism found across all domains of life, with common examples including $\mathrm{pH}$, ATP and calcium buffering ${ }^{21,32}$. Molecular buffering can have a number of regulatory roles ${ }^{17,18}$, including acting as a stabilising mechanism for other molecular feedback systems ${ }^{17}$.

To provide a background on buffering models, we consider the simple case of a chemical species $(x)$ that is subject to feedback regulation, as shown in Figure 1C. A general model for such process is:

$$
\begin{aligned}
\dot{x} & =\underbrace{p(x)}_{\begin{array}{c}
\text { production } \\
\text { with feedback }
\end{array}}-\underbrace{\gamma_{x} x}_{\text {removal }}+\underbrace{g_{w}(w)-g_{x}(x)}_{\text {buffering }}, \\
\dot{w} & =\underbrace{g_{x}(x)-g_{w}(w)}_{\text {buffering }}-\underbrace{\gamma_{w} w}_{\text {removal }},
\end{aligned}
$$

where $w$ is a molecular buffer for the regulated species $x$, and $p(x)$ is a feedback-regulated production rate of $x$. The parameters $\gamma_{x}$ and $\gamma_{w}$ are first-order clearance rate constants of $x$ and $w$, respectively. The terms $g_{w}$ and $g_{x}$ describe the reversible binding of species $x$ and the buffer $w$. The steady state $(\bar{x}, \bar{w})$ occurs when production matches degradation (i.e. $p(\bar{x})=\gamma_{x} \bar{x}+\gamma_{w} \bar{w}$ ) and conversion from $x$ to $w$ matches the reverse conversion plus removal (i.e. $\left.g_{w}(\bar{w})=g_{x}(\bar{x})+\gamma_{w} \bar{w}\right)$.

It can be shown that after linearisation and assuming that the buffering reactions rapidly reach quasiequilibrium, the model (3) can be simplified to (see SI1):

$$
(1+B) \Delta \dot{x}=-\underbrace{h \Delta x}_{\text {feedback }}-\underbrace{\left(\gamma_{x}+B \gamma_{w}\right) \Delta x}_{\text {removal }}
$$

where $\Delta x=x-\bar{x}$ is the deviation of $\mathrm{x}$ from the steady state $\bar{x}, h=-\partial p / \partial x$ is the linearised feedback gain and $B$ is the buffer equilibrium ratio:

$$
B=\frac{\Delta w}{\Delta x},
$$

where $\Delta w=w-\bar{w}$ is the deviation of $w$ from the steady state $\bar{w}$. The parameter $B$ is buffer-specific and quantifies the change in the concentration of a regulated species $(x)$ to the change in the concentration of a buffering species $(w)$ when the buffering reactions are at quasiequilibrium ${ }^{17,18}$.

From (4) we observe that buffering slows down the rate of change of the output $x$ by a factor of $(1+B)$. It can be shown ${ }^{17}$ that this slowed rate generally helps to attenuate fast disturbances and stabilise unwanted oscillations (see SI1). More generally, the stabilisation effect of buffering results from two properties. First, the buffering reactions counteract changes to a target molecular species by acting directly on the species and not via indirect or complex feedback loops ${ }^{16,17}$. Second, buffering can be shown to be mathematically equivalent to popular feedback strategies known to have useful stabilisation properties ${ }^{2}$. In particular, rapid buffering without degradation $\left(\gamma_{w}=0\right)$ is equivalent to negative derivative feedback ${ }^{17}$ :

$$
\Delta \dot{x}=\underbrace{-B \Delta \dot{x}}_{\begin{array}{c}
\text { derivative } \\
\text { feedback }
\end{array}}-\underbrace{h \Delta x}_{\begin{array}{c}
\text { proportional } \\
\text { feedback }
\end{array}}-\gamma_{x} \Delta x,
$$

where the buffer equilibrium ratio $B$ corresponds to the derivative feedback gain commonly employed in control engineering. Likewise, the general case of non-rapid buffering with degradation is mathematically equivalent to the so-called "lead" controller employed in control engineering ${ }^{16}$. In the next section, we study the ability of buffering to stabilise oscillations in antithetic control systems.

\section{BUFFERING CAN STABILISE ANTITHETIC INTEGRAL FEEDBACK}

In this section we study a modified version of the antithetic feedback controller that includes buffering of its molecular components. We consider a number of architectures (Figure 2A) and identify those that suppress oscillations caused by the instability illustrated in Figure 1B. We show that rapid buffering without degradation does not improve stability, while non-rapid buffering and rapid buffering with degradation are highly effective stabilisers.

\section{A. Rapid Buffering}

To study the impact of buffering on the antithetic controller, we consider mathematical models for the topologies in Figure 2A, in which species $z_{1}, z_{2}$, and $x_{2}$ are buffered by molecules $w_{1}, w_{2}$, and $w_{x}$, respectively. For simplicity, we use linear buffering reaction rates in order to focus on the nonlinearity due to the mutual annihilation of controller species $z_{1}$ and $z_{2}{ }^{9}$. As in Eq. (4), we assume that the buffers rapidly reach quasi-equilibrium 
A
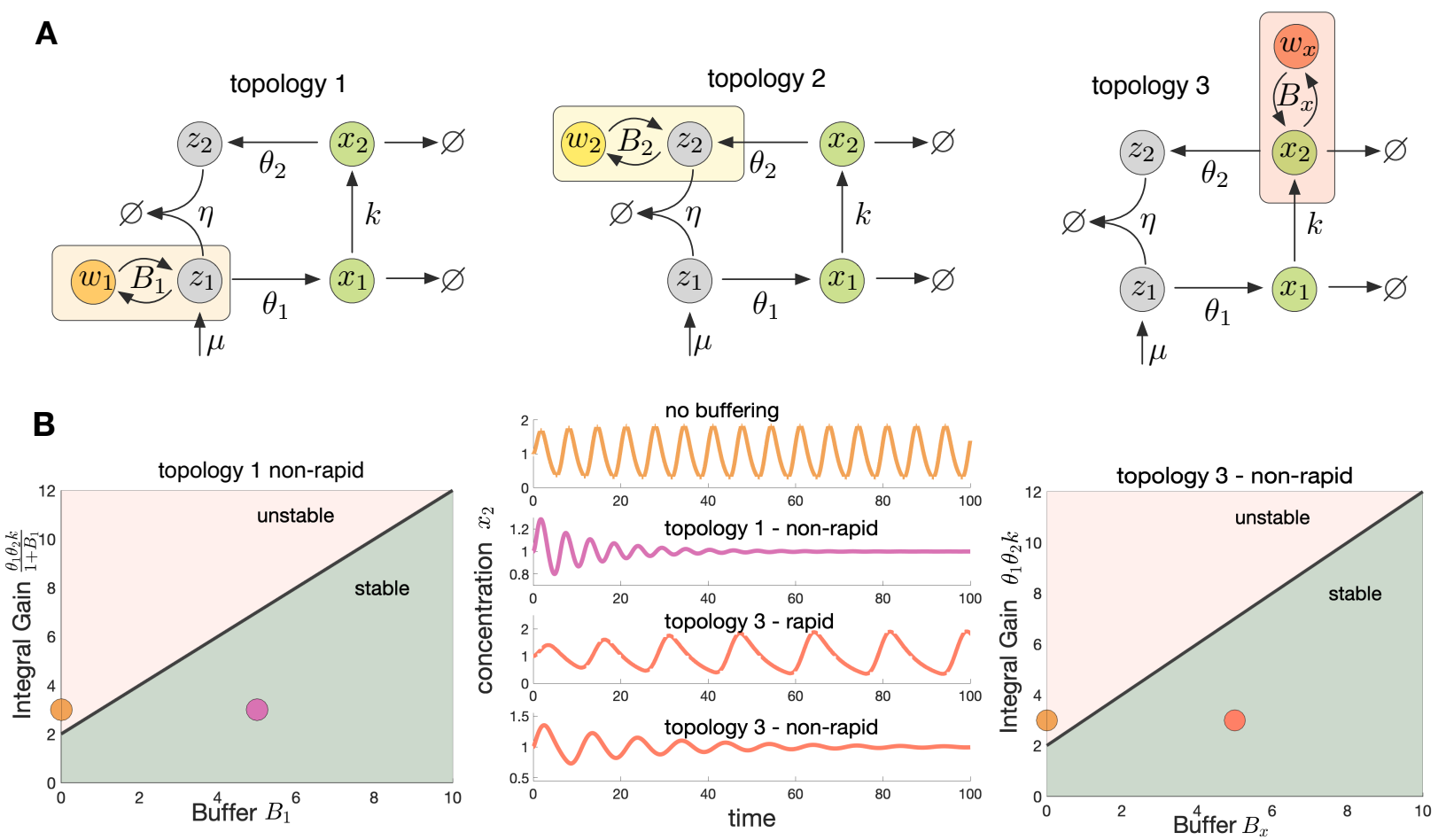

FIG. 2. Buffering stabilizes antithetic feedback . (A) Schematics of three buffered antithetic systems, without dilution $\left(\gamma_{c}=0\right)$; each topology includes buffering a specific molecular species. (B) Stability diagram for buffered topologies 1 and 3 . The stability boundary on the left corresponds to the condition in (10) and the boundary on the right to (11). The time courses show simulations of the output species $\left(x_{2}\right)$ for different topologies. Parameters are $\mu=1, \theta_{1}=3, \theta_{2}=1, \gamma_{p}=1, \eta=100$ and $k=1$. With topology 1 , parameters are $B_{1}=5, \theta_{1}=18$ and $b_{w z}=1$ (non-rapid). With topology 3 , parameters are $B_{x}=5, \theta_{1}=3, b_{w x}=1$ (non-rapid) and $b_{w x}=20$ (rapid).

to obtain the following extended model (see SI2.1):

$$
\begin{aligned}
\dot{x}_{1} & =\theta_{1} z_{1}-\gamma_{p} x_{1} \\
\left(1+B_{x}\right) \dot{x}_{2} & =k x_{1}-\left(\gamma_{p}+B_{x} \gamma_{x}\right) x_{2} \\
\left(1+B_{1}\right) \dot{z}_{1} & =\mu-\eta z_{1} z_{2} \\
\left(1+B_{2}\right) \dot{z}_{2} & =\theta_{2} x_{2}-\eta z_{1} z_{2},
\end{aligned}
$$

where $B_{x}, B_{1}$ and $B_{2}$ are the equilibrium ratios for each buffer, and $\gamma_{x}$ represents the degradation rate of buffer $w_{x}$. The extended model in (6) reduces to the original antithetic system in (1) if $B_{x}=0, B_{1}=0$ and $B_{2}=0$.

In the extended antithetic controller with rapid buffering, the parameters $\left(B_{x}, B_{1}, B_{2}, \gamma_{x}\right)$ are additional tuning knobs that can be used to shape the closed-loop dynamics. In Figure 2A we show the three considered buffering architectures.

We first show that buffered antithetic feedback preserves perfect adaptation. From (6) we write

$$
\left(1+B_{1}\right) \dot{z}_{1}-\left(1+B_{2}\right) \dot{z}_{2}=\mu-\theta_{2} x_{2},
$$

which after integrating becomes:

$$
\begin{aligned}
\left(1+B_{1}\right) z_{1}(t)- & \left(1+B_{2}\right) z_{2}(t) \\
= & \theta_{2} \int_{0}^{t}\left(\frac{\mu}{\theta_{2}}-x_{2}\left(t^{\prime}\right)\right) d t^{\prime} .
\end{aligned}
$$

The above integral ensures that if the system is stable then the steady state of $x_{2}$ is $x_{2}=\mu / \theta_{2}$, hence independent of all parameters except $\mu$ and $\theta_{2}$. The steady state of $x_{2}$ is thus robust to perturbations in the original parameters $k, \theta_{1}, \theta_{2}$, and $\eta$, as well as the additional parameters introduced by the buffering mechanism $B_{x}$, $B_{1}, B_{2}$, and $\gamma_{x}$. This means that the buffered antithetic feedback displays perfect adaptation as in the original formulation in Eq. (1).

Assuming strong integral binding (large $\eta$ ) then $z_{2}$ is small and so with rapid buffering we have the antithetic input in to the controlled system (see Figure 1B and SI4.1)

$$
\theta_{1} z_{1}=\underbrace{\frac{\theta_{1} \theta_{2}}{\left(1+B_{1}\right)}}_{\begin{array}{c}
\text { integral } \\
\text { feedback gain }
\end{array}} \underbrace{\int_{0}^{t}\left(\frac{\mu}{\theta_{2}}-x_{2}\left(t^{\prime}\right)\right) d t^{\prime}}_{\begin{array}{c}
\text { negative } \\
\text { integral feedback }
\end{array}},
$$

where $\theta_{1} z_{1}$ represents the control of $x_{1}$ production in (6) and we can observe that the integral gain is dependent upon $B_{1}$. This changes the gain because rapid buffers tend to slow down the dynamics of $z_{1}$ in (6) and for integral feedback the gain is inversely proportional to the time scale of $z_{1}$.

As shown by the stability condition in (2), the origi- 
A

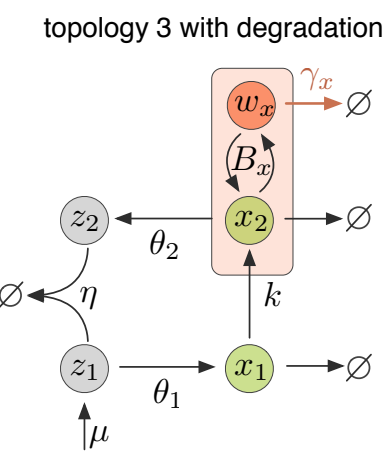

B

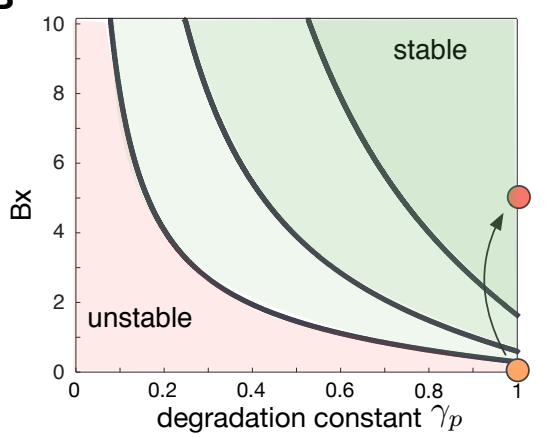

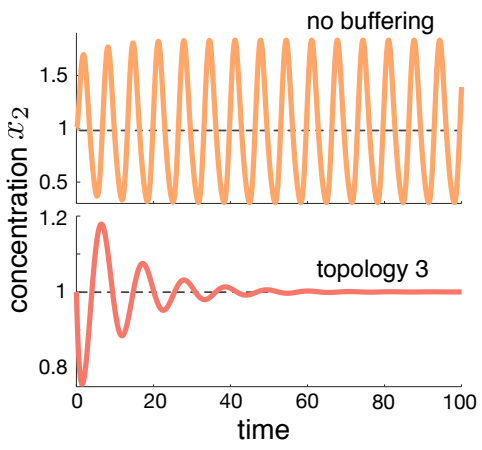

FIG. 3. Stabilizing effect of topology 3 with degradation. (A) We revisit topology 3 with degradation of the buffer with degradation rate constant $\gamma_{x}$. (B) Stability diagram for increasing values of the degradation rate constant $\gamma_{x}=\{0.5,1,2\}$; the stability boundary corresponds to the condition in (12). Time courses are simulations of the output species $\left(x_{2}\right)$ for two representative cases. Parameter values are $\mu=1, \theta_{1}=2, \theta_{2}=1, \gamma_{p}=1, \eta=100$ and $k=1$. For the case of buffering $B_{x}=20$ and $\gamma_{x}=1$.

nal antithetic control system becomes oscillatory when the feedback gain is too strong or the degradation of $x_{1}$ and $x_{2}$ is too slow ${ }^{27}$. To analyse the stabilising role of buffering, we first consider the system in the absence of degradation of the $w_{x}$ buffer (i. e. $\gamma_{x}=0$ ). Assuming rapid buffering and strong integral binding (large $\eta$ ), we find that the system is stable when (see SI2.1):

$$
\gamma_{p}^{3}>\underbrace{\frac{\theta_{1} \theta_{2} k}{\left(1+B_{1}\right)}}_{\begin{array}{c}
\text { integral } \\
\text { feedback } \\
\text { gain }
\end{array}} \underbrace{\frac{1+B_{x}}{2+B_{x}}}_{\begin{array}{c}
\text { stabilisation } \\
\text { effect }
\end{array}} .
$$

From the condition (8) we observe that increasing $B_{1}$ reduces the lower bound for $\gamma_{p}$ and improves stability. However, from (7) we also observe that such effect is equivalent to a reduction in the feedback gain by a factor $\left(1+B_{1}\right)$. Therefore a similar effect can be obtained simply by changing the gain $\theta_{1} \theta_{2}$ without the additional complexity of buffering $z_{1}$. Moreover, the condition in (8) also shows that rapid buffering of $z_{2}$ has no impact on stability, because the stability boundary is independent of $B_{2}$, whereas rapid buffering of $x_{2}$ can destabilise the system and produce oscillations, because the stability boundary becomes more stringent for large $B_{x}$.

\section{B. Non-Rapid Buffering in Topologies 1 and 3}

We next sought to determine the stabilisation properties of non-rapid buffering, i. e. implemented with buffers that do not satisfy the rapid equilibrium assumption. We show that non-rapid buffering can be a highly effective stabilisation strategy for Topologies 1 and 3 , and this stabilisation effect is greater than the effect attributed to the change in feedback gain alone.

To model the effect of non-rapid buffering, we replace the model for $\dot{x}_{2}$ and $\dot{z}_{1}$ in (6) by

$$
\begin{aligned}
\dot{x}_{2} & =k x_{1}-\gamma_{p} x_{2}-b_{x} x_{2}+b_{w x} w_{x} \\
\dot{w}_{x} & =b_{x} x_{2}-b_{w x} w_{x} \\
\dot{z}_{1} & =\mu-\eta z_{1} z_{2}-b_{z} z_{1}+b_{w z} w_{z} \\
\dot{w}_{z} & =b_{z} z_{1}-b_{w z} w_{z},
\end{aligned}
$$

and keep the models for $\dot{x}_{1}$ and $\dot{z}_{2}$ identical as in (6). Given that in the previous section we found that topology 2 does not alter stability, from here onward we set $B_{2}=0$. For topologies 1 and 3 , the buffer equilibrium ratios $\left(B_{1}, B_{x}\right)$ determine the concentration of the buffering species at equilibrium and the reverse reaction rate constants $\left(b_{w z}, b_{w x}\right)$ determine the speed at which the buffers reach equilibrium. These four parameters can be regarded as tuning knobs for modifying the closedloop dynamics, akin to the role of $B_{1}$ and $B_{x}$ in the rapid buffering case of the previous section.

For Topology 1 (i.e. $b_{x}=b_{w x}=0$ ) and assuming strong antithetic binding (large $\eta$ ), the stability condition is:

$$
\gamma_{p}^{3}>\underbrace{\frac{\theta_{1} \theta_{2} k}{\left(1+B_{1}\right)}}_{\begin{array}{c}
\text { integral } \\
\text { feedback } \\
\text { gain }
\end{array}} \underbrace{\frac{1}{\left(2+B_{1}\right)}}_{\begin{array}{c}
\text { stabilisation } \\
\text { effect }
\end{array}}
$$

whereas for Topology 3 (i.e. $b_{z}=b_{w z}=0$ ) the stability condition becomes:

$$
\gamma_{p}^{3}>\frac{\theta_{1} \theta_{2} k}{2+B_{x}}
$$

To derive both conditions (10)-(11) we have assumed for simplicity that $b_{w z}=\gamma_{p}$ and $b_{w x}=\gamma_{p}$, respectively. The general stability condition for other values of $b_{w z}$ and $b_{w x}$ can be found in SI2.2-2.3.

As shown by the stability diagrams in Figure 2, the conditions in (10)-(11) reveal that increases to the buffering ratios $B_{1}$ and $B_{x}$ relax the upper bound for integral 
feedback gain and thus improve stability; this suggests that non-rapid buffering of both $z_{1}$ and $x_{2}$ provide an effective route to suppress oscillations. As shown in SI2.22.3, this stabilisation effect is stronger for $b_{w z}>\gamma_{p}$ in Topology 1 and $b_{w x}<\gamma_{p}$ in Topology 3 .

\section{Rapid Buffering with Degradation}

So far we have assumed that the buffers are not subject to degradation. To establish the impact of buffer degradation on stability, here we show that buffer degradation in Topology 3 can suppress oscillations and preserve perfect adaptation; this new topology is shown in Figure 3A. Under the same assumptions as condition (8) (rapid buffering and strong binding rate constant $\eta$ ), the stability conditions are (see SI2.1):

$$
\gamma_{p}^{3}>\frac{\theta_{1} \theta_{2} k}{2+\left(1+\frac{\gamma_{x}}{\gamma_{p}}\right) B_{x}} \frac{1+B_{x}}{1+\frac{\gamma_{x}}{\gamma_{p}} B_{x}} .
$$

Condition (12) reduces to the one in (8) if $\gamma_{x}=0$ and there is no buffering of $z_{1}$, i.e. $B_{1}=0$.

As shown by the stability diagram in Figure 3B, topology 3 with degradation provides an effective solution to stabilise the closed-loop. The condition in (12) suggests that the ratio $\gamma_{x} / \gamma_{p}$ has a key role in stability. Buffers with shorter half-lives (larger $\gamma_{x}$ ) tend to almost completely remove the instability, even for low buffer equilibrium ratios $B_{x}$. As we show in SI2.4, under the condition $\gamma_{x} / \gamma_{p}>1 / 3$, increases in $B_{x}$ tend to stabilise the closed-loop. This includes the important case where both $x_{2}$ and its buffer are degraded at the same rate, i.e. $\gamma_{x}=\gamma_{p}$. For large values of $B_{x}$, the stabilisation effect is even stronger and becomes independent of the half-life of $w_{x}$. We found a similar stabilisation effect in systems with $x_{1}$ buffering that include degradation of the buffer (see SI2.5).

\section{ACHIEVING NEAR PERFECT ADAPTATION IN FAST GROWTH}

It is well-known that dilution by cell growth can disrupt perfect adaptation in the original antithetic control system $^{1}$. Thus here we explore the impact of dilution in the proposed topologies with molecular buffering. To study the effect of dilution, we modify (9) to include dilution terms for the control species $z_{1}$ and $z_{2}$, as well as the buffer for $z_{1}$ :

$$
\begin{aligned}
\dot{x}_{1} & =\theta_{1} z_{1}-\gamma_{p} x_{1} \\
\dot{x}_{2} & =k x_{1}-\gamma_{p} x_{2}-b_{x} x_{2}+b_{w x} w_{x} \\
\dot{w}_{x} & =b_{x} x_{2}-b_{w x} w_{x}-\gamma_{x} w_{x} \\
\dot{z}_{1} & =\mu-\eta z_{1} z_{2}-\gamma_{c} z_{1}-b_{z} z_{1}+b_{w z} w_{z} \\
\dot{w}_{z} & =b_{z} z_{1}-b_{w z} w_{z}-\gamma_{c} w_{z} \\
\dot{z}_{2} & =\theta_{2} x_{2}-\eta z_{1} z_{2}-\gamma_{c} z_{2} .
\end{aligned}
$$

where $\gamma_{c}$ represents the dilution rate constant of the control species $z_{1}, z_{2}$ and buffer species at $z_{1}$. We assume that dilution of $x_{1}$ and $x_{2}$ and the buffer at $x_{2}$ can be lumped into their first-order degradation rates. As in the previous section, the model (13) can be further simplified for the rapid buffering case (see SI3.1).

\section{A. Topology 3 with dilution}

We found that buffering at $x_{2}$ can reduce steady state error by enabling an increase to the feedback gain without causing oscillations. For the case of dilution with a single buffer at $x_{2}$, we set $b_{z}=b_{w z}=0$ in (13). The resulting steady state is (see SI3.1):

$$
x_{2}=\left(\frac{\mu}{\theta_{2}}\right) \times\left(\frac{1}{1+\Omega_{x}^{-1}}\right),
$$

where

$$
\Omega_{x}=\frac{\alpha}{\gamma_{c}\left(1+B_{x} \frac{\gamma_{x}}{\gamma_{p}}\right)}
$$

and $\alpha=\theta_{1} \theta_{2} k / \gamma_{p}^{2}$. The second term on the right hand side of (14) is always smaller than unity. Therefore the steady state of the output is $x_{2}<\mu / \theta_{2}$ and the system loses perfect adaptation. Moreover, the deviation of the steady state of $x_{2}$ from the reference point $\mu / \theta_{2}$ is (see SI3.1):

$$
\frac{x_{2 n}-x_{2}}{x_{2 n}}=\frac{1}{1+\Omega_{x}}
$$

where $x_{2 n}=\mu / \theta_{2}$ is the reference input. Increases to $B_{x}, \gamma_{c}$ or $\gamma_{x}$ in (16) thus amplify the steady state error, while increases to the feedback strength $k \theta_{1} \theta_{2}$ brings the system closer to perfect adaptation.

We first obtained conditions for stability in Topology 3 with dilution and non-rapid buffering (see SI3.2). Setting $\gamma_{x}=\gamma_{c}$ in (13) we get:

$$
\Omega_{x}<\frac{\gamma_{p}}{\gamma_{c}} \frac{2+B_{x}}{1+B_{x} \frac{\gamma_{c}}{\gamma_{p}}}\left(\left(1+\frac{\gamma_{c}}{\gamma_{p}}\right)^{2}+2 B_{x} \frac{\gamma_{c}}{\gamma_{p}}\right),
$$

where for simplicity we have set $b_{w x}=\gamma_{p}-\gamma_{c}$. The stability conditions for general choices of $b_{w}$ can be found in SI3.2.

Taken together, the relations in (16)-(17) define an upper bound for the best possible steady state error. Specifically, in (16) we see that stronger feedback gain can increase $\Omega_{x}$ and so reduce the steady state error. Buffering of $x_{2}$ tends to stabilise the oscillations and, at the same time, allows the steady state error to be reduced by stronger feedback gain, without the risk of instability observed in the original antithetic mechanism. This phenomenon is illustrated in Figure 4A, which shows the stability condition (17). 
A

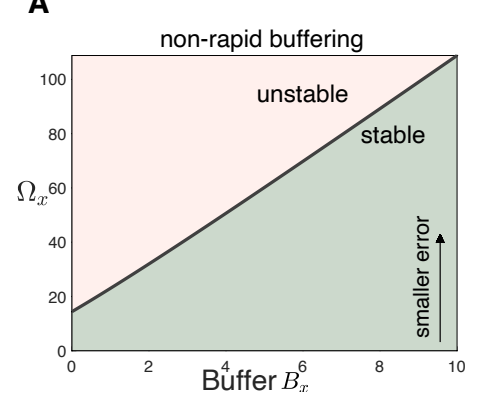

B

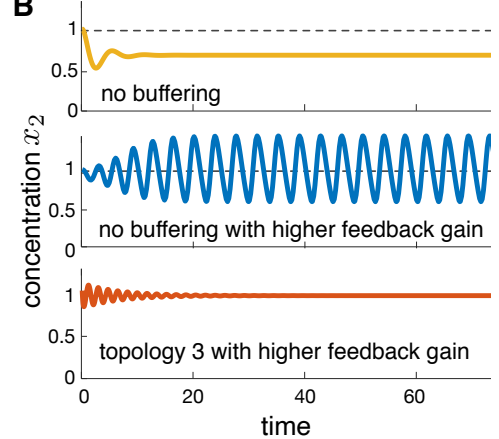

C

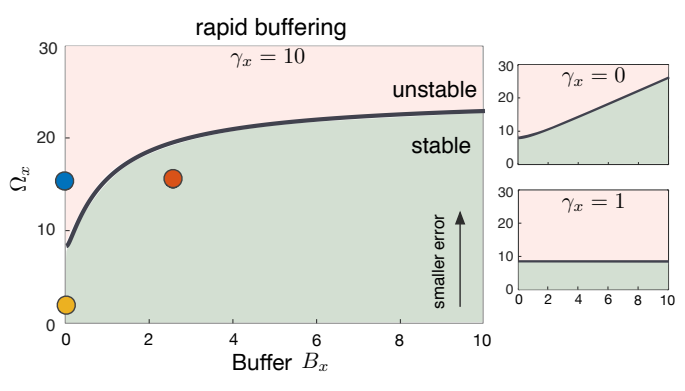

FIG. 4. Adaptation in Topology 3 with dilution. (A) Stability condition in (17) for non-rapid buffering with rate constants $\gamma_{c} / \gamma_{p}=0.2$. (B) Simulation with varying feedback gain $\theta_{1}=\{2,15,400\}$ (from top) and buffer constant $\left(B_{x}=\{0,2.5\}\right.$ ). In all simulations the model parameters are $k=1, \theta_{2}=1$, and $\gamma_{p}=1, \gamma_{c}=1$ and $\gamma_{x}=10$. (C) Stability condition in (18) for rapid buffering with rate constants $\gamma_{c}=1$ and $\gamma_{p}=1$.

In the case of rapid buffering, the condition for stability becomes (see SI3.1):

$$
\begin{aligned}
& \Omega_{x}<\left(1+\frac{\gamma_{c}}{\gamma_{p}}\right)(1+A)\left(\frac{\gamma_{p}}{\gamma_{c}}+A^{-1}\right) \\
& A=\frac{1+B_{x} \frac{\gamma_{x}}{\gamma_{p}}}{1+B_{x}} .
\end{aligned}
$$

As in the non-rapid case, the relations in (16) and (18) define an upper bound for the best possible steady state error. This phenomenon is illustrated in Figure $4 \mathrm{C}$, which shows the stability condition (18). Notably, we observe that increasing $B_{x}$ improves stability only in regions for low and high values of $\gamma_{x}$, and not intermediate values. Figure $4 \mathrm{~B}$ shows simulations of the stabilising effect of molecular buffering for the case of $\gamma_{x}=10$, which enables a decrease of steady state error by means of stronger feedback gain. We also found that topology 3 improves stability even without buffer degradation $\left(\gamma_{x}=0\right)$, which can be observed in Figure $4 \mathrm{~B}$. This improvement differs from the case when there is no dilution in (8).

\section{B. Topology 1 with dilution}

We found that non-rapid buffering at $z_{1}$ can similarly reduce steady state error via increases to the feedback gain. To examine this result in detail, we set $B_{x}=0$ in (13) and compute the resulting steady state (see SI3.4):

$$
x_{2}=\left(\frac{\mu}{\theta_{2}}\right) \times\left(\frac{1}{1+\Omega_{1}^{-1}}\right)
$$

where

$$
\Omega_{1}=\frac{\alpha}{\gamma_{c}\left(1+B_{1}\right)}
$$

and $\alpha=\theta_{1} \theta_{2} k / \gamma_{p}^{2}$. As in the previous case, the steady state satisfies $x_{2}<\mu / \theta_{2}$ and thus the system loses per- fect adaptation (see Figure 5). Moreover, in this case the steady state error is (see SI3.4):

$$
\frac{x_{2 n}-x_{2}}{x_{2 n}}=\frac{1}{1+\Omega_{1}}
$$

where $x_{2 n}=\mu / \theta_{2}$ is the reference input. Increasing $B_{1}$ or $\gamma_{c}$ in (21) increases the steady state error, while increasing the feedback strength $k \theta_{1} \theta_{2}$ brings the system closer to perfect adaptation. If for simplicity we assume that $b_{w z}=\gamma_{p}-\gamma_{c}$, the condition for stability is (see SI3.5):

$$
\Omega_{1}<\frac{\gamma_{p}}{\gamma_{c}} \frac{\left(2+B_{1}\right)}{\left(1+B_{1}\right)}\left(1+B_{1}+\frac{\gamma_{c}}{\gamma_{p}}\right)\left(1+\frac{\gamma_{c}}{\gamma_{p}}\right) .
$$

The general case for other choices of $b_{w}$ can be found in SI3.5. Non-rapid buffering thus enables a reduction of the steady state error via increased feedback gain without unwanted oscillations. This phenomenon is illustrated in Figure 5A, which shows simulations and the stability condition (22) that describes the upper bound on the steady state error. In contrast, rapid buffering does not improve the stability condition and so does not enable a decrease in the adaptation error (see SI3.4); we have illustrated this phenomenon in Figure 5B via numerical simulations.

While the benefit of buffering described here are for a simple network and a steady state error (adaptation) tradeoff, control theory can be use to mathematically quantify this benefit for more general networks and more general tradeoffs involving both steady state and temporal dynamics (see SI4.3).

\section{CASE STUDIES}

\section{A. Model for biofuel production}

To illustrate the potential of the proposed control topologies, here we employ an existing model for biofuel production that incorporates antithetic $\operatorname{control}^{6}$ (see 
A

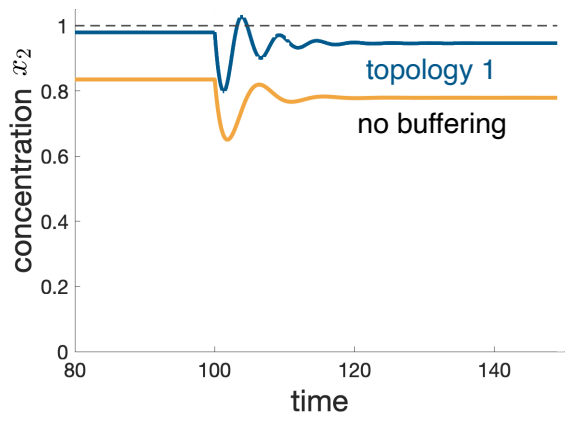

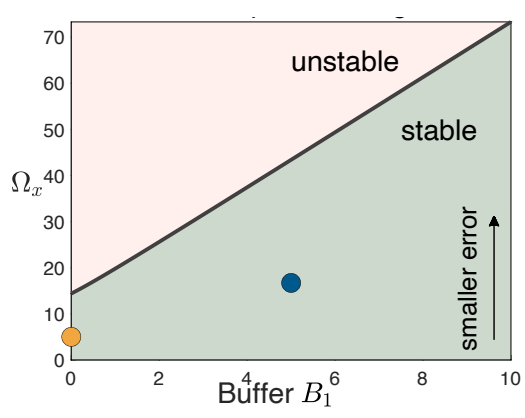

B

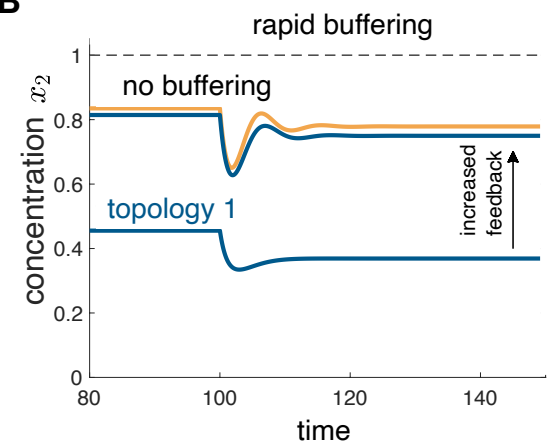

FIG. 5. Adaptation in Topology 1 with dilution. (A) Non-rapid buffering with stronger feedback reduces the adaptation error. (Left) In simulations the model parameters are $\theta_{2}=1, \gamma_{p}=1, \eta=100, \mu=1, \gamma_{c}=0.2$, and $k=1$ changes to $k=0.7$ at $t=100$. (Right) The stability condition in (22) for non-rapid buffering with dilution rate constant in the ratio $\gamma_{c} / \gamma_{p}=0.2$. (B) Rapid buffering increases the adaptation error while rapid buffering with stronger feedback is identical to no buffering with weaker feedback. Parameter values for the non-rapid case are $\theta_{1}=\{1,15\}, B_{1}=\{0,5\}$ and $b_{w 1}=\{0,0.8\}$; parameters for the rapid case are $\theta_{1}=\{1,5\}$ and $B_{1}=\{0,5\}$.

also $^{12}$ ), shown in Figure 6A. The synthetic system produces biofuel from sugars through a metabolic pathway. The biofuel product can be toxic to the cell and so efflux pump proteins are expressed to remove the toxic metabolic product. However, at large concentrations the efflux protein pump can also be toxic. A feedback mechanism can help robustly regulate these two competing toxic products. The antithetic feedback mechanism senses the biofuel concentration to control the expression of efflux pump protein. An increase in the pump protein then reduces the biofuel concentration, completing the loop. Stability is known to be a major issue for the system, as it susceptible to oscillation for large $\eta$, which is the typical design case ${ }^{6}$.

The extended model of the biofuel circuit with antithetic feedback and the addition of a protein buffer is:

$$
\begin{aligned}
\dot{n} & =\alpha_{n} n(1-n)-\delta_{n} b_{i} n-\frac{\alpha_{n} n p}{p+\gamma_{p}} \\
\dot{b}_{i} & =\alpha_{b} n-\delta_{b} p b_{i} \\
\dot{p} & =k z_{2}-\beta_{p} p \\
\dot{b}_{e} & =V \delta_{b} p b_{i} n \\
\dot{z}_{1} & =\mu-\eta z_{1} z_{2} \\
\dot{z_{2}} & =\theta b_{i}-\eta z_{1} z_{2}-I r_{p} p+r_{w} w \\
\dot{w} & =I r_{p} z_{2}-r_{w} w
\end{aligned}
$$

where $n$ is the normalized cell density, which is assumed to follow logistic growth with additional death rates due to toxicity of intracellular biofuel concentration $b_{i}$ and efflux protein pump $p$. The variables $z_{1}$ and $z_{2}$ are the controller species, while the production of the protein pump $p$ is assumed to be proportional to controller species $z_{2}$. The variable $w$ is the buffering species which buffers $z_{2}$ through a reversible reaction via chemical species $I$ that inhibits $z_{2}$ sequestering when bound to $z_{2}$. The variable $b_{e}$ is the extracellular concentration of biofuel.

Buffering of $z_{2}$ can be seen to stabilise the process in the simulations of the model (see Figure 6A). These simulations show that the oscillations, which occur when $\eta$ is too large, quickly settle to the steady state when buffering is introduced. This stabilising effect is equivalent to the impact of buffering in Topology 1 in Section III. Buffering removes the stability limit on $\eta$ and so a large antithetic binding rate $\eta$ is possible without oscillations. This example thus illustrates that the stabilising effect of buffering also occurs in more complex systems than for the simple case presented above.

\section{B. Model for growth control}

For the synthetic growth control case study, we use an existing model of the synthetic growth control circuit, which includes the new addition of buffering 27 (see Figure 6B). The variable $N$ represents the population size and is assumed to follow logistic growth, with an additional death rate due to toxicity that is proportional to the concentration of $C c d B$ per cell. $C c d b$ is a protein that is toxic to the cell. $m R N A$ is messenger RNA while asRNA is a short antisense RNA that has a complementary sequence to the mRNA, which enables sequestration between the two. $m R N A$ and asRNA form the antithetic integral controller. The transcription of mRNA is induced by a quorum-sensing ligand. The term $G_{a}$ represents the gain between $N$ and mRNA induction resulting from the quorum-sensing molecule AHL. $W$ represents a buffer of Ccdb, which consists of an inactivated form of $\mathrm{Ccdb}$ that can reversibly bind to an inhibitor molecule $I$. The adapted model of the genetic 
A
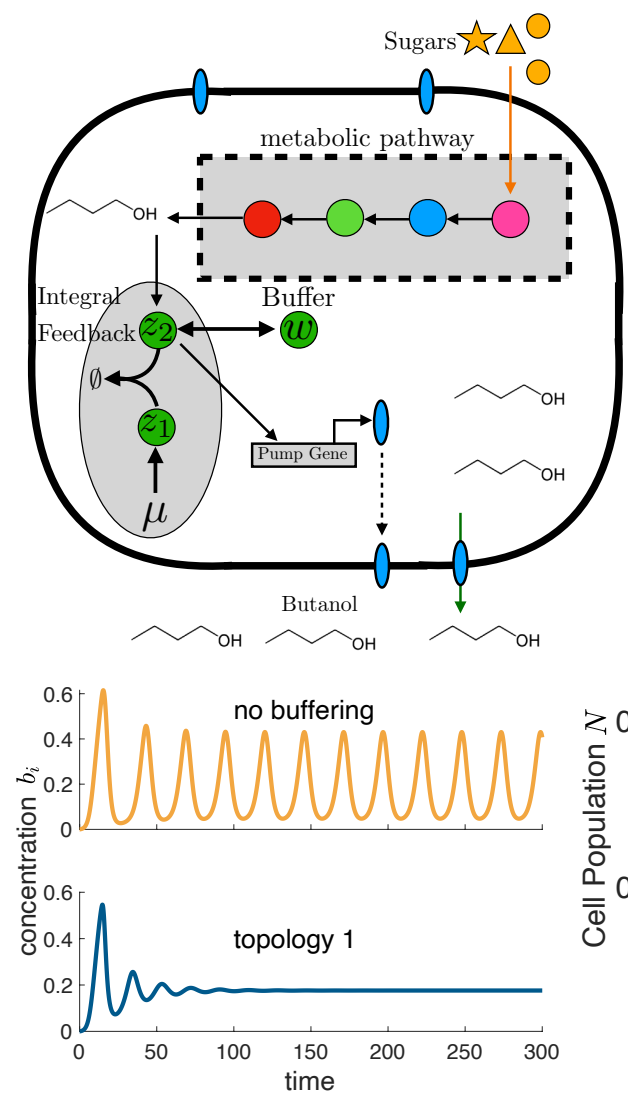

B

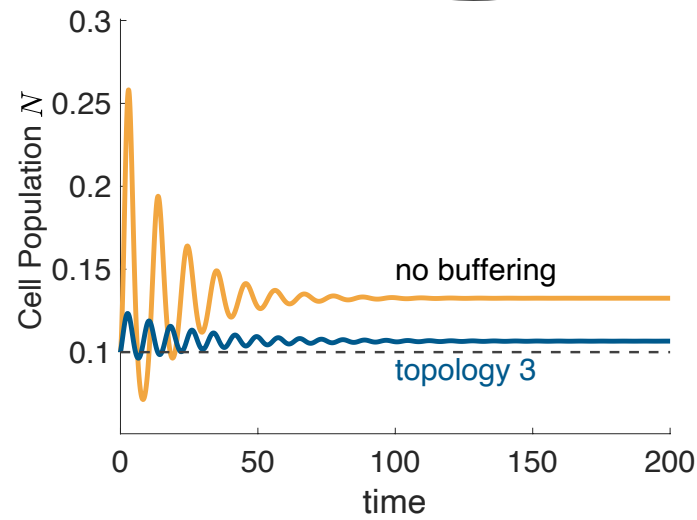

FIG. 6. Case studies of molecular buffering coupled with antithetic control showing improved stabilisation and adaptation. (A) Biofuel production system adapted from ${ }^{6}$ to include buffering of species $z_{2}$. Simulations show the stabilising effect of buffering. Model parameters for simulations are $\alpha_{n}=0.66, \delta_{n}=0.5, \gamma_{p}=0.14, \alpha_{b}=0.1, \delta_{b}=0.5, \beta_{p}=0.66, V=1, \eta=100, \mu=0.1762$, $\theta=1, I=1, r_{p}=0,1.25, r_{w}=0,0.25, k=0.5,3$. $k$ is increased with buffering to compensate for the reduced integral feedback gain from buffering (B) Synthetic growth control circuit ${ }^{27}$ adapted to include buffering of CcdB. Simulations show the ability of buffering to decrease the steady state error via stronger feedback without oscillations. Model parameters for simulations with and without buffering are $\gamma_{p}=3, r=1, \tau=4 \times 10^{-3}, k_{R}=10^{-1}, G=10^{-6}, \eta=20, \gamma_{r}=0.1, \mu=10, N_{m}=10^{9}, \gamma_{w}=10^{-2}$, $k_{p}=\{20,200\}, b_{c}=\{0,7\}$ and $b_{w}=\left\{0,7 \times 10^{-2}\right\}$.

circuit with the new addition of a protein buffer is

$$
\begin{aligned}
\frac{d}{d t}[C c d B] & =k_{p}[m R N A]-\left(\gamma_{p}+b_{C} I\right)[C c d B]+b_{w}[W] \\
\frac{d}{d t}[m R N A] & =k_{R} G_{a} N-\left(\eta[a s R N A]-\gamma_{R}\right)[m R N A] \\
\frac{d}{d t}[a s R N A] & =\mu-\eta[a s R N A][m R N A]-\gamma_{R}[\operatorname{as} R N A] \\
\frac{d}{d t} N & =r N\left(1-\frac{N}{N_{m}}\right)-\tau[C c d B] N \\
\frac{d}{d t}[W] & =b_{C} I[C c d B]-b_{w}[W]-\gamma_{w}[W]
\end{aligned}
$$

where $[\cdot]$ represents intracellular concentrations for each species and the last line indicating the rate of change of $W$ is new to the model.

The buffering of $C c d B$ as shown above is equivalent to $x_{1}$ buffering in the model (6) and Figure 2, as $N$ is the output and equivalent to $x_{2}$. Buffering at $x_{1}$ provides a similar benefit as buffering at $x_{2}$ and so can also enable near-perfect adaptation.

Buffering of $C c d B$ in conjuction with increased feedback gain can be shown to reduce steady state error in the simulations in Figure 6. Increased feedback gain is implemented in these simulations by increasing the translation rate of $C c d B$.

\section{DISCUSSION}

Perfect adaptation has been subject of intense study in the synthetic biology community. Although perfectly adapting systems are ubiquituous in nature, their im- 
plementation has proven particularly elusive. The antithetic control motif, first discovered by Briat et $\mathrm{al}^{4}$ and implemented by Aoki et $\mathrm{al}^{1}$, provides a new molecular mechanism to build perfect adaptation into a wide range of synthetic gene circuits. A number of works have sought to find alternative circuits that provide adaptation properties similar to antithetic control. For example, several authors have shown that ultrasensitive feedback can display some of the features of perfect adaptation $^{25,28}$, and the idea was recently extended in great detail for synthetic gene circuits ${ }^{30}$. Other works have sought to devise molecular implementations of Proportional-Integral-Derivative control $^{9}$, as this is a widely adopted strategy for perfect adaptation in engineered control systems.

Here we have addressed caveats of the original antithetic control system with an extended architecture that has improved stability properties. The proposed circuit combines an antithetic motif with a molecular buffering mechanism. Molecular buffering is widely conserved in natural systems, and common examples include the ATP buffering by creatine phosphate, $\mathrm{pH}$ buffering and calcium buffering. In all these examples, a molecular buffer sequesters a target molecule into an inactive form, resulting in a system with improved ability to mitigate fast perturbations. In the case of antithetic control, the addition of buffering results in the stabilisation of unwanted oscillations and, moreover, provides near-perfect adaptation even in rapid growth conditions where the performance of antithetic control is known to be particularly poor.

After detailed examination of mathematical models for various circuit architectures (Fig. 2) with rapid and non-rapid buffers, we found two candidate systems with improved stability properties, either by buffering species of the antithetic motif itself, or by buffering a target species to be controlled. The first circuit, called Topology 1 in Fig. 2, under non-rapid buffering provides stability over a larger range of parameters values than classic antithetic control and can generally stabilise unwanted oscillations. Moreover, Topology 1 requires buffering of a molecular species of the antithetic motif itself, and therefore it provides a promising strategy to stabilise variables that are not easily buffered directly, such as population size or metabolite species as illustrated by the example in Fig 6A.

The second circuit, termed Topology 3 in Fig. 2, requires buffering the molecular output of the process to be controlled. We found that when $x_{2}$ buffering is either non-rapid or rapid and coupled with degradation, it mitigates oscillations in fast growth regimes. Interestingly, there is a similar effect when applied to intermediate species instead of the output species in the controlled process, such as $x_{1}$ in the original circuit shown in Fig. 2 or CcdB in the growth control case study in Fig. 6. Buffering an intermediate species also provides additional design flexibility when the output species cannot be easily buffered.
Although our results indicate that both buffering and degradation can act as stabilisers of antithetic control, designs with increased buffering and reduced degradation can provide substantial benefits. Increased degradation requires a higher production rate to achieve a particular steady state concentration. If the degradation mechanism requires expression of heterologous proteins, an increase in their production rate imposes a heavier genetic burden on the host cell ${ }^{8,26}$. Moreover, in applications, protein production rates are subject to upper limits depending on the genetic machinery of the host $^{36}$, and so increasing degradation can also limit the maximum set point concentration. Tuning of degradation can also be difficult to implement given the limited number of degradation tags available.

The effect of buffering on adaptation is strikingly similar to a strategy employed in industrial process control, where buffer tanks are employed to regulate and smooth out the impact of disturbances ${ }^{13}$. In our case, the specific implementation of the molecular buffers is a subject of future study, as this will largely depend on the type of biomolecular process to be controlled (see ${ }^{18}$ for quantification of different buffering reaction forms). For example, buffers for gene expression may require gene products to be sequestered, which can be achieved through several mechanisms such as reversible protein-protein binding ${ }^{24}$, phosphorylation ${ }^{19}$, small molecule inhibitors ${ }^{11}$, or DNA decoy sites ${ }^{34}$. In metabolism and signalling systems, ubiquitous examples are the interconversion between a target species and a buffer (e. g. reversible catalysis between ATP and creatine phosphate ${ }^{17,32}$ ) or sequestering by dedicated proteins (e. g. $\mathrm{Ca}^{2+}$ or $\mathrm{H}^{+}$ions ${ }^{17,21,32}$ ).

Our main goal in this paper was to show that molecular buffering can improve perfect adaptation in the antithetic control motif. Since buffering is known to stabilise a much wider range of molecular networks ${ }^{16}$, it also has the potential to improve other circuits implementing perfect adaptation, e. g. those that rely on ultrasensitive behaviour ${ }^{30}$. Further, its properties may be used to instead improve transient response or disturbance rejection of a circuit, rather than only adaptation ${ }^{16,17}$. A key future step is the study of design rules for buffering and antithetic feedback. A useful starting point in this direction are the rules from lead-lag and PID controller tuning from control engineering ${ }^{16}$ (see SI4.2). Unlike technological controllers, however, the stabilisation properties of buffering are tied to its synergy with feedback regulation ${ }^{16,17}$ and the location of the buffer in a network is important, which introduces new challenges for controller design.

For simplicity, here we have focused exclusively on deterministic dynamics, but the analysis of stochastic effects emerging from the interplay between molecular buffering and antithetic control are particularly attractive, as it is known that buffering does not amplify stochastic fluctuations ${ }^{17}$ yet some phenomena are 
known to emerge only in the presence of molecular noise $^{4,31,33}$.

As synthetic gene circuits grow in size and complexity, there is a growing need for mechanisms that can enhance their robustness in a range of operational conditions. In the longer term, this will require the availability of a catalogue of gene circuits that can produce perfect adaptation in response to perturbations. In this work we have presented one such architecture, and thus laid theoretical groundwork for the discovery of biomolecular systems with improved functionality.

\section{METHODS}

All mathematical models are based on systems of ordinary differential equations. The stability conditions in Eqs. (8), (12), (18) and (22) were obtained using frequency domain transformations (Laplace and Fourier) of the linearised models, along with detailed examination of the magnitude and phase equations of the resulting characteristic polynomials for the closed-loop systems ${ }^{27}$. Simulations were carried out using standard ODE solvers in MATLAB. All calculations and model descriptions can be found in the Supplementary Material.

\section{AUTHOR CONTRIBUTIONS}

Conceptualization, E.J.H.; Methodology, E.J.H.; Formal Analysis: E.J.H., and D.A.O.; Investigation, E.J.H., and D.A.O.; Writing - Original Draft, E.J.H. ; Writing Review \& Editing, E.J.H., and D.A.O. ; Funding Acquisition, E.J.H.; Supervision, E.J.H., and D.A.O.

\section{ACKNOWLEDGEMENTS}

The authors would like to thank Morgan Kelly for her assistance. EJH was would like to thank the contribution of Judith and David Coffey.

The authors declare that there are no competing interests.

\section{REFERENCES}

${ }^{1}$ S.K. Aoki, G. Lillacci ans A. Gupta, A. Baumschlager, D. Schweingruber, and M. Khammash. A universal biomolecular integral feedback controller for robust perfect adaptation. Nature, 570:533-537, 2019.

${ }^{2}$ K. J. Aström and R. M. Murray. Feedback Systems. Princeton University Press, 2008.

${ }^{3} \mathrm{~N}$. Barkai and S. Leibler. Robustness in simple biochemical networks. Nature, 387:913-917, 1997.

${ }^{4}$ C. Briat, A. Gupta, and M. Khammash. Antithetic integral feedback ensures robust perfect adaptation in noisy biomolecular networks. Cell Syst., 2(1):15-26, 2016.
${ }^{5}$ C. Briat, A. Gupta, and M. Khammash. Antithetic proportionalintegral feedback for reduced variance and improved control performance of stochastic reaction networks. Royal Society Interface, 15, 2018.

${ }^{6} \mathrm{C}$. Briat and M. Khammash. Perfect adaptation and optimal equilibrium productivity in a simple microbial biofuel metabolic pathway using dynamic integral control. ACS Synthetic Biology, 7(2):419-431, 2018.

${ }^{7}$ D. E. Cameron, C.J. Bashor, and J. J. Collins. A brief history of synthetic biology. Nat. Rev. Microbiol., 12(5):381-390, 2014.

${ }^{8}$ F. Ceroni, R. Algar, G. B. Stan, and T. Ellis. Quantifying cellular capacity identifies gene expression designs with reduced burden. $\mathrm{Na}$ ture Methods, 12(5):415-418, apr 2015.

${ }^{9}$ M. Chevalier, M. Gomez-Schiavon, A.H. Ng, and H. El-Samad. Design and analysis of a proportional-integral- derivative controller with biological molecules. Cell Systems, 9:338-353, 2019.

${ }^{10}$ V. Chubukov, A. Mukhopadhyay, C.J. Petzold, J.D. Keasling, and H.G. Martin. Synthetic and systems biology for microbial production of commodity chemicals. npj systems biology and applications, 2(16009), 2016.

${ }^{11} \mathrm{C}$. Denison and T. Kodadek. Small-molecule-based strategies for controlling gene expression. Chemistry \& Biology, 5:129-145, 1998.

${ }^{12}$ M. J. Dunlop, J.D. Keasling, and A. Mukhopadhyay. A model for improving microbial biofuel production usinga synthetic feedback loop. Syst Synth Biol, 4:95=104, 2010.

${ }^{13} \mathrm{~A}$. Faanes and S. Skogestad. Buffer tank design for acceptable control performance. Ind. Eng. Chem. Res., 42(10):2198-2208, 2003.

${ }^{14} \mathrm{~T}$. Friedlander and N. Brenner. Adaptive response by statedependent inactivation. Proc. Natl. Acad. Sci., 106(52):22558-22563, 2009.

${ }^{15} \mathrm{~A}$. Gupta and M. Khammash. An antithetic integral rein controller for bio-molecular networks. IEEE 58th Conference on Decision and Control (CDC), Nice, France, , pages 2808-2813, 2019.

${ }^{16}$ E.J. Hancock and J.Ang. Frequency domain properties and fundamental limits of buffer-feedback regulation in biochemical systems. Automatica, 103:330-336, 2019.

${ }^{17}$ E.J. Hancock, J.Ang, A.Papachristodoulou, and G-B.Stan. The interplay between feedback and buffering in cellular homeostasis. Cell Systems, 5(5):498-508, 2017.

${ }^{18}$ E.J. Hancock, J.R. Krycer, and J. Ang. Metabolic buffer analysis reveals the simultaneous, independent control of atp and adenylate energy ratios. Royal Society Interface, in press, 2021.

${ }^{19}$ H. Harada, B. Becknell, M. Wilm, M. Mann, L.J. Huang, S.S. Taylor, J.D. Scott, and S.J. Korsmeyer. Phosphorylation and inactivation of bad by mitochondria-anchored protein kinase a. Mol Cell, 3(4):41322, 1999.

${ }^{20}$ J.E. Ferrell Jr. Perfect and near-perfect adaptation in cell signaling. Cell Systems, 2:62-67, 2016.

${ }^{21} \mathrm{~J}$. Keener and J. Sneyd. Mathematical Physiology. Springer-Verlag, 1998.

${ }^{22}$ D. Liu, A.A. Mannan, Y. Han, D.A. Oyarzún, and F. Zhang. Dynamic metabolic control: towards precision engineering of metabolism. J Ind Microbiol Biotechnology, 45(7):535-543, 2018.

${ }^{23}$ W. Ma, A. Trusina, H. El-Samad, W.A. Lim, , and C. Tang. Defining network topologies that can achieve biochemical adaptation. Cell, 138(4):760-73, 2009.

${ }^{24} \mathrm{~J}$. De Melo, L. He, and D. Tang. The protein-protein interactionmediated inactivation of pten. Curr Mol Med, 14(1):22-33, 2014.

${ }^{25}$ F. Montefusco, E.O. Akman, S.O. Soyer, and D. G. Bates. Ultrasensitive negative feedback control: A natural approach for the design of synthetic controllers. PLOS One, 11(8), 2016.

${ }^{26}$ E. M. Nikolados, A. Y. Weiße, F. Ceroni, and D. A. Oyarzún. Growth Defects and Loss-of-Function in Synthetic Gene Circuits. ACS Synthetic Biology, 8(6):1231-1240, 2019.

${ }^{27}$ N. Olsman, A-A. Baetica, F. Xiao, Y.P. Leong, R.M. Murray, and J.C. Doyle. Hard limits and performance tradeoffs in a class of antithetic integral feedback networks. Cell Systems, 9, 2019

${ }^{28}$ D. A. Oyarzún and G.-B. Stan. Synthetic gene circuits for metabolic control: design trade-offs and constraints. Journal of the Royal Society, Interface / the Royal Society, 10(78), oct 2013. 
bioRxiv preprint doi: https://doi.org/10.1101/2021.04.18.440372; this version posted September 29, 2021. The copyright holder for this preprint (which was not certified by peer review) is the author/funder, who has granted bioRxiv a license to display the preprint in perpetuity. It is made available under aCC-BY-NC-ND 4.0 International license.

${ }^{29}$ Y. Qian and D. Del Vecchio. Realizing 'integral control' in living cells: how to overcome leaky integration due to dilution? Royal Society Interface, 15, 2018.

${ }^{30}$ C.C. Samaniego and E. Franco. Ultrasensitive molecular controllers for quasi-integral feedback. Cell Systems, In Press, 2021.

${ }^{31}$ M. Samoilov, S. Plyasunov, and A. P. Arkin. Stochastic amplification and signaling in enzymatic futile cycles through noise-induced bistability with oscillations. Proceedings of the National Academy of Sciences of the United States of America, 102(7):2310-5, feb 2005.

${ }^{32}$ L. Sherwood. Human Physiology: From Cells to Systems. Brooks Cole, 2013.

${ }^{33}$ M. K. Tonn, P. Thomas, M. Barahona, and D. A. Oyarzún. Stochastic modelling reveals mechanisms of metabolic heterogeneity. Communications Biology, 2(1):108, dec 2019.

${ }^{34}$ T. Wang, N. Tague, S.A. Whelan, and M.J. Dunlop. Programmable gene regulation for metabolic engineering using decoy transcription factor binding sites. Nucleic Acids Research, 49(2):1163-1172, 2021.

${ }^{35} \mathrm{M}$. Wehrs, D. Tanjore, T. Eng, J. Lievense, T.R. Pray, and A. Mukhopadhyay. Engineering robust production microbes for large-scale cultivation. Trends in Microbiology, 27(6), 2019.

${ }^{36}$ Andrea Y. Weisse, Diego A. Oyarzún, Vincent Danos, and Peter S. Swain. Mechanistic links between cellular trade-offs, gene expression, and growth. Proceedings of the National Academy of Sciences, 112(9):E1038-E1047, feb 2015. 
Supplementary material

\title{
Stabilisation of Antithetic Control via Molecular Buffering
}

\author{
Edward J. Hancock ${ }^{1,2}$, Diego A. Oyarzún 3,4 \\ ${ }^{1}$ School of Mathematics and Statistics, The University of Sydney, NSW, 2006, Australia \\ ${ }^{2}$ Charles Perkins Centre, The University of Sydney, NSW, 2006, Australia \\ ${ }^{3}$ School of Biological Sciences, University of Edinburgh, UK \\ ${ }^{4}$ School of Informatics, University of Edinburgh, UK
}

\section{SI1. BUFFERING BACKGROUND}

In this section, we provide a background on buffering ${ }^{4}$, including methods for analysing models with buffering. We start with the simple model in the main section of a single regulated species that is being buffered, such that

$$
\begin{aligned}
\dot{x} & =\underbrace{p(x)}_{\begin{array}{c}
\text { production } \\
\text { with feedback }
\end{array}}-\underbrace{\gamma_{x} x}_{\text {removal }}+\underbrace{g_{w}(w)-g_{x}(x)}_{\text {buffering }} \\
\dot{w} & =\underbrace{g_{x}(x)-g_{w}(w)}_{\text {buffering }}-\underbrace{\gamma_{w} w}_{\text {removal }}
\end{aligned}
$$

where $x$ is the output species being regulated, $w$ is the buffering species, $p$ is the production rate of $x, \gamma_{x}$ is the removal kinetic rate of $x, g_{w}$ is the forward buffering reaction rate and $g_{x}$ is the reverse buffering reaction rate. Incorporation of feedback is represented by the $x$ dependence of production. The steady state occurs when production matches degradation $\left(p(\bar{x})=\gamma_{x} \bar{x}+\gamma_{w} \bar{w}\right)$ and the buffer is at steady state $\left(g_{w}(\bar{w})=g_{x}(\bar{x})+\gamma_{w} \bar{w}\right)$. To analyse (S1), we reduce the two state model to one state by assuming that the buffering reactions rapidly reach equilibrium (see $\mathrm{e}^{4}$ for more mathematically rigorous derivation). To carry this out, we first linearise (S1), which results in

$$
\begin{aligned}
& \Delta \dot{x}=\underbrace{-h \Delta x}_{\text {feedback }}-\underbrace{\gamma_{x} \Delta x}_{\text {removal }}+\underbrace{b_{w} \Delta w-b_{x} \Delta x}_{\text {buffering }} \\
& \Delta \dot{w}=\underbrace{-b_{w} \Delta w+b_{x} \Delta x}_{\text {buffering }}-\underbrace{\gamma_{w} \Delta w}_{\text {removal }}
\end{aligned}
$$

where $h=-\frac{\partial p}{\partial x}$ is the linearised feedback gain, and $b_{w}=\frac{\partial g_{w}}{\partial w}$ and $b_{x}=\frac{\partial g_{x}}{\partial x}$ are the linearised kinetic rates for the forward and reverse buffering reaction.

We introduce the variable $\Delta x_{T}=\Delta w+\Delta x$ to carry out a quasi-steady state approximation ${ }^{2,4}$, where we have the transformed model

$$
\begin{aligned}
\Delta \dot{x}_{T} & =-h \Delta x-\gamma_{x} \Delta x-\gamma_{w} \Delta w \\
\Delta \dot{w} & =-b_{w} \Delta w+b_{x} \Delta x-\gamma_{w} \Delta w .
\end{aligned}
$$

We assume that the buffering species $\Delta w$ rapidly reaches quasi-steady state due to large $b_{w}$ and thus assume $\Delta \dot{w}=-\left(b_{w}+\gamma_{w}\right) \Delta w+b_{x} \Delta x=0$, where $x_{T}$ is a 'slow' variable as it is not a direct function of the buffering reactions. The quasi-steady state can be rewritten

$$
\Delta w=B \Delta x, \quad B=\frac{b_{x}}{b_{w}+\gamma_{w}} .
$$

From this quasi-steady state, we have $\Delta x_{T}=(1+B) \Delta x$ and $\Delta \dot{x}_{T}=(1+B) \Delta \dot{x}$, resulting in

$$
(1+B) \Delta \dot{x}=-h \Delta x-\left(\gamma_{x}+B \gamma_{w}\right) \Delta x
$$


which is a reduced one state model, where the second state can be determined from $\Delta w=B \Delta x$.

In technology, integral feedback is often paired with proportional and derivative feedback (PID control) ${ }^{1}$. In biology, rapid buffering without degradation is equivalent to negative derivative feedback and rapid buffering with degradation is equivalent to PD feedback with degradation. These equivalences can be observed in ${ }^{4}$

$$
\Delta \dot{x}=\underbrace{-B \Delta \dot{x}-\gamma_{w} B \Delta x}_{\begin{array}{c}
\text { proportional }+ \\
\text { derivative } \\
\text { feedback }
\end{array}}-\underbrace{h \Delta x}_{\begin{array}{c}
\text { proportional } \\
\text { feedback }
\end{array}}-\gamma_{x} \Delta x
$$

where the buffer equilibrium ratio $B$ corresponds to the derivative feedback 'gain'.

To study the effect of buffering on stability, we can also modify the model in (S1) to include a delay of the production feedback term

$$
\begin{aligned}
\dot{x} & =\underbrace{p(x(t-\tau))}_{\begin{array}{c}
\text { production } \\
\text { with feedback }
\end{array}}-\underbrace{\gamma_{x} x}_{\text {removal }}+\underbrace{g_{w}(w)-g_{x}(x)}_{\text {buffering }} \\
\dot{w} & =\underbrace{g_{x}(x)-g_{w}(w)}_{\text {buffering }}-\underbrace{\gamma_{w} w}_{\text {removal }}
\end{aligned}
$$

where $p(x(t-\tau))$ represents the production feedback with a delay of time $\tau$. The reduced model is

$$
(1+B) \Delta \dot{x}=-h \Delta x_{\tau}-\left(\gamma_{x}+B \gamma_{w}\right) \Delta x
$$

where $\Delta x_{\tau}=\Delta x(t-\tau)$.

It can be observed in Figure S2 that buffering can stabilise the oscillations that result from feedback delay. This stabilisation is a result of (a) the buffering reactions acting directly on the target molecular species (i.e. not being subject to delays) and (b) buffering having a 'derivative feedback' effect - known for its stabilising effects in control engineering ${ }^{1}$.

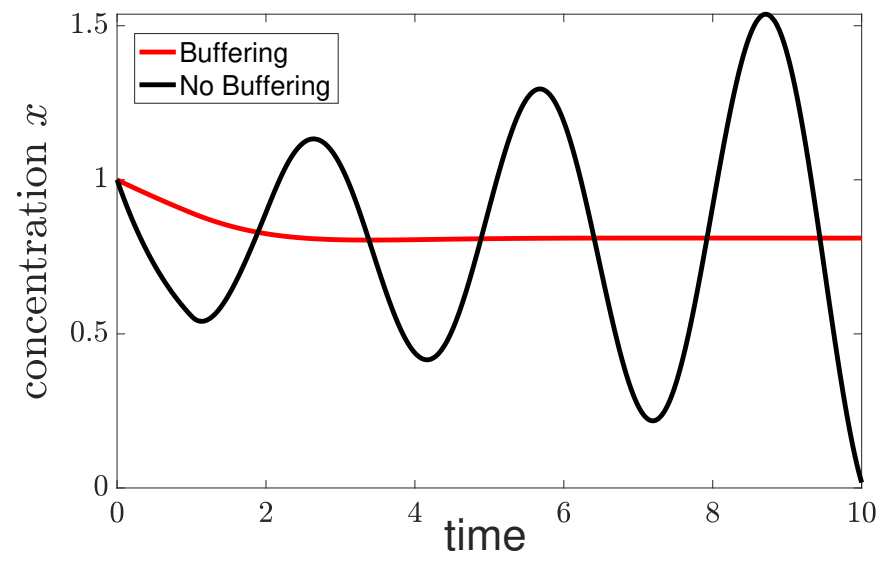

FIG. S1. The parameters are $B_{x}=0$ and $5, \tau=1$ (delay), $\gamma_{p}=1, \gamma_{w}=0$ and $h=2.7$. 


\section{SI2. STABILITY OF ANTITHETIC INTEGRAL FEEDBACK WITH BUFFERING}

\section{SI2.1. Stability of Antithetic Integral Feedback with Rapid Buffering: All Species}

In this section, we analyse the stabilising effect of buffering (without degradation) on antithetic integral feedback. We base our studies on a simple model involving the antithetic integral feedback (without buffering) ${ }^{5}$. Consider

$$
\begin{aligned}
& \dot{x}_{1}=\theta_{1} z_{1}-\gamma_{p} x_{1} \\
& \dot{x}_{2}=k x_{1}-\gamma_{p} x_{2} \\
& \dot{z}_{1}=\mu-\eta z_{1} z_{2} \\
& \dot{z}_{2}=\theta_{2} x_{2}-\eta z_{1} z_{2}
\end{aligned}
$$

where $x_{2}$ is the output concentration being controlled, $x_{1}$ is another concentration in the process being controlled, and $z_{1}$ and $z_{2}$ represent the molecular species involved in the perfect adaptation mechanism.

We next introduce buffering to (S4). We show how the model reduction method described in SI1 can be used to simplify the model for one case. We then use the same method for all buffers.

As a first case, we introduce buffering at the controlled variable $x_{2}$ and simplify the model by assuming that the buffering reactions rapidly reach equilibrium. With buffering at $x_{2}$, we have

$$
\begin{aligned}
\dot{x}_{1} & =\theta_{1} z_{1}-\gamma_{p} x_{1} \\
\dot{x}_{2} & =k x_{1}-\gamma_{p} x-\gamma_{p} x_{2}-b_{x} x+b_{w} w \\
\dot{z}_{1} & =\mu-\eta z_{1} z_{2} \\
\dot{z}_{2} & =\theta_{2} x_{2}-\eta z_{1} z_{2} \\
\dot{w}_{x} & =b_{x} x_{2}-b_{w} w_{x}
\end{aligned}
$$

where $w_{x}$ is the buffering species at $x_{2}$ and $b_{x}, b_{w}$ are the kinetic rate constants for the buffering reactions. Although the buffering equilibrium ratio is defined in terms of the linearised model and deviations from steady state, we can use the same notation here for the nonlinear model as the buffering reactions are linear. If the buffering reaction is at equilibrium then

$$
b_{x} x_{2}=b_{w} w_{x}
$$

If we assume that the buffer rapidly reaches equilibrium then $w$ is at quasi-steady state and so

$$
w_{x}=B_{x} x_{2} \quad B_{x}=\frac{b_{x}}{b_{w}} .
$$

We note that although the buffer equilibrium ratio is defined in (S2) in terms deviations from steady state, we can use the buffer equilibrium ratios in the nonlinear model as the reaction rates are linear.

We set $x_{T}=w_{x}+x$ as the slow variable and so $x_{T}=\left(1+B_{x}\right) x_{2}$. Thus $\dot{x}_{T}=\left(1+B_{x}\right) \dot{x}_{2}$ and so

$$
\left(1+B_{x}\right) \dot{x}_{2}=k x_{1}-\gamma_{p} x_{2} \text {. }
$$

If we include buffering on $z_{1}, z_{2}, x_{2}$ and apply a similar model reduction by assuming rapid buffering, then we have

$$
\begin{aligned}
\dot{x}_{1} & =\theta_{1} z_{1}-\gamma_{p} x_{1} \\
\left(1+B_{x}\right) \dot{x}_{2} & =k x_{1}-\gamma_{p} x_{2} \\
\left(1+B_{1}\right) \dot{z}_{1} & =\mu-\eta z_{1} z_{2} \\
\left(1+B_{2}\right) \dot{z}_{2} & =\theta_{2} x_{2}-\eta z_{1} z_{2}
\end{aligned}
$$

where $B_{1}, B_{2}$ are the buffer equilibrium ratios of the buffers at $z_{1}$ and $z_{2}$ respectively.

\section{SI2.1.a. Steady State}

We first determine the steady states of the system, which is useful both for determining perfect adaptation and as a prerequisite for stability analysis. Using

$$
\left(1+B_{1}\right) \dot{z}_{1}-\left(1+B_{2}\right) \dot{z}_{2}=\mu-\theta_{2} x_{2}=0
$$


we can see that the steady state for the output is

$$
\bar{x}_{2}=\frac{\mu}{\theta_{2}} \text {. }
$$

The correspondence of this steady state with perfect adaptation is discussed further in Section $2 \& 3$ of the paper. The species $x_{1}, z_{1}$ and $z_{2}$ have the corresponding steady states

$$
\bar{x}_{1}=\frac{\mu \gamma_{p}}{\theta_{2} k}, \quad \bar{z}_{1}=\frac{\gamma_{p}^{2} \mu}{\theta_{1} \theta_{2} k}, \quad \bar{z}_{2}=\frac{\theta_{1} \theta_{2} k}{\eta \gamma_{p}^{2}} .
$$

\section{SI2.1.b. Linearised Model}

We next determine the effect of buffering on the stability of the model. We follow the methodology used by Olsman and colleagues ${ }^{5}$ to study the stability of antithetic integral feedback with the addition of buffering. Linearising the system (S6) about the steady states, we have

$$
\begin{aligned}
\Delta \dot{x}_{1} & =\theta_{1} \Delta z_{1}-\gamma_{p} \Delta x_{1} \\
\left(1+B_{x}\right) \Delta \dot{x}_{2} & =k \Delta x_{1}-\gamma_{p} \Delta x_{2} \\
\left(1+B_{1}\right) \Delta \dot{z}_{1} & =-\alpha \Delta z_{1}-\frac{\beta}{\alpha} \Delta z_{2} \\
\left(1+B_{2}\right) \Delta \dot{z}_{2} & =\theta_{2} \Delta x_{2}-\alpha \Delta z_{1}-\frac{\beta}{\alpha} \Delta z_{2}
\end{aligned}
$$

where $\Delta z_{1}=z_{1}-\bar{z}_{1}, \Delta z_{2}=z_{2}-\bar{z}_{2}, \Delta x_{1}=x_{1}-\bar{x}_{1}, \Delta x_{2}=x_{2}-\bar{x}_{2}$ are the deviations from steady state and

$$
\alpha=\frac{\theta_{1} \theta_{2} k}{\gamma_{p}^{2}}, \quad \beta=\eta \mu
$$

Taking the Laplace transform of (S7), we have

$$
\begin{aligned}
\left(s+\gamma_{p}\right) X_{1} & =\theta_{1} Z_{1} \\
\left(\left(1+B_{x}\right) s+\gamma_{p}\right) X_{2} & =k X_{1} \\
\left(\left(1+B_{1}\right) s+\alpha\right) Z_{1} & =-\frac{\beta}{\alpha} Z_{2} \\
\left(\left(1+B_{2}\right) s+\frac{\beta}{\alpha}\right) Z_{2} & =\theta_{2} X_{2}-\alpha Z_{1}
\end{aligned}
$$

where $Z_{1}=\mathcal{L}\left\{\Delta z_{1}\right\}, Z_{2}=\mathcal{L}\left\{\Delta z_{2}\right\}, X_{1}=\mathcal{L}\left\{\Delta x_{1}\right\}, X_{2}=\mathcal{L}\left\{\Delta x_{2}\right\}$ are the Laplace transforms of the time-domain concentration deviations. Substituting, we have

$$
\begin{aligned}
\left(s+\gamma_{p}\right)\left(\left(1+B_{x}\right) s+\gamma_{p}\right) X_{2} & =\theta_{1} k Z_{1} \\
\left(\left(1+B_{2}\right) s+\frac{\beta}{\alpha}\right)\left(\left(1+B_{1}\right) s+\alpha\right) Z_{1} & =-\frac{\beta}{\alpha}\left(\theta_{2} X_{2}-\alpha Z_{1}\right) .
\end{aligned}
$$

Simplifying, we have

$$
\left(1+B_{1}\right)\left(1+B_{2}\right)\left(s^{2}+\left(\frac{\beta}{\alpha\left(1+B_{2}\right)}+\frac{\alpha}{1+B_{1}}\right) s\right) Z_{1}=-\frac{\beta}{\alpha} \theta_{2} X_{2} .
$$

Substituting from (S9), we have

$$
\left[\left(1+B_{1}\right)\left(1+B_{2}\right) s\left(\left(1+B_{x}\right) s+\gamma_{p}\right)\left(s+\gamma_{p}\right)\left(s+\frac{\beta}{\alpha\left(1+B_{2}\right)}+\frac{\alpha}{1+B_{1}}\right)+\frac{\beta}{\alpha} \theta_{1} \theta_{2} k\right] X_{2}=0 .
$$

The characteristic equation used to analyse stability is thus

$$
s\left(\left(1+B_{x}\right) s+\gamma_{p}\right)\left(s+\gamma_{p}\right)\left(s+\frac{\beta}{\alpha\left(1+B_{2}\right)}+\frac{\alpha}{1+B_{1}}\right)+\frac{\beta \gamma_{p}^{2}}{\left(1+B_{1}\right)\left(1+B_{2}\right)}=0 .
$$




\section{SI2.1.c. Characterisation of roots}

Following the methodology used by Olsman and colleagues ${ }^{5}$, we first characterise the roots of (S10). If we substitute $s=\gamma_{p} \sigma$ then

$$
\sigma(1+\sigma)\left(1+\left(1+B_{x}\right) \sigma\right)\left(\sigma+\frac{\beta}{\gamma_{p} \alpha\left(1+B_{2}\right)}+\frac{\alpha}{\gamma_{p}\left(1+B_{1}\right)}\right)=-\frac{\beta}{\gamma_{p}^{2}\left(1+B_{1}\right)\left(1+B_{2}\right)} .
$$

Taking the limit of strong binding for the sequestration process in antithetic integral feedback $\left(\beta \gg \max \left\{\alpha^{2} \frac{\left(1+B_{2}\right)}{\left(1+B_{1}\right)}, \alpha \gamma_{p}\left(1+B_{2}\right)\right\}\right)$ then

$$
\sigma(1+\sigma)\left(1+\left(1+B_{x}\right) \sigma\right)\left(1+\sigma \frac{\gamma_{p} \alpha\left(1+B_{2}\right)}{\beta}\right)=-\frac{\alpha}{\gamma_{p}\left(1+B_{1}\right)} .
$$

It can be observed that there is a large, real root at $\sigma \approx-\frac{\beta}{\gamma_{p} \alpha\left(1+B_{2}\right)}$. We next examine the region $|\sigma| \ll \frac{\beta}{\gamma_{p} \alpha\left(1+B_{2}\right)}$, where we have

$$
\sigma(1+\sigma)\left(1+\left(1+B_{x}\right) \sigma\right)=-\frac{\alpha}{\gamma_{p}\left(1+B_{1}\right)}
$$

The magnitude and phase constraints are

$$
\begin{aligned}
& |\sigma||1+\sigma|\left|1+\left(1+B_{x}\right) \sigma\right|=\frac{\alpha}{\gamma_{p}\left(1+B_{1}\right)} \\
& \arg \sigma+\arg (1+\sigma)+\arg \left(1+\left(1+B_{x}\right) \sigma\right)=(1+2 k) \pi .
\end{aligned}
$$

If we assume that $\sigma$ is real and positive, then the LHS of the phase constraint is

$$
\arg \sigma+\arg (1+\sigma)+\arg \left(1+\left(1+B_{x}\right) \sigma\right)=0
$$

which contradicts. Thus unstable roots are not purely real.

If we assume that $\sigma$ is real and $-1 /\left(1+B_{x}\right)<\sigma<0$ then the LHS of the phase constraint is

$$
\arg \sigma+\arg (1+\sigma)+\arg \left(1+\left(1+B_{x}\right) \sigma\right)=\pi
$$

and so it is possible to have stable real roots. If we set $f=|\sigma||1+\sigma|\left|1+\left(1+B_{x}\right) \sigma\right|$ from the magnitude constraint, then there is a local maxim of $f$ at

$$
\sigma_{\max }=\frac{-\left(2+B_{x}\right)+\sqrt{1+B_{x}+B_{x}^{2}}}{3\left(1+B_{x}\right)} .
$$

Thus there are two stable real roots between $-1 /\left(1+B_{x}\right)<\sigma<0$ if

$$
f\left(\sigma_{\max }\right)>\frac{\alpha}{\gamma_{p}\left(1+B_{1}\right)}
$$

as $f\left(\sigma_{\max }\right)$ is larger than the RHS of the magnitude constraint. There is a bifurcation at the boundary $f\left(\sigma_{\max }\right)=\frac{\alpha}{\gamma_{p}\left(1+B_{1}\right)}$ resulting in a pair of complex conjugate roots if

$$
f\left(\sigma_{\max }\right)<\frac{\alpha}{\gamma_{p}\left(1+B_{1}\right)} .
$$

For $-1<\sigma<-1 /\left(1+B_{x}\right)$ then

$$
\arg \sigma+\arg (1+\sigma)+\arg \left(1+\left(1+B_{x}\right) \sigma\right)=2 \pi
$$

which violates the phase constraints. For $\sigma<-1$ then

$$
\arg \sigma+\arg (1+\sigma)+\arg \left(1+\left(1+B_{x}\right) \sigma\right)=3 \pi
$$

which meets the phase constraints. Thus for the stability boundary with strong binding there is a negative real root and a complex pair of roots in the region $|\sigma| \ll \frac{\beta}{\gamma_{p} \alpha\left(1+B_{2}\right)}$, as well as one large negative root. 


\section{SI2.1.d. Stability Conditions}

We next determine the stability boundary, where roots of the characteristic equation have zero real parts. Substituting $s=i \omega \gamma_{p}$, we have

$$
\gamma_{p}^{4} i \omega(1+i \omega)\left(1+\left(1+B_{x}\right) i \omega\right)\left(i \omega+\left(\frac{\beta}{\alpha \gamma_{p}\left(1+B_{2}\right)}+\frac{\alpha}{\gamma_{p}\left(1+B_{1}\right)}\right)\right)=-\frac{\beta \gamma_{p}^{2}}{\left(1+B_{1}\right)\left(1+B_{2}\right)} .
$$

From these equations, the magnitude and phase constraint are

$$
\begin{aligned}
& \omega\left(1+\omega^{2}\right)^{0.5}\left(1+\left(1+B_{x}\right)^{2} \omega^{2}\right)^{0.5}\left(\omega^{2}+\phi^{2}\right)^{0.5}=\frac{\beta}{\gamma_{p}^{2}\left(1+B_{1}\right)\left(1+B_{2}\right)} \\
& \tan ^{-1}(\omega)+\tan ^{-1}\left(\left(1+B_{x}\right) \omega\right)+\tan ^{-1}\left(\frac{\omega}{\phi}\right)=\frac{\pi}{2}+2 k \pi \\
& \phi=\frac{\beta}{\alpha \gamma_{p}\left(1+B_{2}\right)}+\frac{\alpha}{\gamma_{p}\left(1+B_{1}\right)}
\end{aligned}
$$

for some integer $k$. Using the strong binding assumption $\left(\beta \gg \max \left\{\alpha^{2} \frac{\left(1+B_{2}\right)}{\left(1+B_{1}\right)}, \alpha \gamma_{p}\left(1+B_{2}\right)\right\}\right)$ then from above $|\omega| \ll \frac{\beta\left(1+B_{1}\right)}{\gamma_{p} \alpha\left(1+B_{2}\right)}$ and so

$$
\begin{aligned}
& \omega\left(1+\omega^{2}\right)^{0.5}\left(1+\left(1+B_{x}\right)^{2} \omega^{2}\right)^{0.5}=\frac{\alpha}{\gamma_{p}\left(1+B_{1}\right)} \\
& \tan ^{-1}(\omega)+\tan ^{-1}\left(\left(1+B_{x}\right) \omega\right)=\frac{\pi}{2}+2 k \pi
\end{aligned}
$$

Rewriting the phase constraint, we have

$$
\tan ^{-1}(\omega)-\frac{\pi}{4}=\frac{\pi}{4}-\tan ^{-1}\left(\left(1+B_{x}\right) \omega\right) .
$$

Applying $\tan (\cdot)$ and trigonometric identities to both sides, we have

$$
\frac{\omega-1}{\omega+1}=\frac{1-\left(1+B_{x}\right) \omega}{1+\left(1+B_{x}\right) \omega} .
$$

Solving, we have

$$
\omega=\frac{1}{\sqrt{1+B_{x}}}
$$

Thus the stability boundary occurs at

$$
\gamma_{p}=\frac{\alpha}{\left(1+B_{1}\right)} \frac{1+B_{x}}{2+B_{x}}
$$

From above, we know that all roots are real and stable if $\alpha /\left(\gamma_{p}\left(1+B_{1}\right)\right)$ is sufficiently small, and so the stability condition is

$$
\gamma_{p}>\frac{\alpha}{\left(1+B_{1}\right)} \frac{1+B_{x}}{2+B_{x}}
$$

or

$$
\gamma_{p}^{3}>\frac{\theta_{1} \theta_{2} k}{\left(1+B_{1}\right)} \frac{1+B_{x}}{2+B_{x}}
$$

Thus increasing $B_{1}$ improves stability and increasing $B_{2}$ has no effect on stability. Further, increasing $B_{x}$ worsens stability, although this effect saturates as $B_{x}$ increases. 


\section{S12.2. Stability of Antithetic Feedback with Non-Rapid Buffering at $z_{1}$}

In this section, we analyse the ability of non-rapid buffering at $z_{1}$ to stabilise antithetic integral feedback. We use the model

$$
\begin{aligned}
\dot{x}_{1} & =\theta_{1} z_{1}-\gamma_{p} x_{1} \\
\dot{x}_{2} & =k x_{1}-\gamma_{p} x_{2} \\
\dot{z}_{1} & =\mu-\eta z_{1} z_{2}-b_{z} z_{1}+b_{w} w \\
\dot{z}_{2} & =\theta_{2} x_{2}-\eta z_{1} z_{2} \\
\dot{w} & =b_{z} z_{1}-b_{w} w .
\end{aligned}
$$

where the buffer $w$ is not assumed to rapidly reach equilbrium. As a result, the model cannot be reduced in a similar manner to SI2.1. The steady state for (S13) is identical to the rapid case in SI2.1. The linearisation is

$$
\begin{aligned}
\Delta \dot{x}_{1} & =\theta_{1} \Delta z_{1}-\gamma_{p} \Delta x_{1} \\
\Delta \dot{x}_{2} & =k \Delta x_{1}-\gamma_{p} \Delta x_{2} \\
\Delta \dot{z}_{1} & =-\eta \bar{z}_{2} \Delta z_{1}-\eta \bar{z}_{1} \Delta z_{2}-b_{z} \Delta z_{1}+b_{w} \Delta w \\
\Delta \dot{z}_{2} & =\theta_{2} \Delta x_{2}-\eta \bar{z}_{2} \Delta z_{1}-\eta \bar{z}_{1} \Delta z_{2} \\
\Delta \dot{w} & =b_{z} \Delta z_{1}-b_{w} \Delta w .
\end{aligned}
$$

This system can be rewritten

$$
\begin{aligned}
\Delta \dot{x}_{1} & =\theta_{1} \Delta z_{1}-\gamma_{p} \Delta x_{1} \\
\Delta \dot{x}_{2} & =k \Delta x_{1}-\gamma_{p} \Delta x_{2} \\
\Delta \dot{z}_{1} & =-\alpha \Delta z_{1}-\frac{\beta}{\alpha} \Delta z_{2}-b_{z} \Delta z_{1}+b_{w} \Delta w \\
\Delta \dot{z}_{2} & =\theta_{2} \Delta x_{2}-\alpha \Delta z_{1}-\frac{\beta}{\alpha} \Delta z_{2} \\
\Delta \dot{w} & =b_{z} \Delta z_{1}-b_{w} \Delta w
\end{aligned}
$$

where

$$
\alpha=\frac{\theta_{1} \theta_{2} k}{\gamma_{p}^{2}}, \quad \beta=\eta \mu .
$$

Taking the Laplace transform of $\Delta \dot{w}=b_{z} \Delta z_{1}-b_{w} \Delta w$, we have

$$
W=\frac{b_{z}}{s+b_{w}} Z_{1}
$$

where $W$ and $Z_{1}$ are the Laplace transforms of $w$ and $z_{1}$. We have

$$
\begin{aligned}
-b_{z} Z_{1}+b_{w} W & =-b_{z} Z_{1}+b_{w} \frac{b_{z}}{s+b_{w}} Z_{1} \\
& =-B_{1} \frac{s}{1+\frac{s}{b_{w}}} Z_{1} \\
& =-C_{b}(s) Z_{1}
\end{aligned}
$$

where

$$
C_{b}=B_{1} \frac{s}{1+\frac{s}{b_{w}}} .
$$

Taking the Laplace transform of (S14), we have

$$
\begin{aligned}
& \left(s+\gamma_{p}\right) X_{1}=\theta_{1} Z_{1} \\
& \left(s+\gamma_{p}\right) X_{2}=k X_{1} \\
& \left(s+\alpha+C_{b}\right) Z_{1}=-\frac{\beta}{\alpha} Z_{2} \\
& \left(s+\frac{\beta}{\alpha}\right) Z_{2}=\theta_{2} X_{2}-\alpha Z_{1} .
\end{aligned}
$$


Combining, we have

$$
\begin{aligned}
& \left(s+\gamma_{p}\right)^{2} X_{2}=\theta_{1} k Z_{1} \\
& \left(s+\alpha+C_{b}\right)\left(s+\frac{\beta}{\alpha}\right) Z_{1}=-\frac{\beta}{\alpha}\left(\theta_{2} X_{2}-\alpha Z_{1}\right)
\end{aligned}
$$

Simplifying, we have

$$
\left[s\left(s+\alpha+\frac{\beta}{\alpha}\right)+C_{b}\left(s+\frac{\beta}{\alpha}\right)\right] Z_{1}=-\frac{\beta}{\alpha} \theta_{2} X_{2}
$$

Taking the strong antithetic binding limit of the sequestration mechanism $\left(\beta \gg \max \left\{\alpha^{2}, \gamma_{p} \alpha\right\}\right)$, we have

$$
\left(s+C_{b}\right)\left(s+\frac{\beta}{\alpha}\right) Z_{1}=-\frac{\beta}{\alpha} \theta_{2} X_{2}
$$

and so

$$
\left(s+\gamma_{p}\right)^{2}\left[\left(s+C_{b}\right)\left(s+\frac{\beta}{\alpha}\right)\right] X_{2}=-\beta \gamma_{p}^{2} X_{2}
$$

Rewriting $C_{b}$, we have

$$
\left[\left(s+\gamma_{p}\right)^{2} s \frac{1+B_{1}+\frac{s}{b_{w}}}{1+\frac{s}{b_{w}}}\left(s+\frac{\beta}{\alpha}\right)+\beta \gamma_{p}^{2}\right] X_{2}=0
$$

and so the characteristic equation is

$$
\left(s+\gamma_{p}\right)^{2} s\left(1+B_{1}+\frac{s}{b_{w}}\right)\left(s+\frac{\beta}{\alpha}\right)+\beta \gamma_{p}^{2}\left(1+\frac{s}{b_{w}}\right)=0 .
$$

Substituting $s=i \omega \gamma_{p}$, we have

$$
\left(1+B_{1}+i \omega \frac{\gamma_{p}}{b_{w}}\right)(1+i \omega)^{2} i \omega\left(i \omega+\frac{\beta}{\gamma_{p} \alpha}\right)+\frac{\beta}{\gamma_{p}^{2}}\left(1+i \omega \frac{\gamma_{p}}{b_{w}}\right)=0 .
$$

Taking the strong antithetic binding limit where $|i \omega| \ll \frac{\beta}{\gamma_{p} \alpha}$, we have

$$
\left(1+B_{1}+i \omega \frac{\gamma_{p}}{b_{w}}\right)(1+i \omega)^{2} i \omega=-\frac{\alpha}{\gamma_{p}}\left(1+i \omega \frac{\gamma_{p}}{b_{w}}\right) .
$$

The magnitude constraint is

$$
\left(1+B_{1}\right)\left(1+\frac{\gamma_{p}^{2}}{b_{w}^{2}} \frac{\omega^{2}}{\left(1+B_{1}\right)^{2}}\right)^{0.5}\left(1+\omega^{2}\right) \omega=\frac{\alpha}{\gamma_{p}}\left(1+\frac{\gamma_{p}^{2}}{b_{w}^{2}} \omega^{2}\right)^{0.5}
$$

and the phase constraint is

$$
\tan ^{-1}\left(\frac{\gamma_{p}}{b_{w}} \frac{\omega}{1+B_{1}}\right)+2 \tan ^{-1}(\omega)+\frac{\pi}{2}=\tan ^{-1}\left(\frac{\gamma_{p}}{b_{w}} \omega\right)+\pi+2 k \pi .
$$

for some integer $k$. Using trigonometric identities, we have

$$
\tan ^{-1}\left(\frac{2 \omega}{1-\omega^{2}}\right)-\frac{\pi}{4}=\tan ^{-1}\left(\frac{\frac{B_{1}}{1+B_{1}} \frac{\gamma_{p}}{b_{w}} \omega}{1+\frac{1}{1+B_{1}} \frac{\gamma_{p}^{2}}{b_{w}^{2}} \omega^{2}}\right)+\frac{\pi}{4}+2 k \pi
$$


This can be simplified to

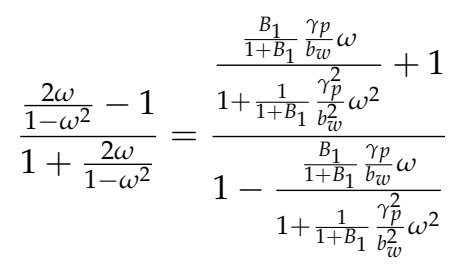

or

$$
\frac{2 \omega-1+\omega^{2}}{1-\omega^{2}+2 \omega}=\frac{1+\frac{1}{1+B_{1}} \frac{\gamma_{p}^{2}}{b_{w}^{2}} \omega^{2}+\frac{B_{1}}{1+B_{1}} \frac{\gamma_{p}}{b_{w}} \omega}{1+\frac{1}{1+B_{1}} \frac{\gamma_{p}^{2}}{b_{w}^{2}} \omega^{2}-\frac{B_{1}}{1+B_{1}} \frac{\gamma_{p}}{b_{w}} \omega}
$$

We have

$$
\begin{aligned}
& \left(2 \omega-1+\omega^{2}\right)\left(1+\frac{1}{1+B_{1}} \frac{\gamma_{p}^{2}}{b_{w}^{2}} \omega^{2}-\frac{B_{1}}{1+B_{1}} \frac{\gamma_{p}}{b_{w}} \omega\right) \\
& =\left(1+\frac{1}{1+B_{1}} \frac{\gamma_{p}^{2}}{b_{w}^{2}} \omega^{2}+\frac{B_{1}}{1+B_{1}} \frac{\gamma_{p}}{b_{w}} \omega\right)\left(1-\omega^{2}+2 \omega\right) .
\end{aligned}
$$

Setting

$$
\begin{aligned}
& a_{1}=\frac{B_{1}}{1+B_{1}} \frac{\gamma_{p}}{b_{w}} \\
& a_{2}=\frac{1}{1+B_{1}} \frac{\gamma_{p}^{2}}{b_{w}^{2}}
\end{aligned}
$$

we have

$$
\left(2 \omega-1+\omega^{2}\right)\left(1+a_{2} \omega^{2}-a_{1} \omega\right)=\left(1+a_{2} \omega^{2}+a_{1} \omega\right)\left(1-\omega^{2}+2 \omega\right)
$$

Matching coefficients, we have

$$
2 a_{1} \omega^{2}+\left(1-\omega^{2}\right)\left(1+a_{2} \omega^{2}\right)=0
$$

and thus

$$
a_{2} \omega^{4}+\left(1-2 a_{1}-a_{2}\right) \omega^{2}-1=0
$$

Solving for $\omega^{2}$, we have the stability constraint

$$
\gamma_{p}>\frac{\alpha}{\left(1+B_{1}\right)} \frac{1}{\left(1+\omega^{2}\right) \omega}\left(\frac{1+\frac{\gamma_{p}^{2}}{b_{w}^{2}} \omega^{2}}{1+\frac{\gamma_{p}^{2}}{b_{w}^{2}} \frac{\omega^{2}}{\left(1+B_{1}\right)^{2}}}\right)^{0.5}
$$

where

$$
\omega^{2}=\sqrt{\left(\frac{1}{2 a_{2}}-\frac{a_{1}}{a_{2}}-\frac{1}{2}\right)^{2}+\frac{1}{a_{2}}}-\left(\frac{1}{2 a_{2}}-\frac{a_{1}}{a_{2}}-\frac{1}{2}\right)
$$

which can be rewritten as

$$
\gamma_{p}^{3}>\frac{\theta_{1} \theta_{2} k}{\left(1+B_{1}\right)} \underbrace{\frac{1}{\left(1+\omega^{2}\right) \omega}\left(\frac{1+\frac{\gamma_{p}^{2}}{b_{w}^{2}} \omega^{2}}{1+\frac{\gamma_{p}^{2}}{b_{w}^{2}} \frac{\omega^{2}}{\left(1+B_{1}\right)^{2}}}\right)^{0.5}}_{\mathrm{F}}
$$




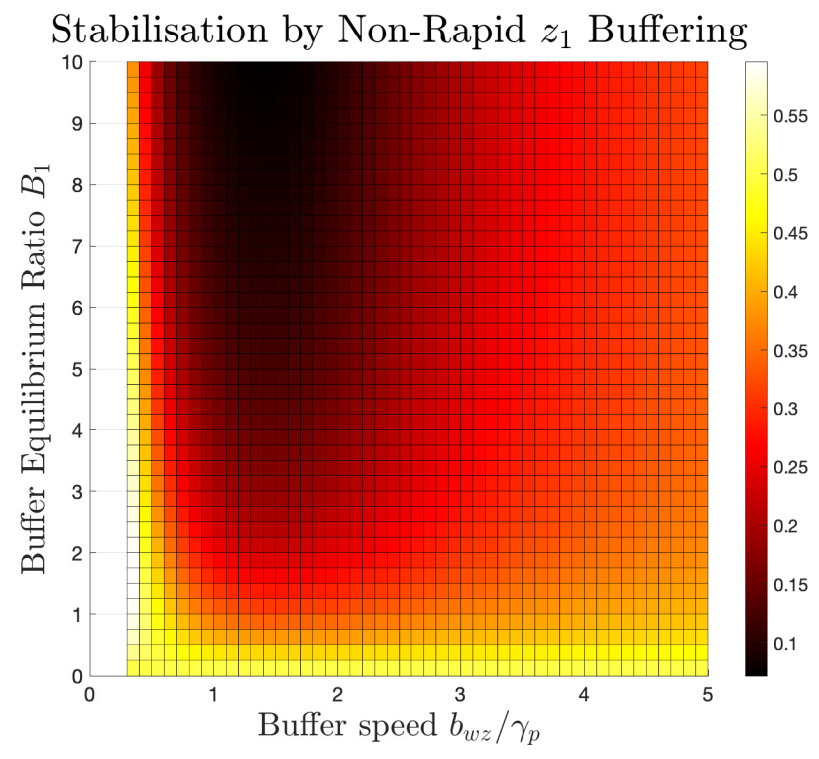

FIG. S2. Stabilisation effect F in (S18) where smaller implies improved stability.

$$
\omega^{2}=\sqrt{\left(\left(1+B_{1}\right) \frac{b_{w}^{2}}{2 \gamma_{p}^{2}}-B_{1} \frac{b_{w}}{\gamma_{p}}-\frac{1}{2}\right)^{2}+\left(1+B_{1}\right) \frac{b_{w}^{2}}{\gamma_{p}^{2}}}-\left(\left(1+B_{1}\right) \frac{b_{w}^{2}}{2 \gamma_{p}^{2}}-B_{1} \frac{b_{w}}{\gamma_{p}}-\frac{1}{2}\right)
$$

where $F$, which is the stabilisation effect independent of the integral gain, can be seen as a function of $B_{1}$ and $\frac{b_{w}}{\gamma_{p}}$ in Figure S2.

For the special case that $b_{w}=\gamma_{p}$ then we have

$$
\omega^{2}=1+B_{1}
$$

and so

$$
\gamma_{p}^{3}>\frac{\theta_{1} \theta_{2} k}{\left(1+B_{1}\right)} \frac{1}{\left(2+B_{1}\right)}
$$

\section{S12.3. Stability of Antithetic Feedback with Non-Rapid Buffering at $x_{2}$}

In this section, we analyse the ability of non-rapid buffering at $x_{2}$ to stabilise antithetic integral feedback. We use the model

$$
\begin{aligned}
\dot{x}_{1} & =\theta_{1} z_{1}-\gamma_{p} x_{1} \\
\dot{x}_{2} & =k x_{1}-\gamma_{p} x_{2}-b_{x} x_{2}+b_{w} w \\
\dot{w} & =b_{x} x_{2}-b_{w} w \\
\dot{z}_{1} & =\mu-\eta z_{1} z_{2} \\
\dot{z}_{2} & =\theta_{2} x_{2}-\eta z_{1} z_{2} .
\end{aligned}
$$

where the buffer $w$ is not assumed to rapidly reach equilbrium. As a result, the model cannot be reduced in a similar manner to SI2.1. The steady state for (S19) is identical to the rapid case in SI2.1. The linearisation is

$$
\begin{aligned}
\Delta \dot{x}_{1} & =\theta_{1} \Delta z_{1}-\gamma_{p} \Delta x_{1} \\
\Delta \dot{x}_{2} & =k \Delta x_{1}-\gamma_{p} \Delta x_{2}-b_{x} \Delta x_{2}+b_{w} \Delta w \\
\Delta \dot{w} & =b_{x} \Delta x_{2}-b_{w} \Delta w \\
\Delta \dot{z}_{1} & =-\eta \bar{z}_{2} \Delta z_{1}-\eta \bar{z}_{1} \Delta z_{2} \\
\Delta \dot{z}_{2} & =\theta_{2} \Delta x_{2}-\eta \bar{z}_{2} \Delta z_{1}-\eta \bar{z}_{1} \Delta z_{2} .
\end{aligned}
$$


This system can be rewritten

$$
\begin{aligned}
\Delta \dot{x}_{1} & =\theta_{1} \Delta z_{1}-\gamma_{p} \Delta x_{1} \\
\Delta \dot{x}_{2} & =k \Delta x_{1}-\gamma_{p} \Delta x_{2}-b_{x} \Delta x_{2}+b_{w} \Delta w \\
\Delta \dot{w} & =b_{x} \Delta x_{2}-b_{w} \Delta w \\
\Delta \dot{z}_{1} & =-\alpha \Delta z_{1}-\frac{\beta}{\alpha} \Delta z_{2} \\
\Delta \dot{z}_{2} & =\theta_{2} \Delta x_{2}-\alpha \Delta z_{1}-\frac{\beta}{\alpha} \Delta z_{2}
\end{aligned}
$$

where

$$
\alpha=\frac{\theta_{1} \theta_{2} k}{\gamma_{p}^{2}}, \quad \beta=\eta \mu
$$

Taking the Laplace transform of $\Delta \dot{w}=b_{z} \Delta z_{1}-b_{w} \Delta w$, we have

$$
W=\frac{b_{z}}{s+b_{w}} Z_{1}
$$

where $W$ and $Z_{1}$ are the Laplace transforms of $w$ and $z_{1}$. We have

$$
-b_{x} X_{2}+b_{w} W=-B_{x} \frac{s}{1+\frac{s}{b_{w}}} X_{2}=-C_{b}(s) X_{2}
$$

where

$$
C_{b}=B_{x} \frac{s}{1+\frac{s}{b_{w}}}
$$

Taking the Laplace transform of (S20), we have

$$
\begin{aligned}
& \left(s+\gamma_{p}\right) X_{1}=\theta_{1} Z_{1} \\
& \left(s+\gamma_{p}+C_{b}\right) X_{2}=k X_{1} \\
& (s+\alpha) Z_{1}=-\frac{\beta}{\alpha} Z_{2} \\
& \left(s+\frac{\beta}{\alpha}\right) Z_{2}=\theta_{2} X_{2}-\alpha Z_{1} .
\end{aligned}
$$

Combining, we have

$$
\begin{aligned}
& \left(s+\gamma_{p}\right)\left(s+\gamma_{p}+C_{b}\right) X_{2}=\theta_{1} k Z_{1} \\
& (s+\alpha)\left(s+\frac{\beta}{\alpha}\right) Z_{1}=-\frac{\beta}{\alpha}\left(\theta_{2} X_{2}-\alpha Z_{1}\right)
\end{aligned}
$$

Simplifying, we have

$$
s\left(s+\alpha+\frac{\beta}{\alpha}\right) Z_{1}=-\frac{\beta}{\alpha} \theta_{2} X_{2} .
$$

Taking the strong antithetic binding limit of the sequestration mechanism $\left(\beta \gg \max \left\{\alpha^{2}, \gamma_{p} \alpha\right\}\right)$, we have

$$
s\left(s+\frac{\beta}{\alpha}\right) Z_{1}=-\frac{\beta}{\alpha} \theta_{2} X_{2}
$$

and so

$$
\left(s+\gamma_{p}\right)\left(s+\gamma_{p}+C_{b}\right) s\left(s+\frac{\beta}{\alpha}\right) X_{2}=-\beta \gamma_{p}^{2} X_{2}
$$


Rewriting $C_{b}$, we have

$$
\left(s+\gamma_{p}\right)\left(s+\gamma_{p}+B_{x} \frac{s}{1+\frac{s}{b_{w}}}\right) s\left(s+\frac{\beta}{\alpha}\right) X_{2}=-\beta \gamma_{p}^{2} X_{2}
$$

and so the characteristic equation is

$$
\left(s+\gamma_{p}\right)\left(\left(s+\gamma_{p}\right)\left(1+\frac{s}{b_{w}}\right)+B_{x} s\right) s\left(s+\frac{\beta}{\alpha}\right)=-\beta \gamma_{p}^{2}\left(1+\frac{s}{b_{w}}\right) .
$$

Substituting $s=i \omega \gamma_{p}$, we have

$$
(1+i \omega)\left((1+i \omega)\left(1+i \omega \frac{\gamma_{p}}{b_{w}}\right)+i \omega B_{x}\right) i \omega\left(i \omega+\frac{\beta}{\gamma_{p} \alpha}\right)=-\frac{\beta}{\gamma_{p}^{2}}\left(1+i \omega \frac{\gamma_{p}}{b_{w}}\right)
$$

Taking the strong antithetic binding limit where $|i \omega| \ll \frac{\beta}{\gamma_{p} \alpha}$, we have

$$
\left(1-\frac{\gamma_{p}}{b_{w}} \omega^{2}+i \omega\left(1+B_{x}+\frac{\gamma_{p}}{b_{w}}\right)\right)(1+i \omega) i \omega=-\frac{\alpha}{\gamma_{p}}\left(1+i \omega \frac{\gamma_{p}}{b_{w}}\right) .
$$

The magnitude constraint is

$$
\left(\left(\left(1-\frac{\gamma_{p}}{b_{w}} \omega^{2}\right)^{2}+\omega^{2}\left(1+B_{x}+\frac{\gamma_{p}}{b_{w}}\right)^{2}\right)^{0.5}\left(1+\omega^{2}\right)^{0.5} \omega=\frac{\alpha}{\gamma_{p}}\left(1+\frac{\gamma_{p}^{2}}{b_{w}^{2}} \omega^{2}\right)^{0.5}\right.
$$

and the phase constraint is

$$
\tan ^{-1}\left(\frac{\omega\left(1+B_{x}+\frac{\gamma_{p}}{b_{w}}\right)}{1-\frac{\gamma_{p}}{b_{w}} \omega^{2}}\right)+\tan ^{-1}(\omega)+\frac{\pi}{2}=\tan ^{-1}\left(\frac{\gamma_{p}}{b_{w}} \omega\right)+\pi+2 k \pi .
$$

for some integer $k$. Using trigonometric identities and rearranging, we have

$$
\tan ^{-1}\left(\frac{\omega\left(1+B_{x}+\frac{\gamma_{p}}{b_{w}}\right)}{1-\frac{\gamma_{p}}{b_{w}} \omega^{2}}\right)-\frac{\pi}{4}=\tan ^{-1}\left(\frac{\left(\frac{\gamma_{p}}{b_{w}}-1\right) \omega}{1+\frac{\gamma_{p}}{b_{w}} \omega^{2}}\right)+\frac{\pi}{4}+2 k \pi .
$$

Taking the tangent of both sides, we have

$$
\frac{\frac{\omega\left(1+B_{x}+\frac{\gamma p}{b_{w}}\right)}{1-\frac{\gamma p}{b_{w}} \omega^{2}}-1}{1+\frac{\omega\left(1+B_{x}+\frac{\gamma p}{b_{w}}\right)}{1-\frac{\gamma p}{b_{w}} \omega^{2}}}=\frac{\frac{\left(\frac{\gamma p}{b_{w}}-1\right) \omega}{1+\frac{\gamma p}{b_{w}} \omega^{2}}+1}{1-\frac{\left(\frac{\gamma p}{b_{w}}-1\right) \omega}{1+\frac{\gamma p}{b_{w}} \omega^{2}}}
$$

Multiplying out fractions, we have

$$
\frac{\omega\left(1+B_{x}+\frac{\gamma_{p}}{b_{w}}\right)-\left(1-\frac{\gamma_{p}}{b_{w}} \omega^{2}\right)}{1-\frac{\gamma_{p}}{b_{w}} \omega^{2}+\omega\left(1+B_{x}+\frac{\gamma_{p}}{b_{w}}\right)}=\frac{\left(\frac{\gamma_{p}}{b_{w}}-1\right) \omega+1+\frac{\gamma_{p}}{b_{w}} \omega^{2}}{1+\frac{\gamma_{p}}{b_{w}} \omega^{2}-\left(\frac{\gamma_{p}}{b_{w}}-1\right) \omega} .
$$

Simplifying, we have

$$
\frac{\gamma_{p}^{2}}{b_{w}^{2}} \omega^{4}+\left(1+B_{x}+\frac{\gamma_{p}}{b_{w}}\right)\left(1-\frac{\gamma_{p}}{b_{w}}\right) \omega^{2}-1=0
$$




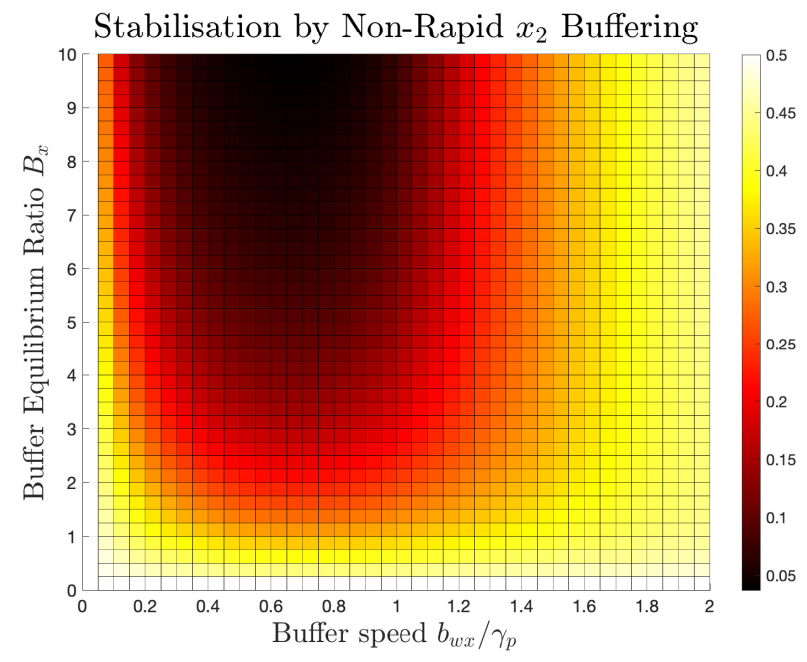

FIG. S3. Stabilisation effect F in (S22) where smaller implies improved stability.

Solving for $\omega^{2}$ and using the same approach as in previous sections, the magnitude constraint leads to the stability

$$
\begin{aligned}
& \gamma_{p}^{3}>\theta_{1} \theta_{2} k \underbrace{\frac{1}{\left(1+\omega^{2}\right)^{0.5} \omega}(\underbrace{1+\frac{\gamma_{p}^{2}}{b_{w}^{2}} \omega^{2}})^{0.5}}_{\mathrm{F}} \\
& \left.\omega^{2}=\frac{b_{w}^{2}}{2 \gamma_{p}^{2}}\left[\sqrt{\left(1-\frac{\gamma_{p}}{b_{w}} \omega^{2}\right)^{2}+\omega^{2}\left(1+B_{x}+\frac{\gamma_{p}}{b_{w}}\right)^{2}}\right)^{\left(1+B_{x}+\frac{\gamma_{p}}{b_{w}}\right)^{2}\left(1-\frac{\gamma_{p}}{b_{w}}\right)^{2}+4 \frac{\gamma_{p}^{2}}{b_{w}^{2}}}-\left(1+B_{x}+\frac{\gamma_{p}}{b_{w}}\right)\left(1-\frac{\gamma_{p}}{b_{w}}\right)\right] .
\end{aligned}
$$

where $F$, which is the stabilisation effect independent of the integral gain, can be seen as a function of $B_{x}$ and $\frac{b_{w}}{\gamma_{p}}$ in Figure S3.

For the special case where we set $b_{w}=\gamma_{p}$ then we have $\omega^{2}=1$ and so the stability constraint is

$$
\gamma_{p}^{3}>\frac{\theta_{1} \theta_{2} k}{2+B_{x}}
$$

\section{S12.4. Stability of Antithetic Feedback: Rapid $x_{2}$ Buffering with Degradation}

In this section, we analyse the stabilising effect of buffering at the output species $x_{2}$ on antithetic integral feedback, where the buffering can be degraded. Consider the model (S5) with buffering at $x_{2}$ that is degraded

$$
\begin{aligned}
\dot{x}_{1} & =\theta_{1} z_{1}-\gamma_{p} x_{1} \\
\dot{x}_{2} & =k x_{1}-\gamma_{p} x_{2}-b_{x} x_{2}+b_{w} w_{x} \\
\dot{w}_{x} & =b_{x} x_{2}-b_{w} w_{x}-\gamma_{x} w_{x} \\
\dot{z}_{1} & =\mu-\eta z_{1} z_{2} \\
\dot{z}_{2} & =\theta_{2} x_{2}-\eta z_{1} z_{2}
\end{aligned}
$$

where $w_{x}$ is the buffering species of $x_{2}$, and $b_{x}, b_{w}$ are the kinetic rates for the buffering reactions. If we assume rapid buffering such that $w_{x}+x_{2}$ is the slow variable, $w_{x}=B_{x} x_{2}$ and $B_{x}=\frac{b_{x}}{b_{w}+\gamma_{x}}$, and use the methodology from 
SI1 and SI2.1 then we have the reduced model

$$
\begin{aligned}
\dot{x}_{1} & =\theta_{1} z_{1}-\gamma_{p} x_{1} \\
\left(1+B_{x}\right) \dot{x}_{2} & =k x_{1}-\left(\gamma_{p}+B_{x} \gamma_{x}\right) x_{2} \\
\dot{z}_{1} & =\mu-\eta z_{1} z_{2} \\
\dot{z}_{2} & =\theta_{2} x_{2}-\eta z_{1} z_{2} .
\end{aligned}
$$

\section{S12.4.a. Steady State Analysis}

We next analyse the steady state. We have

$$
\dot{z}_{1}-\dot{z}_{2}=\mu-\theta_{2} x_{2}
$$

and so the steady state of the output is

$$
x_{2}=\frac{\mu}{\theta_{2}}
$$

We also have corresponding steady states

$$
\begin{aligned}
& x_{1}=\frac{\gamma_{p}}{k}\left(1+B_{x} \frac{\gamma_{x}}{\gamma_{p}}\right) \frac{\mu}{\theta_{2}} \\
& z_{1}=\frac{\gamma_{p}}{\theta_{1}} x_{1}=\frac{\gamma_{p}^{2} \mu}{\theta_{1} k \theta_{2}}\left(1+B_{x} \frac{\gamma_{x}}{\gamma_{p}}\right)=\frac{\mu}{\alpha}\left(1+B_{x} \frac{\gamma_{x}}{\gamma_{p}}\right) \\
& z_{2}=\frac{1}{\eta} \frac{\mu}{z_{1}}=\frac{\alpha}{\eta\left(1+B_{x} \frac{\gamma_{x}}{\gamma_{p}}\right)}
\end{aligned}
$$

where $\alpha=\frac{\theta_{1} \theta_{2} k}{\gamma_{p}^{2}}$.

\section{SI2.4.b. Linearised Model}

We next analyse the stability of the system. If we linearise about the steady states, we have

$$
\begin{aligned}
\Delta \dot{x}_{1} & =\theta_{1} \Delta z_{1}-\gamma_{p} \Delta x_{1} \\
\left(1+B_{x}\right) \Delta \dot{x}_{2} & =k \Delta x_{1}-\gamma_{p}\left(1+B_{x} \frac{\gamma_{x}}{\gamma_{p}}\right) \Delta x_{2} \\
\Delta \dot{z}_{1} & =-\eta \bar{z}_{2} \Delta z_{1}-\eta \bar{z}_{1} \Delta z_{2} \\
\Delta \dot{z}_{2} & =\theta_{2} \Delta x_{2}-\eta \bar{z}_{2} \Delta z_{1}-\eta \bar{z}_{1} \Delta z_{2} .
\end{aligned}
$$

This system can be rewritten as

$$
\begin{aligned}
\Delta \dot{x}_{1} & =\theta_{1} \Delta z_{1}-\gamma_{p} \Delta x_{1} \\
\left(1+B_{x}\right) \Delta \dot{x}_{2} & =k \Delta x_{1}-\gamma_{p}\left(1+B_{x} \frac{\gamma_{x}}{\gamma_{p}}\right) \Delta x_{2} \\
\Delta \dot{z}_{1} & =-\frac{\alpha}{\left(1+B_{x} \frac{\gamma_{x}}{\gamma_{p}}\right)} \Delta z_{1}-\frac{\beta\left(1+B_{x} \frac{\gamma_{x}}{\gamma_{p}}\right)}{\alpha} \Delta z_{2} \\
\Delta \dot{z}_{2} & =\theta_{2} \Delta x_{2}-\frac{\alpha}{\left(1+B_{x} \frac{\gamma_{x}}{\gamma_{p}}\right)} \Delta z_{1}-\frac{\beta\left(1+B_{x} \frac{\gamma_{x}}{\gamma_{p}}\right)}{\alpha} \Delta z_{2} .
\end{aligned}
$$


where $\alpha=\frac{\theta_{1} \theta_{2} k}{\gamma_{p}^{2}}$ and $\beta=\eta \mu$. Taking the Laplace transforms, we have

$$
\begin{aligned}
& \left(s+\gamma_{p}\right) X_{1}=\theta_{1} Z_{1} \\
& \left(1+B_{x}\right)\left(s+\frac{\gamma_{p}+B_{x} \gamma_{x}}{1+B_{x}}\right) X_{2}=k X_{1} \\
& \left(s+\frac{\alpha}{\left(1+B_{x} \frac{\gamma_{x}}{\gamma_{p}}\right)}\right) Z_{1}=-\frac{\beta\left(1+B_{x} \frac{\gamma_{x}}{\gamma_{p}}\right)}{\alpha} Z_{2} \\
& \left(s+\frac{\beta\left(1+B_{x} \frac{\gamma_{x}}{\gamma_{p}}\right)}{\alpha}\right) Z_{2}=\theta_{2} X_{2}-\frac{\alpha}{\left(1+B_{x} \frac{\gamma_{x}}{\gamma_{p}}\right)} Z_{1}
\end{aligned}
$$

where $Z_{1}=\mathcal{L}\left\{\Delta z_{1}\right\}, Z_{2}=\mathcal{L}\left\{\Delta z_{2}\right\}, X_{1}=\mathcal{L}\left\{\Delta x_{1}\right\}, X_{2}=\mathcal{L}\left\{\Delta x_{2}\right\}$ are the Laplace transforms of the time-domain concentration deviations. Substituting, we have

$$
\begin{aligned}
& \left(1+B_{x}\right)\left(s+\gamma_{p}\right)\left(s+\frac{\gamma_{p}+B_{x} \gamma_{x}}{1+B_{x}}\right) X_{2}=\theta_{1} k Z_{1} \\
& \left(s+\frac{\alpha}{\left(1+B_{x} \frac{\gamma_{x}}{\gamma_{p}}\right)}\right)\left(s+\frac{\beta\left(1+B_{x} \frac{\gamma_{x}}{\gamma_{p}}\right)}{\alpha}\right) Z_{1} \\
& =-\frac{\beta\left(1+B_{x} \frac{\gamma_{x}}{\gamma_{p}}\right)}{\alpha}\left(\theta_{2} X_{2}-\frac{\alpha}{\left(1+B_{x} \frac{\gamma_{x}}{\gamma_{p}}\right)} Z_{1}\right) .
\end{aligned}
$$

Rewriting and substituting, we have

$$
\begin{aligned}
& \left(1+B_{x}\right)\left(s+\gamma_{p}\right)\left(s+\frac{\gamma_{p}+B_{x} \gamma_{x}}{1+B_{x}}\right) s\left(s+\frac{\alpha}{\left(1+B_{x} \frac{\gamma_{x}}{\gamma_{p}}\right)}+\frac{\beta\left(1+B_{x} \frac{\gamma_{x}}{\gamma_{p}}\right)}{\alpha}\right) X_{2} \\
& =-\frac{\beta\left(1+B_{x} \frac{\gamma_{x}}{\gamma_{p}}\right)}{\alpha} \theta_{1} \theta_{2} k X_{2} .
\end{aligned}
$$

Simplifying and taking the limit of strong binding for the sequestration process in the antithetic integral feedback

$$
\begin{aligned}
& \left(\beta \gg \frac{\alpha^{2}}{\left(1+B_{x} \frac{\gamma_{x}}{\gamma_{p}}\right)^{2}}, \frac{\alpha \gamma_{p}}{\left(1+B_{x} \frac{\gamma_{x}}{\gamma_{p}}\right)}\right) \text { then } \\
& \qquad\left(1+B_{x}\right)\left(s+\gamma_{p}\right)\left(s+\frac{\gamma_{p}+B_{x} \gamma_{x}}{1+B_{x}}\right) s\left(s+\frac{\beta\left(1+B_{x} \frac{\gamma_{x}}{\gamma_{p}}\right)}{\alpha}\right) X_{2}=-\frac{\beta\left(1+B_{x} \frac{\gamma_{x}}{\gamma_{p}}\right)}{\alpha} \theta_{1} \theta_{2} k X_{2} .
\end{aligned}
$$

Thus we have the characteristic equation

$$
\left(1+B_{x}\right)\left(s+\gamma_{p}\right)\left(s+\frac{\gamma_{p}+B_{x} \gamma_{x}}{1+B_{x}}\right) s\left(s+\frac{\beta\left(1+B_{x} \frac{\gamma_{x}}{\gamma_{p}}\right)}{\alpha}\right)+\beta \gamma_{p}^{2}\left(1+B_{x} \frac{\gamma_{x}}{\gamma_{p}}\right)=0 .
$$

Substituting $s=\gamma_{p} \sigma$, we have

$$
\left(1+B_{x}\right)(1+\sigma)\left(\sigma+\frac{1+B_{x} \frac{\gamma_{x}}{\gamma_{p}}}{1+B_{x}}\right) \sigma\left(\sigma+\frac{\beta\left(1+B_{x} \frac{\gamma_{x}}{\gamma_{p}}\right)}{\gamma_{p} \alpha}\right)=-\frac{\beta}{\gamma_{p}^{2}}\left(1+B_{x} \frac{\gamma_{x}}{\gamma_{p}}\right) .
$$

Using the same argument as that in SI2.1, for the stability boundary with strong binding there is a negative real and complex pair of roots in the region $|\sigma| \ll \frac{\beta\left(1+B_{x} \frac{\gamma_{x}}{\gamma_{y}}\right)}{\gamma_{p}}$, as well as one large negative root. 
To determine the boundary of stability, we next determine the conditions for which the roots are purely imaginary. Substituting $s=i \omega \gamma_{p}$, we have

$$
\left(1+B_{x}\right)(1+i \omega)\left(i \omega+\frac{1+B_{x} \frac{\gamma_{x}}{\gamma_{p}}}{1+B_{x}}\right) i \omega\left(i \omega+\frac{\beta\left(1+B_{x} \frac{\gamma_{x}}{\gamma_{p}}\right)}{\gamma_{p} \alpha}\right)=-\frac{\beta}{\gamma_{p}^{2}}\left(1+B_{x} \frac{\gamma_{x}}{\gamma_{p}}\right)
$$

Taking the strong antithetic binding limit where $|i \omega| \ll \frac{\beta\left(1+B_{x} \frac{\gamma x}{\gamma_{y}}\right)}{\gamma_{p} \alpha}$, we have

$$
(1+i \omega)\left(i \omega+\frac{1+B_{x} \frac{\gamma_{x}}{\gamma_{p}}}{1+B_{x}}\right) i \omega=-\frac{\alpha}{\gamma_{p}\left(1+B_{x}\right)} .
$$

The phase and magnitude constraints are

$$
\begin{aligned}
& \left(1+\omega^{2}\right)^{0.5}\left(\omega^{2}+\left(\frac{1+B_{x} \frac{\gamma_{x}}{\gamma_{p}}}{1+B_{x}}\right)^{2}\right)^{0.5} \omega=\frac{\alpha}{\gamma_{p}\left(1+B_{x}\right)} \\
& \tan ^{-1}(\omega)+\tan ^{-1}\left(\frac{1+B_{x}}{1+B_{x} \frac{\gamma_{x}}{\gamma_{p}}} \omega\right)=\frac{\pi}{2}+2 k \pi
\end{aligned}
$$

for some integer $k$. Solving the phase constraint, we have

$$
\begin{aligned}
& \frac{\omega-1}{1+\omega}=\frac{1-C \omega}{1+C \omega} \\
& C=\frac{1+B_{x}}{1+B_{x} \frac{\gamma_{x}}{\gamma_{p}}} .
\end{aligned}
$$

For this constraint we require

$$
C \omega^{2}=1
$$

which reduces to

$$
\omega^{2}=\frac{1+B_{x} \frac{\gamma_{x}}{\gamma_{p}}}{1+B_{x}}
$$

Substituting into the magnitude equation, we have

$$
\begin{aligned}
& \frac{\alpha}{\gamma_{p}\left(1+B_{x}\right)}=(1+C)^{0.5}\left(C+C^{-2}\right)^{0.5} C^{-0.5} \\
& =(1+C) C .
\end{aligned}
$$

Rearranging, we have the stability condition

$$
\gamma_{p}^{3}>\frac{\theta_{1} \theta_{2} k}{2+\left(1+\frac{\gamma_{x}}{\gamma_{p}}\right) B_{x}} \frac{1+B_{x}}{1+\frac{\gamma_{x}}{\gamma_{p}} B_{x}} .
$$

We can differentiate the right hand side with respect to $B_{x}$ to determine whether increasing $B_{x}$ has a stabilising effect. If we set

$$
f\left(B_{x}\right)=\frac{1+B_{x}}{\left(2+\left(1+\frac{\gamma_{x}}{\gamma_{p}}\right) B_{x}\right)\left(1+\frac{\gamma_{x}}{\gamma_{p}} B_{x}\right)} .
$$

then

$$
\frac{\partial f}{\partial B_{x}}=\frac{1-3 \frac{\gamma_{x}}{\gamma_{p}}-2 \frac{\gamma_{x}}{\gamma_{p}}\left(1+\frac{\gamma_{x}}{\gamma_{p}}\right) B_{x}-\left(1+\frac{\gamma_{x}}{\gamma_{p}}\right) \frac{\gamma_{x}}{\gamma_{p}} B_{x}^{2}}{\left(2+\left(1+\frac{\gamma_{x}}{\gamma_{p}}\right) B_{x}\right)^{2}\left(1+\frac{\gamma_{x}}{\gamma_{p}} B_{x}\right)^{2}}
$$

Thus for small $B_{x}$ then buffering stabilises antithetic integral feedback if $\gamma_{x}>\frac{1}{3} \gamma_{p}$. For large $B_{x}$ then increasing $B_{x}$ improves stability if $\gamma_{x}>0$. 


\section{S12.5. Stability of Antithetic Feedback: Rapid $x_{1}$ Buffering with Degradation}

In this section, we analyse the stabilising effect of buffering at the intermediate species $x_{1}$ on antithetic integral feedback, where the buffering can be degraded. This section uses identical methodology and obtains equivalent results to SI2.4.

Consider the model (S4) with buffering at $x_{1}$ that is degraded

$$
\begin{aligned}
& \dot{x}_{1}=\theta_{1} z_{1}-\gamma_{p} x_{1}-b_{i} x_{1}+b_{w} w_{i} \\
& \dot{x}_{2}=k x_{1}-\gamma_{p} x_{2} \\
& \dot{w}_{i}=b_{i} x_{1}-b_{w} w_{i}-\gamma_{i} w_{x} \\
& \dot{z}_{1}=\mu-\eta z_{1} z_{2} \\
& \dot{z}_{2}=\theta_{2} x_{2}-\eta z_{1} z_{2}
\end{aligned}
$$

where $w_{i}$ is the buffering species of $x_{1}$, and $b_{i}, b_{w}$ are the kinetic rates for the buffering reactions. If we assume rapid buffering such that $w_{i}+x_{1}$ is the slow variable, $w_{i}=B_{i} x_{1}$ and $B_{i}=\frac{b_{i}}{b_{w}+\gamma_{i}}$, and use the methodology from SI2.4 then

$$
\begin{aligned}
\left(1+B_{i}\right) \dot{x}_{1} & =\theta_{1} z_{1}-\left(\gamma_{p}+B_{x} \gamma_{i}\right) x_{1} \\
\dot{x}_{2} & =k x_{1}-\gamma_{p} x_{2} \\
\dot{z}_{1} & =\mu-\eta z_{1} z_{2} \\
\dot{z}_{2} & =\theta_{2} x_{2}-\eta z_{1} z_{2} .
\end{aligned}
$$

\section{SI2.5.a. Steady State Analysis}

We next analyse the steady state. We have

$$
\dot{z}_{1}-\dot{z}_{2}=\mu-\theta_{2} x_{2}
$$

and so the steady state of the output is

$$
x_{2}=\frac{\mu}{\theta_{2}} .
$$

The corresponding steady states of the other species are

$$
\begin{aligned}
& x_{1}=\frac{\gamma_{p}}{k} x_{2}=\frac{\gamma_{p}}{k} \frac{\mu}{\theta_{2}} \\
& z_{1}=\frac{\left(\gamma_{p}+B_{i} \gamma_{i}\right)}{\theta_{1}} x_{1}=\frac{\gamma_{p}^{2} \mu}{\theta_{1} \theta_{2} k}\left(1+B_{i} \frac{\gamma_{i}}{\gamma_{p}}\right)=\frac{\mu}{\alpha}\left(1+B_{i} \frac{\gamma_{i}}{\gamma_{p}}\right) \\
& z_{2}=\frac{1}{\eta} \frac{\mu}{z_{1}}=\frac{\alpha}{\eta\left(1+B_{i} \frac{\gamma_{i}}{\gamma_{p}}\right)}
\end{aligned}
$$

where $\alpha=\frac{\theta_{1} \theta_{2} k}{\gamma_{p}^{2}}$.

\section{S12.5.b. Stability Analysis}

We next study the stability of the system. If we linearise about the steady states, we have

$$
\begin{aligned}
\left(1+B_{i}\right) \Delta \dot{x}_{1} & =\theta_{1} \Delta z_{1}-\gamma_{p}\left(1+B_{i} \frac{\gamma_{i}}{\gamma_{p}}\right) \Delta x_{1} \\
\Delta \dot{x}_{2} & =k \Delta x_{1}-\gamma_{p} \Delta x_{2} \\
\Delta \dot{z}_{1} & =-\eta \bar{z}_{2} \Delta z_{1}-\eta \bar{z}_{1} \Delta z_{2} \\
\Delta \dot{z}_{2} & =\theta_{2} \Delta x_{2}-\eta \bar{z}_{2} \Delta z_{1}-\eta \bar{z}_{1} \Delta z_{2} .
\end{aligned}
$$


This system can be rewritten as

$$
\begin{aligned}
\left(1+B_{i}\right) \Delta \dot{x}_{1} & =\theta_{1} \Delta z_{1}-\gamma_{p}\left(1+B_{i} \frac{\gamma_{i}}{\gamma_{p}}\right) \Delta x_{1} \\
\Delta \dot{x}_{2} & =k \Delta x_{1}-\gamma_{p} \Delta x_{2} \\
\Delta \dot{z}_{1} & =-\frac{\alpha}{\left(1+B_{i} \frac{\gamma_{i}}{\gamma_{p}}\right)} \Delta z_{1}-\frac{\beta\left(1+B_{i} \frac{\gamma_{i}}{\gamma_{p}}\right)}{\alpha} \Delta z_{2} \\
\Delta \dot{z}_{2} & =\theta_{2} \Delta x_{2}-\frac{\alpha}{\left(1+B_{i} \frac{\gamma_{i}}{\gamma_{p}}\right)} \Delta z_{1}-\frac{\beta\left(1+B_{i} \frac{\gamma_{i}}{\gamma_{p}}\right)}{\alpha} \Delta z_{2} .
\end{aligned}
$$

where $\alpha=\frac{\theta_{1} \theta_{2} k}{\gamma_{p}^{2}}$ and $\beta=\eta \mu$. Taking the Laplace transforms, we have

$$
\begin{aligned}
& \left(1+B_{i}\right)\left(s+\frac{\gamma_{p}+B_{i} \gamma_{i}}{1+B_{i}}\right) X_{1}=\theta_{1} Z_{1} \\
& \left(s+\gamma_{p}\right) X_{2}=k X_{1} \\
& \left(s+\frac{\alpha}{\left(1+B_{i} \frac{\gamma_{i}}{\gamma_{p}}\right)}\right) Z_{1}=-\frac{\beta\left(1+B_{i} \frac{\gamma_{i}}{\gamma_{p}}\right)}{\alpha} Z_{2} \\
& \left(s+\frac{\beta\left(1+B_{i} \frac{\gamma_{i}}{\gamma_{p}}\right)}{\alpha}\right) Z_{2}=\theta_{2} X_{2}-\frac{\alpha}{\left(1+B_{i} \frac{\gamma_{i}}{\gamma_{p}}\right)} Z_{1}
\end{aligned}
$$

where $Z_{1}=\mathcal{L}\left\{\Delta z_{1}\right\}, Z_{2}=\mathcal{L}\left\{\Delta z_{2}\right\}, X_{1}=\mathcal{L}\left\{\Delta x_{1}\right\}, X_{2}=\mathcal{L}\left\{\Delta x_{2}\right\}$ are the Laplace transforms of the time-domain concentration deviations. Substituting, we have

$$
\begin{aligned}
& \left(1+B_{i}\right)\left(s+\gamma_{p}\right)\left(s+\frac{\gamma_{p}+B_{i} \gamma_{i}}{1+B_{i}}\right) X_{2}=\theta_{1} k Z_{1} \\
& \left(s+\frac{\alpha}{\left(1+B_{i} \frac{\gamma_{i}}{\gamma_{p}}\right)}\right)\left(s+\frac{\beta\left(1+B_{i} \frac{\gamma_{i}}{\gamma_{p}}\right)}{\alpha}\right) Z_{1}=-\frac{\beta\left(1+B_{i} \frac{\gamma_{i}}{\gamma_{p}}\right)}{\alpha}\left(\theta_{2} X_{2}-\frac{\alpha}{\left(1+B_{i} \frac{\gamma_{i}}{\gamma_{p}}\right)} Z_{1}\right)
\end{aligned}
$$

Rewriting and substituting, we have

$$
\left(1+B_{i}\right)\left(s+\gamma_{p}\right)\left(s+\frac{\gamma_{p}+B_{i} \gamma_{i}}{1+B_{i}}\right) s\left(s+\frac{\alpha}{\left(1+B_{i} \frac{\gamma_{i}}{\gamma_{p}}\right)}+\frac{\beta\left(1+B_{i} \frac{\gamma_{i}}{\gamma_{p}}\right)}{\alpha}\right) X_{2}=-\frac{\beta\left(1+B_{i} \frac{\gamma_{i}}{\gamma_{p}}\right)}{\alpha} \theta_{1} \theta_{2} k X_{2}
$$

The above equation is equivalent to $x_{2}$ buffering (see SI2.5), and so for strong integral binding we have the stability condition

$$
\gamma_{p}^{3}>\frac{\theta_{1} \theta_{2} k}{2+\left(1+\frac{\gamma_{i}}{\gamma_{p}}\right) B_{i}} \frac{1+B_{i}}{1+\frac{\gamma_{i}}{\gamma_{p}} B_{i}}
$$

which is equivalent to the case of $x_{2}$ buffering. 


\section{SI3. BUFFERING CAN ENABLE NEAR-PERFECT ADAPTATION DESPITE LEAKY INTEGRATION}

\section{S13.1. Rapid $x_{2}$ Buffering with Degradation can Enable Near-Perfect Adaptation}

In this section, we analyse the ability of buffering at $x_{2}$ to enable near perfect adaptation by stabilising antithetic integral feedback. Consider the model (S5) with buffering at $x_{2}$ that is degraded

$$
\begin{aligned}
\dot{x}_{1} & =\theta_{1} z_{1}-\gamma_{p} x_{1} \\
\dot{x}_{2} & =k x_{1}-\gamma_{p} x_{2}-b_{x} x_{2}+b_{w} w_{x} \\
\dot{w}_{x} & =b_{x} x_{2}-b_{w} w_{x}-\gamma_{x} w_{x} \\
\dot{z}_{1} & =\mu-\eta z_{1} z_{2}-\gamma_{c} z_{1} \\
\dot{z}_{2} & =\theta_{2} x_{2}-\eta z_{1} z_{2}-\gamma_{c} z_{2}
\end{aligned}
$$

where $w_{x}$ is the buffering species of $x_{2}$, and $b_{x}, b_{w}$ are the kinetic rates for the buffering reactions. Assuming that the buffer rapidly reaches equilibrium then using the methodology from SI1 and SI2.1, we have the reduced model

$$
\begin{aligned}
\dot{x}_{1} & =\theta_{1} z_{1}-\gamma_{p} x_{1} \\
\left(1+B_{x}\right) \dot{x}_{2} & =k x_{1}-\left(\gamma_{p}+B_{x} \gamma_{x}\right) x_{2} \\
\dot{z}_{1} & =\mu-\eta z_{1} z_{2}-\gamma_{c} z_{1} \\
\dot{z}_{2} & =\theta_{2} x_{2}-\eta z_{1} z_{2}-\gamma_{c} z_{2}
\end{aligned}
$$

where

$$
\begin{aligned}
B_{x} & =\frac{b_{x}}{b_{w}+\gamma_{c}} \\
w_{x} & =B_{x} x_{2} .
\end{aligned}
$$

\section{SI3.1.a. Steady State Analysis}

We next analyse the steady state. We have

$$
\dot{z}_{1}-\dot{z}_{2}=\mu-\theta_{2} x_{2}-\gamma_{c} z_{1}+\gamma_{c} z_{2}
$$

and so the steady state of the output is

$$
x_{2}=\frac{\mu}{\theta_{2}}-\frac{\gamma_{c}}{\theta_{2}} z_{1}+\frac{\gamma_{c}}{\theta_{2}} z_{2}
$$

We also have the steady state

$$
z_{2}=\frac{1}{\eta}\left(\frac{\mu}{z_{1}}-\gamma_{c}\right)
$$

and so

$$
x_{2}=\frac{\mu}{\theta_{2}}-\frac{\gamma_{c}^{2}}{\theta_{2} \eta}-\frac{\gamma_{c}}{\theta_{2}} z_{1}+\frac{\gamma_{c} \mu}{\theta_{2} \eta} \frac{1}{z_{1}} .
$$

The other species have the steady states

$$
\begin{aligned}
& x_{1}=\frac{\gamma_{p}}{k}\left(1+B_{x} \frac{\gamma_{x}}{\gamma_{p}}\right) x_{2} \\
& z_{1}=\frac{\gamma_{p}}{\theta_{1}} x_{1}=\frac{\gamma_{p}^{2}}{\theta_{1} k}\left(1+B_{x} \frac{\gamma_{x}}{\gamma_{p}}\right) x_{2}=\frac{\theta_{2}}{\alpha}\left(1+B_{x} \frac{\gamma_{x}}{\gamma_{p}}\right) x_{2}
\end{aligned}
$$


where $\alpha=\frac{\theta_{1} \theta_{2} k}{\gamma_{p}^{2}}$. Substituting, we have

$$
\left(1+\frac{\gamma_{c}}{\alpha}\left(1+B_{x} \frac{\gamma_{x}}{\gamma_{p}}\right)\right) x_{2}^{2}=\left(\frac{\mu}{\theta_{2}}-\frac{\gamma_{c}^{2}}{\theta_{2} \eta}\right) x_{2}+\frac{\gamma_{c} \mu \alpha}{\theta_{2}^{2} \eta\left(1+B_{x} \frac{\gamma_{x}}{\gamma_{p}}\right)}
$$

We assume strong binding of the sequestration mechanism in antithetic integral feedback ( $\eta$ large), which for steady state is the condition

$$
\beta \gg \frac{\gamma_{c}^{2}}{\mu}, \frac{\gamma_{c} \mu \alpha}{\theta_{2}^{2}\left(1+B_{x} \frac{\gamma_{x}}{\gamma_{p}}\right)}
$$

With this assumption, we have

$$
\left(1+\frac{\gamma_{c}}{\alpha}\left(1+B_{x} \frac{\gamma_{x}}{\gamma_{p}}\right)\right) x_{2}^{2}=\frac{\mu}{\theta_{2}} x_{2}
$$

We ignore the zero solution, which corresponds to a negative solution in (S25), and so the steady state concentrations are

$$
\begin{gathered}
x_{2}=\frac{\mu}{\theta_{2}} \frac{1}{1+\frac{\gamma_{c}}{\alpha}\left(1+B_{x} \frac{\gamma_{x}}{\gamma_{p}}\right)} \\
x_{1}=\frac{\gamma_{p}}{k} \frac{\mu}{\theta_{2}} \frac{\left(1+B_{x} \frac{\gamma_{x}}{\gamma_{p}}\right)}{1+\frac{\gamma_{c}}{\alpha}\left(1+B_{x} \frac{\gamma_{x}}{\gamma_{p}}\right)}, \quad z_{1}=\frac{\mu\left(1+B_{x} \frac{\gamma_{x}}{\gamma_{p}}\right)}{\alpha+\gamma_{c}\left(1+B_{x} \frac{\gamma_{x}}{\gamma_{p}}\right)}, \quad z_{2}=\frac{\alpha}{\eta\left(1+B_{x} \frac{\gamma_{x}}{\gamma_{p}}\right)} .
\end{gathered}
$$

The steady state error of $x_{2}$ is

$$
\frac{x_{2 n}-x_{2}}{x_{2 n}}=\frac{1}{1+\Omega_{x}}, \quad \Omega_{x}=\frac{\alpha}{\gamma_{c}\left(1+B_{x} \frac{\gamma_{x}}{\gamma_{p}}\right)}
$$

\section{S13.1.b. Stability Analysis}

We next study the stability of the system. If we linearise (S24) about the steady state, we have

$$
\begin{aligned}
\Delta \dot{x}_{1} & =\theta_{1} \Delta z_{1}-\gamma_{p} \Delta x_{1} \\
\left(1+B_{x}\right) \Delta \dot{x}_{2} & =k \Delta x_{1}-\gamma_{p}\left(1+B_{x} \frac{\gamma_{x}}{\gamma_{p}}\right) \Delta x_{2} \\
\Delta \dot{z}_{1} & =-\eta \bar{z}_{2} \Delta z_{1}-\eta \bar{z}_{1} \Delta z_{2}-\gamma_{c} \Delta z_{1} \\
\Delta \dot{z}_{2} & =\theta_{2} \Delta x_{2}-\eta \bar{z}_{2} \Delta z_{1}-\eta \bar{z}_{1} \Delta z_{2}-\gamma_{c} \Delta z_{2} .
\end{aligned}
$$

This system can be rewritten as

$$
\begin{aligned}
\Delta \dot{x}_{1} & =\theta_{1} \Delta z_{1}-\gamma_{p} \Delta x_{1} \\
\left(1+B_{x}\right) \Delta \dot{x}_{2} & =k \Delta x_{1}-\gamma_{p}\left(1+B_{x} \frac{\gamma_{x}}{\gamma_{p}}\right) \Delta x_{2} \\
\Delta \dot{z}_{1} & =-\left(\frac{\alpha}{\left(1+B_{x} \frac{\gamma_{x}}{\gamma_{p}}\right)}+\gamma_{c}\right) \Delta z_{1}-\frac{\beta\left(1+B_{x} \frac{\gamma_{x}}{\gamma_{p}}\right)}{\alpha+\gamma_{c}\left(1+B_{x} \frac{\gamma_{x}}{\gamma_{p}}\right)} \Delta z_{2} \\
\Delta \dot{z}_{2} & =\theta_{2} \Delta x_{2}-\frac{\alpha}{\left(1+B_{x} \frac{\gamma_{x}}{\gamma_{p}}\right)} \Delta z_{1}-\left(\frac{\beta\left(1+B_{x} \frac{\gamma_{x}}{\gamma_{p}}\right)}{\alpha+\gamma_{c}\left(1+B_{x} \frac{\gamma_{x}}{\gamma_{p}}\right)}+\gamma_{c}\right) \Delta z_{2}
\end{aligned}
$$


where $\alpha=\frac{\theta_{1} \theta_{2} k}{\gamma_{p}^{2}}$ and $\beta=\eta \mu$. Taking the Laplace transforms, we have

$$
\begin{aligned}
& \left(s+\gamma_{p}\right) X_{1}=\theta_{1} Z_{1} \\
& \left(1+B_{x}\right)\left(s+\frac{\gamma_{p}+B_{x} \gamma_{x}}{1+B_{x}}\right) X_{2}=k X_{1} \\
& \left(s+\frac{\alpha}{\left(1+B_{x} \frac{\gamma_{x}}{\gamma_{p}}\right)}+\gamma_{c}\right) Z_{1}=-\frac{\beta\left(1+B_{x} \frac{\gamma_{x}}{\gamma_{p}}\right)}{\alpha+\gamma_{c}\left(1+B_{x} \frac{\gamma_{x}}{\gamma_{p}}\right)} Z_{2} \\
& \left(s+\frac{\beta\left(1+B_{x} \frac{\gamma_{x}}{\gamma_{p}}\right)}{\alpha+\gamma_{c}\left(1+B_{x} \frac{\gamma_{x}}{\gamma_{p}}\right)}+\gamma_{c}\right) Z_{2}=\theta_{2} X_{2}-\frac{\alpha}{\left(1+B_{x} \frac{\gamma_{x}}{\gamma_{p}}\right)} Z_{1}
\end{aligned}
$$

where $X_{1}, X_{1}, Z_{1}$ and $Z_{2}$ are the Laplace transforms of $\Delta x_{1}, \Delta x_{2}, \Delta z_{1}$ and $\Delta z_{2}$. Substituting, we have

$$
\begin{aligned}
& \left(1+B_{x}\right)\left(s+\gamma_{p}\right)\left(s+\frac{\gamma_{p}+B_{x} \gamma_{x}}{1+B_{x}}\right) X_{2}=\theta_{1} k Z_{1} \\
& \left(s+\frac{\alpha}{\left(1+B_{x} \frac{\gamma_{x}}{\gamma_{p}}\right)}+\gamma_{c}\right)\left(s+\frac{\beta\left(1+B_{x} \frac{\gamma_{x}}{\gamma_{p}}\right)}{\alpha+\gamma_{c}\left(1+B_{x} \frac{\gamma_{x}}{\gamma_{p}}\right)}+\gamma_{c}\right) Z_{1} \\
& =-\frac{\beta\left(1+B_{x} \frac{\gamma_{x}}{\gamma_{p}}\right)}{\alpha+\gamma_{c}\left(1+B_{x} \frac{\gamma_{x}}{\gamma_{p}}\right)}\left(\theta_{2} X_{2}-\frac{\alpha}{\left(1+B_{x} \frac{\gamma_{x}}{\gamma_{p}}\right)} Z_{1}\right)
\end{aligned}
$$

Rewriting and substituting, we have

$$
\begin{aligned}
& \left(1+B_{x}\right)\left(s+\gamma_{p}\right)\left(s+\frac{\gamma_{p}+B_{x} \gamma_{x}}{1+B_{x}}\right)\left[\left(s+\gamma_{c}\right)\left(s+\frac{\alpha}{\left(1+B_{x} \frac{\gamma_{x}}{\gamma_{p}}\right)}+\frac{\beta\left(1+B_{x} \frac{\gamma_{x}}{\gamma_{p}}\right)}{\alpha+\gamma_{c}\left(1+B_{x} \frac{\gamma_{x}}{\gamma_{p}}\right)}+\gamma_{c}\right)\right] X_{2} \\
& =-\frac{\beta\left(1+B_{x} \frac{\gamma_{x}}{\gamma_{p}}\right)}{\alpha+\gamma_{c}\left(1+B_{x} \frac{\gamma_{x}}{\gamma_{p}}\right)} \theta_{1} \theta_{2} k X_{2} .
\end{aligned}
$$

Simplifying and taking the limit of strong binding of the sequestration mechanism in antithetic integral feedback

$$
\beta \gg \frac{\left(\alpha+\gamma_{c}\left(1+B_{x} \frac{\gamma_{x}}{\gamma_{p}}\right)\right)^{2}}{\left(1+B_{x} \frac{\gamma_{x}}{\gamma_{p}}\right)^{2}}, \frac{\left(\alpha+\gamma_{c}\left(1+B_{x} \frac{\gamma_{x}}{\gamma_{p}}\right)\right) \gamma_{p}}{\left(1+B_{x} \frac{\gamma_{x}}{\gamma_{p}}\right)}
$$

then

$$
\begin{aligned}
& \left(1+B_{x}\right)\left(s+\gamma_{p}\right)\left(s+\frac{\gamma_{p}+B_{x} \gamma_{x}}{1+B_{x}}\right)\left(s+\gamma_{c}\right)\left(s+\frac{\beta\left(1+B_{x} \frac{\gamma_{x}}{\gamma_{p}}\right)}{\alpha+\gamma_{c}\left(1+B_{x} \frac{\gamma_{x}}{\gamma_{p}}\right)}\right) X_{2} \\
& =-\frac{\beta\left(1+B_{x} \frac{\gamma_{x}}{\gamma_{p}}\right)}{\alpha+\gamma_{c}\left(1+B_{x} \frac{\gamma_{x}}{\gamma_{p}}\right)} \theta_{1} \theta_{2} k X_{2} .
\end{aligned}
$$

Thus we have the characteristic equation

$$
\left(1+B_{x}\right)\left(s+\gamma_{p}\right)\left(s+\frac{\gamma_{p}+B_{x} \gamma_{x}}{1+B_{x}}\right)\left(s+\gamma_{c}\right)\left(s+\frac{\beta\left(1+B_{x} \frac{\gamma_{x}}{\gamma_{p}}\right)}{\alpha+\gamma_{c}\left(1+B_{x} \frac{\gamma_{x}}{\gamma_{p}}\right)}\right)+\beta \gamma_{p}^{2} \frac{\alpha\left(1+B_{x} \frac{\gamma_{x}}{\gamma_{p}}\right)}{\alpha+\gamma_{c}\left(1+B_{x} \frac{\gamma_{x}}{\gamma_{p}}\right)}=0 .
$$


Substituting $s=\gamma_{p} \sigma$, we have

$$
\left(1+B_{x}\right)(1+\sigma)\left(\sigma+\frac{1+B_{x} \frac{\gamma_{x}}{\gamma_{p}}}{1+B_{x}}\right)\left(\sigma+\frac{\gamma_{c}}{\gamma_{p}}\right)\left(\sigma+\frac{\beta\left(1+B_{x} \frac{\gamma_{x}}{\gamma_{p}}\right)}{\gamma_{p}\left(\alpha+\gamma_{c}\left(1+B_{x} \frac{\gamma_{x}}{\gamma_{p}}\right)\right)}\right)=-\frac{\beta}{\gamma_{p}^{2}} \frac{\alpha\left(1+B_{x} \frac{\gamma_{x}}{\gamma_{p}}\right)}{\alpha+\gamma_{c}\left(1+B_{x} \frac{\gamma_{x}}{\gamma_{p}}\right)} .
$$

Using the same argument as that used in SI2.1, for the stability boundary with strong binding there is a negative real and complex pair of roots in the region $|\sigma| \ll \frac{\beta\left(1+B_{x} \frac{\gamma x}{\gamma p}\right)}{\gamma_{p}\left(\alpha+\gamma_{c}\left(1+B_{x} \frac{\gamma x}{\gamma p}\right)\right)}$, as well as one large negative root.

To determine the boundary of stability, we next determine the conditions for which the roots are purely imaginary. Substituting $s=i \omega \gamma_{p}$, we have

$$
\left(1+B_{x}\right)(1+i \omega)\left(i \omega+\frac{1+B_{x} \frac{\gamma_{x}}{\gamma_{p}}}{1+B_{x}}\right)\left(i \omega+\frac{\gamma_{c}}{\gamma_{p}}\right)\left(i \omega+\frac{\beta\left(1+B_{x} \frac{\gamma_{x}}{\gamma_{p}}\right)}{\gamma_{p}\left(\alpha+\gamma_{c}\left(1+B_{x} \frac{\gamma_{x}}{\gamma_{p}}\right)\right)}\right)=-\frac{\beta}{\gamma_{p}^{2}} \frac{\alpha\left(1+B_{x} \frac{\gamma_{x}}{\gamma_{p}}\right)}{\alpha+\gamma_{c}\left(1+B_{x} \frac{\gamma_{x}}{\gamma_{p}}\right)} .
$$

Taking the strong antithetic binding limit where $|i \omega| \ll \frac{\beta\left(1+B_{x} \frac{\gamma x}{\gamma p}\right)}{\gamma_{p}\left(\alpha+\gamma_{c}\left(1+B_{x} \frac{\gamma x}{\gamma p}\right)\right)}$, we have

$$
(1+i \omega)\left(i \omega+\frac{1+B_{x} \frac{\gamma_{x}}{\gamma_{p}}}{1+B_{x}}\right)\left(i \omega+\frac{\gamma_{c}}{\gamma_{p}}\right)=-\frac{\alpha}{\gamma_{p}\left(1+B_{x}\right)}
$$

The phase and magnitude constraints are

$$
\begin{aligned}
& \left(1+\omega^{2}\right)^{0.5}\left(\omega^{2}+\left(\frac{1+B_{x} \frac{\gamma_{x}}{\gamma_{p}}}{1+B_{x}}\right)^{2}\right)^{0.5}\left(\omega^{2}+\frac{\gamma_{c}^{2}}{\gamma_{p}^{2}}\right)^{0.5}=\frac{\alpha}{\gamma_{p}\left(1+B_{x}\right)} \\
& \tan ^{-1}(\omega)+\tan ^{-1}\left(\frac{1+B_{x}}{1+B_{x} \frac{\gamma_{x}}{\gamma_{p}}} \omega\right)+\tan ^{-1}\left(\frac{\gamma_{p}}{\gamma_{c}} \omega\right)=\pi+2 k \pi
\end{aligned}
$$

for some integer $k$. Solving the phase constraint, we have

$$
\begin{aligned}
& \tan ^{-1}\left(\frac{\left(1+A^{-1}\right) \omega+\frac{\gamma_{p}}{\gamma_{c}} \omega\left(1-A^{-1} \omega^{2}\right)}{1-\left(A^{-1}+\left(1+A^{-1}\right) \frac{\gamma_{p}}{\gamma_{c}}\right) \omega^{2}}\right)=\pi+2 k \pi \\
& A=\frac{1+B_{x} \frac{\gamma_{x}}{\gamma_{p}}}{1+B_{x}} .
\end{aligned}
$$

For this we require

$$
\left(1+A^{-1}\right) \omega+\frac{\gamma_{p}}{\gamma_{c}} \omega\left(1-A^{-1} \omega^{2}\right)=0
$$

which reduces to

$$
\omega^{2}=\frac{\gamma_{c}}{\gamma_{p}}(1+A)+A
$$

Substituting into the magnitude equation, we have

$$
\begin{aligned}
& \left(\left(1+\frac{\gamma_{c}}{\gamma_{p}}\right)(1+A)\right)^{0.5}\left(\left(\frac{\gamma_{c}}{\gamma_{p}}+A\right)(1+A)\right)^{0.5}\left(\left(\frac{\gamma_{c}}{\gamma_{p}}+1\right)\left(\frac{\gamma_{c}}{\gamma_{p}}+A\right)\right)^{0.5} \\
& =\left(1+\frac{\gamma_{c}}{\gamma_{p}}\right)(1+A)\left(\frac{\gamma_{c}}{\gamma_{p}}+A\right) \\
& =\frac{\alpha}{\gamma_{p}\left(1+B_{x}\right)} .
\end{aligned}
$$


Simplifying, we have

$$
\frac{\alpha}{\gamma_{p}\left(1+B_{x}\right)}=\left(1+\frac{\gamma_{c}}{\gamma_{p}}\right)(1+A)\left(\frac{\gamma_{c}}{\gamma_{p}}+A\right)
$$

Multiplying by $A^{-1} \frac{\gamma_{p}}{\gamma_{c}}$, we have

$$
\begin{aligned}
\frac{\alpha}{\gamma_{c}\left(1+B_{x} \frac{\gamma_{x}}{\gamma_{p}}\right)} & =A^{-1} \frac{\gamma_{p}}{\gamma_{c}}\left(1+\frac{\gamma_{c}}{\gamma_{p}}\right)(1+A)\left(\frac{\gamma_{c}}{\gamma_{p}}+A\right) \\
A & =\frac{1+B_{x} \frac{\gamma_{x}}{\gamma_{p}}}{1+B_{x}} .
\end{aligned}
$$

and so the stability condition is

$$
\begin{aligned}
& \Omega_{x}<\left(1+\frac{\gamma_{c}}{\gamma_{p}}\right)(1+A)\left(\frac{\gamma_{p}}{\gamma_{c}}+A^{-1}\right) \\
& A=\frac{1+B_{x} \frac{\gamma_{x}}{\gamma_{p}}}{1+B_{x}}
\end{aligned}
$$

where

$$
\Omega_{x}=\frac{\alpha}{\gamma_{c}\left(1+B_{x} \frac{\gamma_{x}}{\gamma_{p}}\right)} .
$$

From earlier, we know that the steady state error of $x_{2}$ is

$$
\frac{x_{2 n}-x_{2}}{x_{2 n}}=\frac{1}{1+\Omega_{x}}
$$

\section{SI3.2. Non-Rapid $x_{2}$ Buffering can enable Near Perfect Adaptation}

In this section, we analyse the ability of non-rapid buffering at $z_{1}$ to enable near perfect adaptation by stabilising antithetic integral feedback. We use the model

$$
\begin{aligned}
& \dot{x}_{1}=\theta_{1} z_{1}-\gamma_{p} x_{1} \\
& \dot{x}_{2}=k x_{1}-\gamma_{p} x_{2}-b_{x} x_{2}+b_{w} w \\
& \dot{z}_{1}=\mu-\eta z_{1} z_{2}-\gamma_{c} z_{1} \\
& \dot{z}_{2}=\theta_{2} x_{2}-\eta z_{1} z_{2}-\gamma_{c} z_{2} \\
& \dot{w}=b_{x} x_{2}-b_{w} w-\gamma_{c} w .
\end{aligned}
$$

where the buffer $w$ is not assumed to rapidly reach equilbrium. The steady state for (S26) is identical to the rapid case in SI3.1 if we replace $\gamma_{x}$ by $\gamma_{c}$. The linearisation is

$$
\begin{aligned}
\Delta \dot{x}_{1} & =\theta_{1} \Delta z_{1}-\gamma_{p} \Delta x_{1} \\
\Delta \dot{x}_{2} & =k \Delta x_{1}-\gamma_{p} \Delta x_{2}-b_{x} \Delta x_{2}+b_{w} \Delta w \\
\Delta \dot{z}_{1} & =-\eta \bar{z}_{2} \Delta z_{1}-\eta \bar{z}_{1} \Delta z_{2}-\gamma_{c} \Delta z_{1} \\
\Delta \dot{z}_{2} & =\theta_{2} \Delta x_{2}-\eta \bar{z}_{2} \Delta z_{1}-\eta \bar{z}_{1} \Delta z_{2}-\gamma_{c} \Delta z_{2} \\
\Delta \dot{w} & =b_{x} \Delta x_{2}-\left(b_{w}+\gamma_{c}\right) \Delta w .
\end{aligned}
$$


From SI3.1, the steady states are

$$
\begin{aligned}
& z_{1}=\frac{\gamma_{p}}{\theta_{1}} x_{1}=\frac{\gamma_{p}^{2}}{\theta_{1} k}\left(1+B_{x} \frac{\gamma_{c}}{\gamma_{p}}\right) x_{2}=\frac{\theta_{2}}{\alpha}\left(1+B_{x} \frac{\gamma_{c}}{\gamma_{p}}\right) x_{2} \\
& z_{2}=\frac{1}{\eta}\left(\frac{\mu}{z_{1}}-\gamma_{c}\right) \\
& x_{2}=\frac{\mu}{\theta_{2}} \frac{1}{1+\frac{\gamma_{c}}{\alpha}\left(1+B_{x} \frac{\gamma_{c}}{\gamma_{p}}\right)}
\end{aligned}
$$

where

$$
\alpha=\frac{\theta_{1} \theta_{2} k}{\gamma_{p}^{2}}, \quad \beta=\eta \mu, \quad B_{x}=\frac{b_{x}}{b_{w}+\gamma_{c}}
$$

This system can be rewritten

$$
\begin{aligned}
\Delta \dot{x}_{1} & =\theta_{1} \Delta z_{1}-\gamma_{p} \Delta x_{1} \\
\Delta \dot{x}_{2} & =k \Delta x_{1}-\gamma_{p} \Delta x_{2}-b_{x} \Delta x_{2}+b_{w} \Delta w \\
\Delta \dot{w} & =b_{x} \Delta x_{2}-\left(b_{w}+\gamma_{c}\right) \Delta w \\
\Delta \dot{z}_{1} & =-\left(\frac{\alpha}{\left(1+B_{x} \frac{\gamma_{c}}{\gamma_{p}}\right)}+\gamma_{c}\right) \Delta z_{1}-\frac{\beta\left(1+B_{x} \frac{\gamma_{c}}{\gamma_{p}}\right)}{\alpha+\gamma_{c}\left(1+B_{x} \frac{\gamma_{c}}{\gamma_{p}}\right)} \Delta z_{2} \\
\Delta \dot{z}_{2} & =\theta_{2} \Delta x_{2}-\frac{\alpha}{\left(1+B_{x} \frac{\gamma_{c}}{\gamma_{p}}\right)} \Delta z_{1}-\left(\frac{\beta\left(1+B_{x} \frac{\gamma_{c}}{\gamma_{p}}\right)}{\alpha+\gamma_{c}\left(1+B_{x} \frac{\gamma_{c}}{\gamma_{p}}\right)}+\gamma_{c}\right) \Delta z_{2} .
\end{aligned}
$$

Taking the Laplace transform of $\Delta \dot{w}=b_{x} \Delta x_{2}-\left(b_{w}+\gamma_{c}\right) \Delta w$, we have

$$
W=\frac{b_{x}}{s+b_{w}+\gamma_{c}} X_{2}
$$

where $W$ and $Z_{1}$ are the Laplace transforms of $w$ and $x_{2}$. We have

$$
\begin{aligned}
-b_{x} X_{2}+b_{w} W & =-b_{x} X_{2}+b_{w} \frac{b_{x}}{s+b_{w}+\gamma_{c}} X_{2} \\
& =-B_{x} \frac{s+\gamma_{c}}{1+\frac{s}{b_{w}+\gamma_{c}}} X_{2} \\
& =-C_{b}(s) X_{2}
\end{aligned}
$$

where

$$
C_{b}=B_{x} \frac{s+\gamma_{c}}{1+\frac{s}{\hat{b}_{w}}}, \quad \hat{b}_{w}=b_{w}+\gamma_{c}
$$

Taking the Laplace transforms, we have

$$
\begin{aligned}
& \left(s+\gamma_{p}\right) X_{1}=\theta_{1} Z_{1} \\
& \left(s+\gamma_{p}+C_{b}\right) X_{2}=k X_{1} \\
& \left(s+\frac{\alpha}{\left(1+B_{x} \frac{\gamma_{c}}{\gamma_{p}}\right)}+\gamma_{c}\right) Z_{1}=-\frac{\beta\left(1+B_{x} \frac{\gamma_{c}}{\gamma_{p}}\right)}{\alpha+\gamma_{c}\left(1+B_{x} \frac{\gamma_{c}}{\gamma_{p}}\right)} Z_{2} \\
& \left(s+\frac{\beta\left(1+B_{x} \frac{\gamma_{c}}{\gamma_{p}}\right)}{\alpha+\gamma_{c}\left(1+B_{x} \frac{\gamma_{c}}{\gamma_{p}}\right)}+\gamma_{c}\right) Z_{2}=\theta_{2} X_{2}-\frac{\alpha}{\left(1+B_{x} \frac{\gamma_{c}}{\gamma_{p}}\right)} Z_{1}
\end{aligned}
$$


where $X_{1}, X_{1}, Z_{1}$ and $Z_{2}$ are the Laplace transforms of $\Delta x_{1}, \Delta x_{2}, \Delta z_{1}$ and $\Delta z_{2}$. Substituting, we have

$$
\begin{aligned}
& \left(s+\gamma_{p}\right)\left(s+\gamma_{p}+C_{b}\right) X_{2}=\theta_{1} k Z_{1} \\
& \left(s+\frac{\alpha}{\left(1+B_{x} \frac{\gamma_{c}}{\gamma_{p}}\right)}+\gamma_{c}\right)\left(s+\frac{\beta\left(1+B_{x} \frac{\gamma_{c}}{\gamma_{p}}\right)}{\alpha+\gamma_{c}\left(1+B_{x} \frac{\gamma_{c}}{\gamma_{p}}\right)}+\gamma_{c}\right) Z_{1} \\
& =-\frac{\beta\left(1+B_{x} \frac{\gamma_{c}}{\gamma_{p}}\right)}{\alpha+\gamma_{c}\left(1+B_{x} \frac{\gamma_{c}}{\gamma_{p}}\right)}\left(\theta_{2} X_{2}-\frac{\alpha}{\left(1+B_{x} \frac{\gamma_{c}}{\gamma_{p}}\right)} Z_{1}\right) .
\end{aligned}
$$

Rewriting and substituting, we have

$$
\left(s+\gamma_{p}\right)\left(s+\gamma_{p}+C_{b}\right)\left(s+\gamma_{c}\right)\left(s+\frac{\alpha}{\left(1+B_{x} \frac{\gamma_{c}}{\gamma_{p}}\right)}+\frac{\beta\left(1+B_{x} \frac{\gamma_{c}}{\gamma_{p}}\right)}{\alpha+\gamma_{c}\left(1+B_{x} \frac{\gamma_{c}}{\gamma_{p}}\right)}+\gamma_{c}\right) X_{2}=-\beta \gamma_{p}^{2} \frac{\alpha\left(1+B_{x} \frac{\gamma_{c}}{\gamma_{p}}\right)}{\alpha+\gamma_{c}\left(1+B_{x} \frac{\gamma_{c}}{\gamma_{p}}\right)} X_{2} .
$$

Simplifying and taking the limit of strong binding of the sequestration mechanism in antithetic integral feedback

$$
\beta \gg \frac{\left(\alpha+\gamma_{c}\left(1+B_{x} \frac{\gamma_{c}}{\gamma_{p}}\right)\right)^{2}}{\left(1+B_{x} \frac{\gamma_{c}}{\gamma_{p}}\right)^{2}}, \frac{\left(\alpha+\gamma_{c}\left(1+B_{x} \frac{\gamma_{c}}{\gamma_{p}}\right)\right) \gamma_{p}}{\left(1+B_{x} \frac{\gamma_{c}}{\gamma_{p}}\right)}
$$

then

$$
\left(s+\gamma_{p}\right)\left(s+\gamma_{p}+C_{b}\right)\left(s+\gamma_{c}\right)\left(s+\frac{\beta\left(1+B_{x} \frac{\gamma_{c}}{\gamma_{p}}\right)}{\alpha+\gamma_{c}\left(1+B_{x} \frac{\gamma_{c}}{\gamma_{p}}\right)}\right) X_{2}=-\beta \gamma_{p}^{2} \frac{\alpha\left(1+B_{x} \frac{\gamma_{c}}{\gamma_{p}}\right)}{\alpha+\gamma_{c}\left(1+B_{x} \frac{\gamma_{c}}{\gamma_{p}}\right)} X_{2} .
$$

Rewriting $C_{b}$, we have

$$
\left(s+\gamma_{p}\right)\left(s+\gamma_{p}+B_{x} \frac{s+\gamma_{c}}{1+\frac{s}{\hat{b}_{w}}}\right)\left(s+\gamma_{c}\right)\left(s+\frac{\beta\left(1+B_{x} \frac{\gamma_{c}}{\gamma_{p}}\right)}{\alpha+\gamma_{c}\left(1+B_{x} \frac{\gamma_{c}}{\gamma_{p}}\right)}\right) X_{2}=-\beta \gamma_{p}^{2} \frac{\alpha\left(1+B_{x} \frac{\gamma_{c}}{\gamma_{p}}\right)}{\alpha+\gamma_{c}\left(1+B_{x} \frac{\gamma_{c}}{\gamma_{p}}\right)} X_{2} .
$$

and so we have the characteristic equation

$$
\begin{aligned}
& \left(s+\gamma_{p}\right)\left(\left(s+\gamma_{p}\right)\left(1+\frac{s}{\hat{b}_{w}}\right)+B_{x}\left(s+\gamma_{c}\right)\right)\left(s+\gamma_{c}\right)\left(s+\frac{\beta\left(1+B_{x} \frac{\gamma_{c}}{\gamma_{p}}\right)}{\alpha+\gamma_{c}\left(1+B_{x} \frac{\gamma_{c}}{\gamma_{p}}\right)}\right) \\
& =-\beta \gamma_{p}^{2} \frac{\alpha\left(1+B_{x} \frac{\gamma_{c}}{\gamma_{p}}\right)}{\alpha+\gamma_{c}\left(1+B_{x} \frac{\gamma_{c}}{\gamma_{p}}\right)}\left(1+\frac{s}{\hat{b}_{w}}\right) .
\end{aligned}
$$

Substituting $s=i \omega \gamma_{p}$, we have

$$
\begin{aligned}
& (1+i \omega)\left(1+B_{x} \frac{\gamma_{c}}{\gamma_{p}}-\frac{\gamma_{p}}{\hat{b}_{w}} \omega^{2}+i \omega\left(1+B_{x}+\frac{\gamma_{p}}{\hat{b}_{w}}\right)\right)\left(i \omega+\frac{\gamma_{c}}{\gamma_{p}}\right)\left(i \omega+\frac{\beta\left(1+B_{x} \frac{\gamma_{c}}{\gamma_{p}}\right)}{\gamma_{p}\left(\alpha+\gamma_{c}\left(1+B_{x} \frac{\gamma_{c}}{\gamma_{p}}\right)\right)}\right) \\
& =-\frac{\beta}{\gamma_{p}^{2}} \frac{\alpha\left(1+B_{x} \frac{\gamma_{c}}{\gamma_{p}}\right)}{\left(\alpha+\gamma_{c}\left(1+B_{x} \frac{\gamma_{c}}{\gamma_{p}}\right)\right)}\left(1+i \omega \frac{\gamma_{p}}{\hat{b}_{w}}\right) .
\end{aligned}
$$

Taking the strong antithetic binding limit where $|i \omega| \ll \frac{\beta\left(1+B_{x} \frac{\gamma_{c}}{\gamma_{p}}\right)}{\gamma_{p}\left(\alpha+\gamma_{c}\left(1+B_{x} \frac{\gamma_{c}}{\gamma_{p}}\right)\right)}$, we have

$$
\left(1+B_{x} \frac{\gamma_{c}}{\gamma_{p}}-\frac{\gamma_{p}}{\hat{b}_{w}} \omega^{2}+i \omega\left(1+B_{x}+\frac{\gamma_{p}}{\hat{b}_{w}}\right)\right)(1+i \omega)\left(i \omega+\frac{\gamma_{c}}{\gamma_{p}}\right)=-\frac{\alpha}{\gamma_{p}}\left(1+i \omega \frac{\gamma_{p}}{\hat{b}_{w}}\right) .
$$


The magnitude constraint is

$$
\left(\left(\left(1+B_{x} \frac{\gamma_{c}}{\gamma_{p}}-\frac{\gamma_{p}}{\hat{b}_{w}} \omega^{2}\right)^{2}+\omega^{2}\left(1+B_{x}+\frac{\gamma_{p}}{\hat{b}_{w}}\right)^{2}\right)^{0.5}\left(1+\omega^{2}\right)^{0.5}\left(\omega^{2}+\frac{\gamma_{c}^{2}}{\gamma_{p}^{2}}\right)^{0.5}=\frac{\alpha}{\gamma_{p}}\left(1+\frac{\gamma_{p}^{2}}{\hat{b}_{w}^{2}} \omega^{2}\right)^{0.5}\right.
$$

and the phase constraint is

$$
\tan ^{-1}\left(\frac{\omega\left(1+B_{x}+\frac{\gamma_{p}}{\hat{b}_{w}}\right)}{1+B_{x} \frac{\gamma_{c}}{\gamma_{p}}-\frac{\gamma_{p}}{\hat{b}_{w}} \omega^{2}}\right)+\tan ^{-1}(\omega)+\tan ^{-1}\left(\frac{\gamma_{p}}{\gamma_{c}} \omega\right)=\tan ^{-1}\left(\frac{\gamma_{p}}{\hat{b}_{w}} \omega\right)+\pi+2 k \pi .
$$

for some integer $k$. Rearranging, we have

$$
\tan ^{-1}\left(\frac{\omega\left(1+B_{x}+\frac{\gamma_{p}}{\hat{b}_{w}}\right)}{1+B_{x} \frac{\gamma_{c}}{\gamma_{p}}-\frac{\gamma_{p}}{\hat{b}_{w}} \omega^{2}}\right)=\tan ^{-1}\left(\frac{\frac{\gamma_{p}}{\hat{b}_{w}} \omega\left(1-\frac{\gamma_{p}}{\gamma_{c}} \omega^{2}\right)-\left(1+\frac{\gamma_{p}}{\gamma_{c}}\right) \omega}{1-\frac{\gamma_{p}}{\gamma_{c}} \omega^{2}+\left(1+\frac{\gamma_{p}}{\gamma_{c}}\right) \frac{\gamma_{p}}{\hat{b}_{w}} \omega^{2}}\right)+\pi+2 k \pi
$$

Taking the tangent of both sides and ignoring the trivial solution, we have

$$
\frac{\left(1+B_{x}+\frac{\gamma_{p}}{\hat{b}_{w}}\right)}{1+B_{x} \frac{\gamma_{c}}{\gamma_{p}}-\frac{\gamma_{p}}{\hat{b}_{w}} \omega^{2}}=\frac{\frac{\gamma_{p}}{\hat{b}_{w}}\left(1-\frac{\gamma_{p}}{\gamma_{c}} \omega^{2}\right)-\left(1+\frac{\gamma_{p}}{\gamma_{c}}\right)}{1-\frac{\gamma_{p}}{\gamma_{c}} \omega^{2}+\left(1+\frac{\gamma_{p}}{\gamma_{c}}\right) \frac{\gamma_{p}}{\hat{b}_{w}} \omega^{2}}
$$

Multiplying out fractions, we have

$$
\left(1+B_{x}+\frac{\gamma_{p}}{\hat{b}_{w}}\right)\left(1-\frac{\gamma_{p}}{\gamma_{c}} \omega^{2}+\left(1+\frac{\gamma_{p}}{\gamma_{c}}\right) \frac{\gamma_{p}}{\hat{b}_{w}} \omega^{2}\right)=\left(\frac{\gamma_{p}}{\hat{b}_{w}}\left(1-\frac{\gamma_{p}}{\gamma_{c}} \omega^{2}\right)-\left(1+\frac{\gamma_{p}}{\gamma_{c}}\right)\right)\left(1+B_{x} \frac{\gamma_{c}}{\gamma_{p}}-\frac{\gamma_{p}}{\hat{b}_{w}} \omega^{2}\right) .
$$

Expanding, we have

$$
\begin{aligned}
& \left(1+B_{x}+\frac{\gamma_{p}}{\hat{b}_{w}}\right)+\left(1+B_{x}+\frac{\gamma_{p}}{\hat{b}_{w}}\right)\left(\frac{\gamma_{p}}{\hat{b}_{w}}+\frac{\gamma_{p}}{\gamma_{c}} \frac{\gamma_{p}}{\hat{b}_{w}}-\frac{\gamma_{p}}{\gamma_{c}}\right) \omega^{2} \\
& =-\left(1+\frac{\gamma_{p}}{\gamma_{c}}-\frac{\gamma_{p}}{\hat{b}_{w}}\right)\left(1+B_{x} \frac{\gamma_{c}}{\gamma_{p}}\right)+\left(1+\frac{\gamma_{p}}{\gamma_{c}}-\frac{\gamma_{p}}{\hat{b}_{w}}\right) \frac{\gamma_{p}}{\hat{b}_{w}} \omega^{2}-\left(1+B_{x} \frac{\gamma_{c}}{\gamma_{p}}\right) \frac{\gamma_{p}}{\hat{b}_{w}} \frac{\gamma_{p}}{\gamma_{c}} \omega^{2}+\frac{\gamma_{p}^{2}}{\hat{b}_{w}^{2}} \frac{\gamma_{p}}{\gamma_{c}} \omega^{4}
\end{aligned}
$$

Simplifying, we have

$$
-\frac{\gamma_{p}^{2}}{\hat{b}_{w}^{2}} \omega^{4}+\left[\left(1+B_{x}+\frac{\gamma_{p}}{\hat{b}_{w}}\right)\left(\frac{\gamma_{c}}{\hat{b}_{w}}+\frac{\gamma_{p}}{\hat{b}_{w}}-1\right)+\left(B_{x}-1+\frac{\gamma_{p}}{\hat{b}_{w}}\right) \frac{\gamma_{c}}{\hat{b}_{w}}\right] \omega^{2}+\left[2+2 B_{x}+\frac{\gamma_{p}}{\gamma_{c}}+B_{x} \frac{\gamma_{c}}{\gamma_{p}}-B_{x} \frac{\gamma_{c}}{b_{w}}\right] \frac{\gamma_{c}}{\gamma_{p}}=0
$$

Solving, we have

$$
\begin{aligned}
\omega^{2} & =\frac{\hat{b}_{w}^{2}}{2 \gamma_{p}^{2}}\left[A+\sqrt{A^{2}+4 \frac{\gamma_{p}^{2}}{\hat{b}_{w}^{2}} \frac{\gamma_{c}}{\gamma_{p}}\left[2+2 B_{x}+\frac{\gamma_{p}}{\gamma_{c}}+B_{x} \frac{\gamma_{c}}{\gamma_{p}}-B_{x} \frac{\gamma_{c}}{b_{w}}\right]}\right] \\
A & =\left[\left(1+B_{x}+\frac{\gamma_{p}}{\hat{b}_{w}}\right)\left(\frac{\gamma_{c}}{\hat{b}_{w}}+\frac{\gamma_{p}}{\hat{b}_{w}}-1\right)+\left(B_{x}-1+\frac{\gamma_{p}}{\hat{b}_{w}}\right) \frac{\gamma_{c}}{\hat{b}_{w}}\right]
\end{aligned}
$$

Using the same approach as in previous sections, the magnitude constraint leads to the stability constraint

$$
\gamma_{p}^{3}>\frac{\theta_{1} \theta_{2} k}{\left(1+\omega^{2}\right)^{0.5}\left(\omega^{2}+\frac{\gamma_{c}^{2}}{\gamma_{p}^{2}}\right)^{0.5}}\left(\frac{1+\frac{\gamma_{p}^{2}}{b_{w}^{2}} \omega^{2}}{\left(\left(1+B_{x} \frac{\gamma_{c}}{\gamma_{p}}-\frac{\gamma_{p}}{\hat{b}_{w}} \omega^{2}\right)^{2}+\omega^{2}\left(1+B_{x}+\frac{\gamma_{p}}{\hat{b}_{w}}\right)^{2}\right.}\right)^{0.5}
$$

using $\omega$ from (S28). This can be rewritten

$$
\frac{\alpha}{\gamma_{p}}<\left(1+\omega^{2}\right)^{0.5}\left(\omega^{2}+\frac{\gamma_{c}^{2}}{\gamma_{p}^{2}}\right)^{0.5}\left(\frac{\left(\left(1+B_{x} \frac{\gamma_{c}}{\gamma_{p}}-\frac{\gamma_{p}}{\hat{b}_{w}} \omega^{2}\right)^{2}+\omega^{2}\left(1+B_{x}+\frac{\gamma_{p}}{\hat{b}_{w}}\right)^{2}\right.}{1+\frac{\gamma_{p}^{2}}{b_{w}^{2}} \omega^{2}}\right)^{0.5}
$$


From SI3.1, we have there steady state error

$$
\frac{x_{2 n}-x_{2}}{x_{2 n}}=\frac{1}{1+\Omega_{x}}
$$

where

$$
\Omega_{x}=\frac{\alpha}{\gamma_{c}\left(1+B_{x} \frac{\gamma_{c}}{\gamma_{p}}\right)}
$$

and so the steady state error constraint is

$$
\Omega_{x}<\frac{\gamma_{p}}{\gamma_{c}} \frac{\left(1+\omega^{2}\right)^{0.5}}{\left(1+B_{x} \frac{\gamma_{c}}{\gamma_{p}}\right)}\left(\omega^{2}+\frac{\gamma_{c}^{2}}{\gamma_{p}^{2}}\right)^{0.5}\left(\frac{\left(\left(1+B_{x} \frac{\gamma_{c}}{\gamma_{p}}-\frac{\gamma_{p}}{\hat{b}_{w}} \omega^{2}\right)^{2}+\omega^{2}\left(1+B_{x}+\frac{\gamma_{p}}{\hat{b}_{w}}\right)^{2}\right.}{1+\frac{\gamma_{p}^{2}}{b_{w}^{2}} \omega^{2}}\right)^{0.5}
$$

where $\omega$ is given in (S28).

If we set $\hat{b}_{w}=\gamma_{p}$ for simplicity then $\omega^{2}=1+2\left(1+B_{x}\right) \frac{\gamma_{c}}{\gamma_{p}}$ and

$$
\Omega_{x}<\frac{\gamma_{p}}{\gamma_{c}} \frac{2+B_{x}}{1+B_{x} \frac{\gamma_{c}}{\gamma_{p}}}\left(1+\frac{\gamma_{c}^{2}}{\gamma_{p}^{2}}+2\left(1+B_{x}\right) \frac{\gamma_{c}}{\gamma_{p}}\right)
$$

This equation can be rewritten

$$
\Omega_{x}<\frac{\gamma_{p}}{\gamma_{c}} \frac{2+B_{x}}{1+B_{x} \frac{\gamma_{c}}{\gamma_{p}}}\left(\left(1+\frac{\gamma_{c}}{\gamma_{p}}\right)^{2}+2 B_{x} \frac{\gamma_{c}}{\gamma_{p}}\right)
$$

\section{S13.3. Rapid $x_{1}$ Buffering with Degradation can enable near-perfect adaptation}

In this section, we analyse the ability of buffering at $x_{1}$ to enable near perfect adaptation by stabilising antithetic integral feedback. This section uses identical methodology and obtains equivalent results to SI3.1.

Consider the model with $x_{1}$ buffering and dilution

$$
\begin{aligned}
\dot{x}_{1} & =\theta_{1} z_{1}-\gamma_{p} x_{1}-b_{i} x_{1}+b_{w} w_{x} \\
\dot{x}_{2} & =k x_{1}-\gamma_{p} x_{2} \\
\dot{w}_{x} & =b_{i} x_{1}-b_{w} w_{x}-\gamma_{i} w_{x} \\
\dot{z}_{1} & =\mu-\eta z_{1} z_{2} \\
\dot{z}_{2} & =\theta_{2} x_{2}-\eta z_{1} z_{2}
\end{aligned}
$$

where $w_{x}$ is the buffering species of $x_{1}$, and $b_{i}, b_{w}$ are the kinetic rates for the buffering reactions. Assuming rapid buffering and using the same methodology as previous sections, the reduced model is

$$
\begin{aligned}
\left(1+B_{i}\right) \dot{x}_{1} & =\theta_{1} z_{1}-\left(\gamma_{p}+B_{i} \gamma_{i}\right) x_{1} \\
\dot{x}_{2} & =k x_{1}-\gamma_{p} x_{2} \\
\dot{z}_{1} & =\mu-\eta z_{1} z_{2}-\gamma_{c} z_{1} \\
\dot{z}_{2} & =\theta_{2} x_{2}-\eta z_{1} z_{2}-\gamma_{c} z_{2} .
\end{aligned}
$$

where $B_{i}=\frac{b_{i}}{b_{w}+\gamma_{i}}$ is the buffer equilibrium ratio.

\section{S13.3.a. Steady State Analysis}

We next analyse the steady state of the system. We have

$$
\dot{z}_{1}-\dot{z}_{2}=\mu-\theta_{2} x_{2}-\gamma_{c} z_{1}+\gamma_{c} z_{2}
$$


and so the steady state of the output is

$$
x_{2}=\frac{\mu}{\theta_{2}}-\frac{\gamma_{c}}{\theta_{2}} z_{1}+\frac{\gamma_{c}}{\theta_{2}} z_{2}
$$

We also have the steady state

$$
z_{2}=\frac{1}{\eta}\left(\frac{\mu}{z_{1}}-\gamma_{c}\right)
$$

and so

$$
x_{2}=\frac{\mu}{\theta_{2}}-\frac{\gamma_{c}^{2}}{\theta_{2} \eta}-\frac{\gamma_{c}}{\theta_{2}} z_{1}+\frac{\gamma_{c} \mu}{\theta_{2} \eta} \frac{1}{z_{1}} .
$$

Now at steady state we have

$$
\begin{aligned}
& x_{1}=\frac{\gamma_{p}}{k} x_{2} \\
& z_{1}=\frac{\gamma_{p}}{\theta_{1}}\left(1+B_{i} \frac{\gamma_{i}}{\gamma_{p}}\right) x_{1}=\frac{\gamma_{p}^{2}}{\theta_{1} k}\left(1+B_{i} \frac{\gamma_{i}}{\gamma_{p}}\right) x_{2}=\frac{\theta_{2}}{\alpha}\left(1+B_{i} \frac{\gamma_{i}}{\gamma_{p}}\right) x_{2}
\end{aligned}
$$

where $\alpha=\frac{\theta_{1} \theta_{2} k}{\gamma_{p}^{2}}$. Substituting, we have

$$
\left(1+\frac{\gamma_{c}}{\alpha}\left(1+B_{i} \frac{\gamma_{i}}{\gamma_{p}}\right)\right) x_{2}^{2}=\left(\frac{\mu}{\theta_{2}}-\frac{\gamma_{c}^{2}}{\theta_{2} \eta}\right) x_{2}+\frac{\gamma_{c} \mu \alpha}{\theta_{2}^{2} \eta\left(1+B_{i} \frac{\gamma_{i}}{\gamma_{p}}\right)} .
$$

Assuming strong binding

$$
\eta \gg \frac{\gamma_{c}^{2}}{\mu}, \frac{\gamma_{c} \mu \alpha}{\theta_{2}^{2}\left(1+B_{i} \frac{\gamma_{i}}{\gamma_{p}}\right)}
$$

we have

$$
\left(1+\frac{\gamma_{c}}{\alpha}\left(1+B_{i} \frac{\gamma_{i}}{\gamma_{p}}\right)\right) x_{2}^{2}=\frac{\mu}{\theta_{2}} x_{2}
$$

and so, ignoring the solution $x_{1}=0$, the steady state is

$$
\begin{aligned}
& x_{2}=\frac{\mu}{\theta_{2}} \frac{1}{1+\frac{\gamma_{c}}{\alpha}\left(1+B_{i} \frac{\gamma_{i}}{\gamma_{p}}\right)} \\
& x_{1}=\frac{\gamma_{p}}{k} \frac{\mu}{\theta_{2}} \frac{1}{1+\frac{\gamma_{c}}{\alpha}\left(1+B_{i} \frac{\gamma_{i}}{\gamma_{p}}\right)}, \quad z_{1}=\frac{\mu\left(1+B_{i} \frac{\gamma_{i}}{\gamma_{p}}\right)}{\alpha+\gamma_{c}\left(1+B_{i} \frac{\gamma_{i}}{\gamma_{p}}\right)}, \quad z_{2}=\frac{\alpha}{\eta\left(1+B_{i} \frac{\gamma_{i}}{\gamma_{p}}\right)} .
\end{aligned}
$$

The steady state error of $x_{2}$ is

$$
\frac{x_{2 n}-x_{2}}{x_{2 n}}=\frac{1}{1+\frac{\alpha}{\gamma_{c}\left(1+B_{i} \frac{\gamma_{i}}{\gamma p}\right)}}
$$

\section{S13.3.b. Stability Analysis}

We next study the stability of the system. If we linearise about the steady state, we have

$$
\begin{aligned}
\left(1+B_{i}\right) \Delta \dot{x}_{1} & =\theta_{1} \Delta z_{1}-\gamma_{p}\left(1+B_{i} \frac{\gamma_{i}}{\gamma_{p}}\right) \Delta x_{1} \\
\Delta \dot{x}_{2} & =k \Delta x_{1}-\gamma_{p} \Delta x_{2} \\
\Delta \dot{z}_{1} & =-\eta \bar{z}_{2} \Delta z_{1}-\eta \bar{z}_{1} \Delta z_{2}-\gamma_{c} \Delta z_{1} \\
\Delta \dot{z}_{2} & =\theta_{2} \Delta x_{2}-\eta \bar{z}_{2} \Delta z_{1}-\eta \bar{z}_{1} \Delta z_{2}-\gamma_{c} \Delta z_{2} .
\end{aligned}
$$


This system can be rewritten as

$$
\begin{aligned}
\Delta \dot{x}_{1} & =\theta_{1} \Delta z_{1}-\gamma_{p} \Delta x_{1} \\
\left(1+B_{i}\right) \Delta \dot{x}_{2} & =k \Delta x_{1}-\gamma_{p}\left(1+B_{i} \frac{\gamma_{i}}{\gamma_{p}}\right) \Delta x_{2} \\
\Delta \dot{z}_{1} & =-\left(\frac{\alpha}{\left(1+B_{i} \frac{\gamma_{i}}{\gamma_{p}}\right)}+\gamma_{c}\right) \Delta z_{1}-\frac{\beta\left(1+B_{i} \frac{\gamma_{i}}{\gamma_{p}}\right)}{\alpha+\gamma_{c}\left(1+B_{i} \frac{\gamma_{i}}{\gamma_{p}}\right)} \Delta z_{2} \\
\Delta \dot{z}_{2} & =\theta_{2} \Delta x_{2}-\frac{\alpha}{\left(1+B_{i} \frac{\gamma_{i}}{\gamma_{p}}\right)} \Delta z_{1}-\left(\frac{\beta\left(1+B_{i} \frac{\gamma_{i}}{\gamma_{p}}\right)}{\alpha+\gamma_{c}\left(1+B_{i} \frac{\gamma_{i}}{\gamma_{p}}\right)}+\gamma_{c}\right) \Delta z_{2} .
\end{aligned}
$$

where $\alpha=\frac{\theta_{1} \theta_{2} k}{\gamma_{p}^{2}}$ and $\beta=\eta \mu$. Taking the Laplace transforms, we have

$$
\begin{aligned}
& \left(1+B_{i}\right)\left(s+\frac{\gamma_{p}+B_{i} \gamma_{i}}{1+B_{i}}\right) X_{1}=\theta_{1} Z_{1} \\
& \left(s+\gamma_{p}\right) X_{2}=k X_{1} \\
& \left(s+\frac{\alpha}{\left(1+B_{i} \frac{\gamma_{i}}{\gamma_{p}}\right)}+\gamma_{c}\right) Z_{1}=-\frac{\beta\left(1+B_{i} \frac{\gamma_{i}}{\gamma_{p}}\right)}{\alpha+\gamma_{c}\left(1+B_{i} \frac{\gamma_{i}}{\gamma_{p}}\right)} Z_{2} \\
& \left(s+\frac{\beta\left(1+B_{i} \frac{\gamma_{i}}{\gamma_{p}}\right)}{\alpha+\gamma_{c}\left(1+B_{i} \frac{\gamma_{i}}{\gamma_{p}}\right)}+\gamma_{c}\right) Z_{2}=\theta_{2} X_{2}-\frac{\alpha}{\left(1+B_{i} \frac{\gamma_{i}}{\gamma_{p}}\right)} Z_{1} .
\end{aligned}
$$

where $X_{1}, X_{2}, Z_{1}$ and $Z_{2}$ are the Laplace transforms for $\Delta x_{1}, \Delta x_{2}, \Delta z_{1}$ and $\Delta z_{2}$. Substituting, we have

$$
\begin{aligned}
& \left(1+B_{i}\right)\left(s+\gamma_{p}\right)\left(s+\frac{\gamma_{p}+B_{i} \gamma_{i}}{1+B_{i}}\right) X_{2}=\theta_{1} k Z_{1} \\
& \left(s+\frac{\alpha}{\left(1+B_{i} \frac{\gamma_{i}}{\gamma_{p}}\right)}+\gamma_{c}\right)\left(s+\frac{\beta\left(1+B_{i} \frac{\gamma_{i}}{\gamma_{p}}\right)}{\alpha+\gamma_{c}\left(1+B_{i} \frac{\gamma_{i}}{\gamma_{p}}\right)}+\gamma_{c}\right) Z_{1} \\
& =-\frac{\beta\left(1+B_{i} \frac{\gamma_{i}}{\gamma_{p}}\right)}{\alpha+\gamma_{c}\left(1+B_{i} \frac{\gamma_{i}}{\gamma_{p}}\right)}\left(\theta_{2} X_{2}-\frac{\alpha}{\left(1+B_{i} \frac{\gamma_{i}}{\gamma_{p}}\right)} Z_{1}\right)
\end{aligned}
$$

Rewriting and substituting, we have

$$
\begin{aligned}
& \left(1+B_{i}\right)\left(s+\gamma_{p}\right)\left(s+\frac{\gamma_{p}+B_{i} \gamma_{i}}{1+B_{i}}\right)\left[\left(s+\gamma_{c}\right)\left(s+\frac{\alpha}{\left(1+B_{i} \frac{\gamma_{i}}{\gamma_{p}}\right)}+\frac{\beta\left(1+B_{i} \frac{\gamma_{i}}{\gamma_{p}}\right)}{\alpha+\gamma_{c}\left(1+B_{i} \frac{\gamma_{i}}{\gamma_{p}}\right)}+\gamma_{c}\right)\right] X_{2} \\
& =-\frac{\beta\left(1+B_{i} \frac{\gamma_{i}}{\gamma_{p}}\right)}{\alpha+\gamma_{c}\left(1+B_{i} \frac{\gamma_{i}}{\gamma_{p}}\right)} \theta_{1} \theta_{2} k X_{2}
\end{aligned}
$$

The above equation is equivalent to that for $x_{2}$ buffering (see SI3.1), and so for strong integral binding we have the equivalent stability constraint and steady state error

$$
\begin{aligned}
\frac{x_{2 n}-x_{2}}{x_{2 n}} & =\frac{1}{1+\Omega_{i}} \\
\Omega_{i} & <\left(1+\frac{\gamma_{c}}{\gamma_{p}}\right)(1+A)\left(\frac{\gamma_{p}}{\gamma_{c}}+A^{-1}\right) \\
A & =\frac{1+\frac{\gamma_{i}}{\gamma_{p}} B_{i}}{1+B_{i}} .
\end{aligned}
$$




\section{S13.4. Rapid $z_{1}$ Buffering with Degradation has a trade-off due to leaky integration}

In this section, we analyse the trade-offs for rapid buffering at $z_{1}$ on stability and the steady state error from perfect adaptation. For buffering at $z_{1}$ with dilution, we use the model

$$
\begin{aligned}
& \dot{x}_{1}=\theta_{1} z_{1}-\gamma_{p} x_{1} \\
& \dot{x}_{2}=k x_{1}-\gamma_{p} x-\gamma_{p} x_{2} \\
& \dot{z}_{1}=\mu-\eta z_{1} z_{2}-\gamma_{c} z_{1}-b_{z} z_{1}+b_{w} w \\
& \dot{z}_{2}=\theta_{2} x_{2}-\eta z_{1} z_{2}-\gamma_{c} z_{2} \\
& \dot{w}=b_{z} z_{1}-b_{w} w-\gamma_{c} w
\end{aligned}
$$

where $x_{2}$ is the output concentration being controlled, $x_{1}$ is another concentration in the process being controlled, and $z_{1}$ and $z_{2}$ represent the molecular species involved in the perfect adaptation mechanism. Assuming that the buffer is rapid then $w$ is at quasi-steady state then

$$
w=B_{1} z_{1} \quad B_{1}=\frac{b_{z}}{b_{w}+\gamma_{c}}
$$

If $x_{T}=w+z_{1}$ is the slow variable then $x_{T}=\left(1+B_{1}\right) z_{1}$. Thus $\dot{x}_{T}=\left(1+B_{1}\right) \dot{z}_{1}$ and so

$$
\left(1+B_{1}\right) \dot{z}_{1}=\mu-\eta z_{1} z_{2}-\gamma_{c}\left(1+B_{1}\right) z_{1} .
$$

Thus we have

$$
\begin{aligned}
\dot{x}_{1} & =\theta_{1} z_{1}-\gamma_{p} x_{1} \\
\dot{x}_{2} & =k x_{1}-\gamma_{p} x_{2} \\
\left(1+B_{1}\right) \dot{z}_{1} & =\mu-\eta z_{1} z_{2}-\gamma_{c}\left(1+B_{1}\right) z_{1} \\
\dot{z}_{2} & =\theta_{2} x_{2}-\eta z_{1} z_{2}-\gamma_{c} z_{2} .
\end{aligned}
$$

\section{S13.4.a. Steady State Analysis}

We next determine the steady state and and any error from perfect adaptation. For the case of dilution, we have

$$
\left(1+B_{1}\right) \dot{z}_{1}-\dot{z}_{2}=\mu-\theta_{2} x_{2}-\gamma_{c}\left(\left(1+B_{1}\right) z_{1}-z_{2}\right)=0
$$

resulting in

$$
x_{2}=\frac{\mu}{\theta_{2}}-\frac{\gamma_{c}}{\theta_{2}}\left(1+B_{1}\right) z_{1}+\frac{\gamma_{c}}{\theta_{2}} z_{2}
$$

We also have

$$
z_{2}=\frac{1}{\eta}\left(\frac{\mu}{z_{1}}-\gamma_{c}\left(1+B_{1}\right)\right)
$$

and so

$$
x_{2}=\frac{\mu}{\theta_{2}}-\frac{\gamma_{c}^{2}}{\theta_{2} \eta}\left(1+B_{1}\right)-\frac{\gamma_{c}}{\theta_{2}}\left(1+B_{1}\right) z_{1}+\frac{\gamma_{c} \mu}{\theta_{2} \eta} \frac{1}{z_{1}}
$$

Now at steady state we have

$$
\begin{aligned}
& x_{1}=\frac{\gamma_{p}}{k} x_{2} \\
& z_{1}=\frac{\gamma_{p}}{\theta_{1}} x_{1}=\frac{\gamma_{p}^{2}}{\theta_{1} k} x_{2}=\frac{\theta_{2}}{\alpha} x_{2} .
\end{aligned}
$$


where $\alpha=\frac{\theta_{1} \theta_{2} k}{\gamma_{p}^{2}}$. Substituting, we have

$$
\left(1+\frac{\gamma_{c}}{\alpha}\left(1+B_{1}\right)\right) x_{2}^{2}=\left(\frac{\mu}{\theta_{2}}-\frac{\gamma_{c}^{2}}{\theta_{2} \eta}\left(1+B_{1}\right)\right) x_{2}+\frac{\gamma_{c} \mu \alpha}{\theta_{2}^{2} \eta}
$$

Assuming strong binding of the sequestration mechanism

$$
\eta \gg \frac{\gamma_{c}^{2}\left(1+B_{1}\right)}{\mu}, \frac{\gamma_{c} \mu \alpha}{\theta_{2}^{2}}
$$

we have

$$
\left(1+\frac{\gamma_{c}}{\alpha}\left(1+B_{1}\right)\right) x_{2}^{2}=\frac{\mu}{\theta_{2}} x_{2}
$$

and so, ignoring the zero solution, the steady state is

$$
\begin{gathered}
x_{2}=\frac{\mu}{\theta_{2}} \frac{1}{1+\frac{\gamma_{c}}{\alpha}\left(1+B_{1}\right)} \\
x_{1}=\frac{\gamma_{p}}{k} \frac{\mu}{\theta_{2}} \frac{1}{1+\frac{\gamma_{c}}{\alpha}\left(1+B_{1}\right)}, \quad z_{1}=\frac{\mu}{\alpha+\gamma_{c}\left(1+B_{1}\right)}, \quad z_{2}=\frac{\alpha}{\eta} .
\end{gathered}
$$

The steady state error of $x_{2}$ is

$$
\frac{x_{2 n}-x_{2}}{x_{2 n}}=\frac{1}{1+\Omega_{1}}, \quad \Omega_{1}=\frac{\alpha}{\gamma_{c}\left(1+B_{1}\right)} \text {. }
$$

We can see that increasing $B_{1}$ increases the steady state error of $x_{2}$ when there is degradation/dilution of $z_{1}$ and $z_{2}$.

\section{SI3.4.b. Stability Analysis}

We next study the stability of the system with degradation. If we linearise about the steady states, we have

$$
\begin{aligned}
\Delta \dot{x}_{1} & =\theta_{1} \Delta z_{1}-\gamma_{p} \Delta x_{1} \\
\Delta \dot{x}_{2} & =k \Delta x_{1}-\gamma_{p} \Delta x_{2} \\
\left(1+B_{1}\right) \Delta \dot{z}_{1} & =-\eta \bar{z}_{2} \Delta z_{1}-\eta \bar{z}_{1} \Delta z_{2}-\gamma_{c}\left(1+B_{1}\right) \Delta z_{1} \\
\Delta \dot{z}_{2} & =\theta_{2} \Delta x_{2}-\eta \bar{z}_{2} \Delta z_{1}-\eta \bar{z}_{1} \Delta z_{2}-\gamma_{c} \Delta z_{2} .
\end{aligned}
$$

This system can be rewritten as

$$
\begin{aligned}
\Delta \dot{x}_{1} & =\theta_{1} \Delta z_{1}-\gamma_{p} \Delta x_{1} \\
\Delta \dot{x}_{2} & =k \Delta x_{1}-\gamma_{p} \Delta x_{2} \\
\left(1+B_{1}\right) \Delta \dot{z}_{1} & =-\left(\alpha+\gamma_{c}\left(1+B_{1}\right)\right) \Delta z_{1}-\frac{\beta}{\alpha+\gamma_{c}\left(1+B_{1}\right)} \Delta z_{2} \\
\Delta \dot{z}_{2} & =\theta_{2} \Delta x_{2}-\alpha \Delta z_{1}-\left(\frac{\beta}{\alpha+\gamma_{c}\left(1+B_{1}\right)}+\gamma_{c}\right) \Delta z_{2}
\end{aligned}
$$

where

$$
\alpha=\frac{\theta_{1} \theta_{2} k}{\gamma_{p}^{2}}, \quad \beta=\eta \mu
$$


Taking the Laplace transforms, we have

$$
\begin{aligned}
& \left(s+\gamma_{p}\right) X_{1}=\theta_{1} Z_{1} \\
& \left(s+\gamma_{p}\right) X_{2}=k X_{1} \\
& \left(\left(1+B_{1}\right) s+\alpha+\gamma_{c}\left(1+B_{1}\right)\right) Z_{1}=-\frac{\beta}{\alpha+\gamma_{c}\left(1+B_{1}\right)} Z_{2} \\
& \left(s+\frac{\beta}{\alpha+\gamma_{c}\left(1+B_{1}\right)}+\gamma_{c}\right) Z_{2}=\theta_{2} X_{2}-\alpha Z_{1} .
\end{aligned}
$$

where $X_{1}, X_{2}, Z_{1}$ and $Z_{2}$ are the laplace transforms for $\Delta x_{1}, \Delta x_{2}, \Delta z_{1}$ and $\Delta z_{2}$. Substituting, we have

$$
\begin{aligned}
& \left(s+\gamma_{p}\right)^{2} X_{2}=\theta_{1} k Z_{1} \\
& \left(\left(1+B_{1}\right) s+\alpha+\gamma_{c}\left(1+B_{1}\right)\right)\left(s+\frac{\beta}{\alpha+\gamma_{c}\left(1+B_{1}\right)}+\gamma_{c}\right) Z_{1} \\
& =-\frac{\beta}{\alpha+\gamma_{c}\left(1+B_{1}\right)}\left(\theta_{2} X_{2}-\alpha Z_{1}\right) .
\end{aligned}
$$

Rewriting and substituting, we have

$$
\begin{aligned}
& \left(s+\gamma_{p}\right)^{2}\left[\left(s+\gamma_{c}\right)\left(\left(1+B_{1}\right) s+\alpha+\frac{\beta\left(1+B_{1}\right)}{\alpha+\gamma_{c}\left(1+B_{1}\right)}+\gamma_{c}\left(1+B_{1}\right)\right)\right] X_{2} \\
& =-\frac{\beta}{\alpha+\gamma_{c}\left(1+B_{1}\right)} \theta_{1} \theta_{2} k X_{2} .
\end{aligned}
$$

Taking the limit of strong binding $\left(\beta \gg \max \left\{\frac{\left(\alpha+\gamma_{c}\left(1+B_{1}\right)\right)^{2}}{1+B_{1}}, \frac{\gamma_{p}\left(\alpha+\gamma_{c}\left(1+B_{1}\right)\right)}{1+B_{1}}\right\}\right)$ then

$$
\left(1+B_{1}\right)\left(s+\gamma_{p}\right)^{2}\left(s+\gamma_{c}\right)\left(s+\frac{\beta}{\alpha+\gamma_{c}\left(1+B_{1}\right)}\right) X_{2}=-\frac{\beta}{\alpha+\gamma_{c}\left(1+B_{1}\right)} \theta_{1} \theta_{2} k X_{2} .
$$

Thus we have the characteristic equation

$$
\left(s+\gamma_{p}\right)^{2}\left(s+\gamma_{c}\right)\left(s+\frac{\beta}{\alpha+\gamma_{c}\left(1+B_{1}\right)}\right)+\frac{\beta \gamma_{p}^{2}}{\left(1+B_{1}\right)} \frac{\alpha}{\alpha+\gamma_{c}\left(1+B_{1}\right)}=0 .
$$

Substituting $s=\gamma_{p} \sigma$, we have

$$
(1+\sigma)^{2}\left(\sigma+\frac{\gamma_{c}}{\gamma_{p}}\right)\left(\sigma+\frac{\beta}{\gamma_{p}\left(\alpha+\gamma_{c}\left(1+B_{1}\right)\right)}\right)=-\frac{\beta}{\gamma_{p}^{2}\left(1+B_{1}\right)} \frac{\alpha}{\alpha+\gamma_{c}\left(1+B_{1}\right)} .
$$

Using the same argument as above, for the stability boundary with strong binding there is a negative real and complex pair of roots in the region $|\sigma| \ll \frac{\beta}{\gamma_{p}\left(\alpha+\gamma_{c}\left(1+B_{1}\right)\right)}$, as well as one large negative root.

To determine the boundary of stability, we next determine the conditions for which the roots are purely imaginary. Substituting $s=i \omega \gamma_{p}$, we have

$$
(1+i \omega)^{2}\left(i \omega+\frac{\gamma_{c}}{\gamma_{p}}\right)\left(i \omega+\frac{\beta}{\gamma_{p}\left(\alpha+\gamma_{c}\left(1+B_{1}\right)\right)}\right)=-\frac{\beta}{\gamma_{p}^{2}\left(1+B_{1}\right)} \frac{\alpha}{\alpha+\gamma_{c}\left(1+B_{1}\right)}
$$

Taking the strong antithetic binding limit where $|i \omega| \ll \frac{\beta}{\gamma_{p}\left(\alpha+\gamma_{c}\left(1+B_{1}\right)\right)}$, we have

$$
(1+i \omega)^{2}\left(i \omega+\frac{\gamma_{c}}{\gamma_{p}}\right)=-\frac{\alpha}{\gamma_{p}\left(1+B_{1}\right)} .
$$

The phase and magnitude constraints are

$$
\begin{aligned}
& \left(1+\omega^{2}\right)\left(\omega^{2}+\frac{\gamma_{c}^{2}}{\gamma_{p}^{2}}\right)^{0.5}=\frac{\alpha}{\gamma_{p}\left(1+B_{1}\right)} \\
& 2 \tan ^{-1}(\omega)+\tan ^{-1}\left(\frac{\gamma_{p}}{\gamma_{c}} \omega\right)=\pi+2 k \pi
\end{aligned}
$$


for some integer $k$. Solving the phase constraint, we have

$$
\tan ^{-1}\left(\frac{2 \omega+\frac{\gamma_{p}}{\gamma_{c}} \omega\left(1-\omega^{2}\right)}{1-\left(1+2 \frac{\gamma_{p}}{\gamma_{c}}\right) \omega^{2}}\right)=\pi+2 k \pi .
$$

For this, we require

$$
2 \omega+\frac{\gamma_{p}}{\gamma_{c}} \omega\left(1-\omega^{2}\right)
$$

which reduces to

$$
\omega=\sqrt{2 \frac{\gamma_{c}}{\gamma_{p}}+1}
$$

Substituting into the magnitude equation, we have

$$
\frac{\alpha}{\gamma_{p}\left(1+B_{1}\right)}=2\left(1+\frac{\gamma_{c}}{\gamma_{p}}\right)^{2}
$$

As a consequence, the stability constraint is

$$
\Omega_{1}=\frac{\alpha}{\gamma_{c}\left(1+B_{1}\right)}<2 \frac{\gamma_{p}}{\gamma_{c}}\left(1+\frac{\gamma_{c}}{\gamma_{p}}\right)^{2} .
$$

We can observe that increasing $B_{1}$ improves the stability constraint. However, the steady state error of $x_{2}$ is

$$
\begin{array}{r}
\frac{x_{2 n}-x_{2}}{x_{2 n}}=\frac{1}{1+\Omega_{1}} \\
\Omega_{1}=\frac{\alpha}{\gamma_{c}\left(1+B_{1}\right)} \\
\Omega_{1}<2 \frac{\gamma_{p}}{\gamma_{c}}\left(1+\frac{\gamma_{c}}{\gamma_{p}}\right)^{2}
\end{array}
$$

Thus there is a steady state error constraint that is independent of $B_{1}$, and so increasing $B_{1}$ does not enable the removal of leaky integration.

\section{SI3.5. Non-Rapid $z_{1}$ Buffering can allow Near Perfect Adaptation}

In this section, we analyse the ability of non-rapid buffering at $z_{1}$ to enable near perfect adaptation by stabilising antithetic integral feedback. We use the model

$$
\begin{aligned}
& \dot{x}_{1}=\theta_{1} z_{1}-\gamma_{p} x_{1} \\
& \dot{x}_{2}=k x_{1}-\gamma_{p} x_{2} \\
& \dot{z}_{1}=\mu-\eta z_{1} z_{2}-\gamma_{c} z_{1}-b_{z} z_{1}+b_{w} w \\
& \dot{z}_{2}=\theta_{2} x_{2}-\eta z_{1} z_{2}-\gamma_{c} z_{2} \\
& \dot{w}=b_{z} z_{1}-b_{w} w-\gamma_{c} w .
\end{aligned}
$$

where the buffer $w$ is not assumed to rapidly reach equilibrium. The steady state for (S33) is identical to the rapid case in SI3.4. The linearisation is

$$
\begin{aligned}
& \Delta \dot{x}_{1}=\theta_{1} \Delta z_{1}-\gamma_{p} \Delta x_{1} \\
& \Delta \dot{x}_{2}=k \Delta x_{1}-\gamma_{p} \Delta x_{2} \\
& \Delta \dot{z}_{1}=-\eta \bar{z}_{2} \Delta z_{1}-\eta \bar{z}_{1} \Delta z_{2}-\gamma_{c} \Delta z_{1}-b_{z} \Delta z_{1}+b_{w} \Delta w \\
& \Delta \dot{z}_{2}=\theta_{2} \Delta x_{2}-\eta \bar{z}_{2} \Delta z_{1}-\eta \bar{z}_{1} \Delta z_{2}-\gamma_{c} \Delta z_{2} \\
& \Delta \dot{w}=b_{z} \Delta z_{1}-\left(b_{w}+\gamma_{c}\right) \Delta w .
\end{aligned}
$$


This system can be rewritten

$$
\begin{aligned}
& \Delta \dot{x}_{1}=\theta_{1} \Delta z_{1}-\gamma_{p} \Delta x_{1} \\
& \Delta \dot{x}_{2}=k \Delta x_{1}-\gamma_{p} \Delta x_{2} \\
& \Delta \dot{z}_{1}=-\left(\alpha+\gamma_{c}\right) \Delta z_{1}-\frac{\beta}{\alpha+\gamma_{c}\left(1+B_{1}\right)} \Delta z_{2}-b_{z} \Delta z_{1}+b_{w} \Delta w \\
& \Delta \dot{z}_{2}=\theta_{2} \Delta x_{2}-\alpha \Delta z_{1}-\left(\frac{\beta}{\alpha+\gamma_{c}\left(1+B_{1}\right)}+\gamma_{c}\right) \Delta z_{2} \\
& \Delta \dot{w}=b_{z} \Delta z_{1}-\left(b_{w}+\gamma_{c}\right) \Delta w .
\end{aligned}
$$

where

$$
\alpha=\frac{\theta_{1} \theta_{2} k}{\gamma_{p}^{2}}, \quad \beta=\eta \mu .
$$

Taking the Laplace transform of $\Delta \dot{w}=b_{z} \Delta z_{1}-\left(b_{w}+\gamma_{c}\right) \Delta w$, we have

$$
W=\frac{b_{z}}{s+b_{w}+\gamma_{c}} Z_{1}
$$

where $W$ and $Z_{1}$ are the Laplace transforms of $w$ and $z_{1}$. We have

$$
\begin{aligned}
-b_{z} Z_{1}+b_{w} W & =-b_{z} Z_{1}+b_{w} \frac{b_{z}}{s+b_{w}+\gamma_{c}} Z_{1} \\
& =-B_{1} \frac{s+\gamma_{c}}{1+\frac{s}{b_{w}+\gamma_{c}}} Z_{1} \\
& =-C_{b}(s) Z_{1}
\end{aligned}
$$

where

$$
C_{b}=B_{1} \frac{s+\gamma_{c}}{1+\frac{s}{\hat{b}_{w}}}, \quad \hat{b}_{w}=b_{w}+\gamma_{c}
$$

Thus

$$
\begin{aligned}
& \left(s+\gamma_{p}\right) X_{1}=\theta_{1} Z_{1} \\
& \left(s+\gamma_{p}\right) X_{2}=k X_{1} \\
& \left(s+\alpha+\gamma_{c}+C_{b}\right) Z_{1}=-\frac{\beta}{\alpha+\gamma_{c}\left(1+B_{1}\right)} Z_{2} \\
& \left(s+\frac{\beta}{\alpha+\gamma_{c}\left(1+B_{1}\right)}+\gamma_{c}\right) Z_{2}=\theta_{2} X_{2}-\alpha Z_{1} .
\end{aligned}
$$

Combining, we have

$$
\begin{aligned}
& \left(s+\gamma_{p}\right)^{2} X_{2}=\theta_{1} k Z_{1} \\
& \left(s+\alpha+\gamma_{c}+C_{b}\right)\left(s+\frac{\beta}{\alpha+\gamma_{c}\left(1+B_{1}\right)}+\gamma_{c}\right) Z_{1}=-\frac{\beta}{\alpha+\gamma_{c}\left(1+B_{1}\right)}\left(\theta_{2} X_{2}-\alpha Z_{1}\right) .
\end{aligned}
$$

Simplifying, we have

$$
\begin{aligned}
& {\left[\left(s+\gamma_{c}\right)\left(s+\alpha+\frac{\beta}{\alpha+\gamma_{c}\left(1+B_{1}\right)}+\gamma_{c}\right)+C_{b}\left(s+\frac{\beta}{\alpha+\gamma_{c}\left(1+B_{1}\right)}+\gamma_{c}\right)\right] Z_{1}} \\
& =-\frac{\beta}{\alpha+\gamma_{c}\left(1+B_{1}\right)} \theta_{2} X_{2} .
\end{aligned}
$$

Taking the strong antithetic binding limit of the sequestration mechanism $\left(\beta \gg \max \left\{\left(\alpha+\gamma_{c}\right)\left(\alpha+\gamma_{c}\left(1+B_{1}\right)\right), \gamma_{p}\left(\alpha+\gamma_{c}\left(1+B_{1}\right)\right)\right\}\right)$, we have

$$
\left(s+\gamma_{c}+C_{b}\right)\left(s+\frac{\beta}{\alpha+\gamma_{c}\left(1+B_{1}\right)}\right) Z_{1}=-\frac{\beta}{\alpha+\gamma_{c}\left(1+B_{1}\right)} \theta_{2} X_{2}
$$


and so

$$
\left(s+\gamma_{p}\right)^{2}\left[\left(s+\gamma_{c}+C_{b}\right)\left(s+\frac{\beta}{\alpha+\gamma_{c}\left(1+B_{1}\right)}\right)\right] X_{2}=-\beta \gamma_{p}^{2} \frac{\alpha}{\alpha+\gamma_{c}\left(1+B_{1}\right)} X_{2}
$$

Rewriting $C_{b}$, we have

$$
\left[\left(s+\gamma_{p}\right)^{2}\left(s+\gamma_{c}\right) \frac{1+B_{1}+\frac{s}{\hat{b}_{w v}}}{1+\frac{s}{\hat{b}_{w}}}\left(s+\frac{\beta}{\alpha+\gamma_{c}\left(1+B_{1}\right)}\right)+\beta \gamma_{p}^{2} \frac{\alpha}{\alpha+\gamma_{c}\left(1+B_{1}\right)}\right] X_{2}=0
$$

or

$$
\left[\left(s+\gamma_{p}\right)^{2}\left(s+\gamma_{c}\right)\left(1+B_{1}+\frac{s}{\hat{b}_{w}}\right)\left(s+\frac{\beta}{\alpha+\gamma_{c}\left(1+B_{1}\right)}\right)+\beta \gamma_{p}^{2} \frac{\alpha\left(1+\frac{s}{\hat{b}_{w}}\right)}{\alpha+\gamma_{c}\left(1+B_{1}\right)}\right] X_{2}=0 .
$$

Substituting $s=i \omega \gamma_{p}$, we have

$$
\left(1+B_{1}+i \omega \frac{\gamma_{p}}{\hat{b}_{w}}\right)(1+i \omega)^{2}\left(\frac{\gamma_{c}}{\gamma_{p}}+i \omega\right)\left(i \omega+\frac{\beta}{\gamma_{p}\left(\alpha+\gamma_{c}\left(1+B_{1}\right)\right)}\right)+\frac{\beta}{\gamma_{p}^{2}} \frac{\alpha\left(1+i \omega \frac{\gamma_{p}}{\hat{b}_{w}}\right)}{\alpha+\gamma_{c}\left(1+B_{1}\right)}=0 .
$$

Taking the strong antithetic binding limit where $|i \omega| \ll \frac{\beta}{\gamma_{p}\left(\alpha+\gamma_{c}\left(1+B_{1}\right)\right)}$, we have

$$
\left(1+B_{1}+i \omega \frac{\gamma_{p}}{\hat{b}_{w}}\right)(1+i \omega)^{2}\left(\frac{\gamma_{c}}{\gamma_{p}}+i \omega\right)=-\frac{\alpha}{\gamma_{p}}\left(1+i \omega \frac{\gamma_{p}}{\hat{b}_{w}}\right) .
$$

The magnitude constraint is

$$
\left(1+B_{1}\right)\left(1+\frac{\gamma_{p}^{2}}{\hat{b}_{w}^{2}} \frac{\omega^{2}}{\left(1+B_{1}\right)^{2}}\right)^{0.5}\left(1+\omega^{2}\right)\left(\frac{\gamma_{c}^{2}}{\gamma_{p}^{2}}+\omega^{2}\right)^{0.5}=\frac{\alpha}{\gamma_{p}}\left(1+\omega^{2} \frac{\gamma_{p}^{2}}{\hat{b}_{w}^{2}}\right)^{0.5}
$$

and the phase constraint is

$$
2 \tan ^{-1}(\omega)+\tan ^{-1}\left(\frac{\gamma_{p}}{\gamma_{c}} \omega\right)+\tan ^{-1}\left(\frac{\gamma_{p}}{\hat{b}_{w}} \frac{1}{1+B_{1}} \omega\right)=\pi+\tan ^{-1}\left(\frac{\gamma_{p}}{\hat{b}_{w}} \omega\right)+2 k \pi .
$$

for some integer $k$. Using trigonometric identities, we have

$$
\tan ^{-1}\left(\frac{2 \omega+\frac{\gamma_{p}}{\gamma_{c}} \omega\left(1-\omega^{2}\right)}{1-\left(1+2 \frac{\gamma_{p}}{\gamma_{c}}\right) \omega^{2}}\right)=\pi+2 k \pi+\tan ^{-1}\left(\frac{\frac{B_{1}}{1+B_{1}} \frac{\gamma_{p}}{b_{w}} \omega}{1+\frac{1}{1+B_{1}} \frac{\gamma_{p}^{2}}{\hat{b}_{w}^{2}} \omega^{2}}\right) .
$$

This can be simplified to

$$
\frac{2 \omega+\frac{\gamma_{p}}{\gamma_{c}} \omega\left(1-\omega^{2}\right)}{1-\left(1+2 \frac{\gamma_{p}}{\gamma_{c}}\right) \omega^{2}}=\frac{\frac{B_{1}}{1+B_{1}} \frac{\gamma_{p}}{\hat{b}_{w}} \omega}{1+\frac{1}{1+B_{1}} \frac{\gamma_{p}^{2}}{\hat{b}_{w}^{2}} \omega^{2}}
$$

Ignoring the trivial solution $\omega=0$, we have

$$
\left(2+\frac{\gamma_{p}}{\gamma_{c}}-\frac{\gamma_{p}}{\gamma_{c}} \omega^{2}\right)\left(1+\frac{1}{1+B_{1}} \frac{\gamma_{p}^{2}}{\hat{b}_{w}^{2}} \omega^{2}\right)=\frac{B_{1}}{1+B_{1}} \frac{\gamma_{p}}{\hat{b}_{w}}\left(1-\left(1+2 \frac{\gamma_{p}}{\gamma_{c}}\right) \omega^{2}\right)
$$

Rewriting, we have

$$
-\frac{1}{1+B_{1}} \frac{\gamma_{p}^{2}}{\hat{b}_{w}^{2}} \frac{\gamma_{p}}{\gamma_{c}} \omega^{4}+\left[-\frac{\gamma_{p}}{\gamma_{c}}+\left(2+\frac{\gamma_{p}}{\gamma_{c}}\right) \frac{1}{1+B_{1}} \frac{\gamma_{p}^{2}}{\hat{b}_{w}^{2}}+\frac{B_{1}}{1+B_{1}} \frac{\gamma_{p}}{\hat{b}_{w}}\left(1+2 \frac{\gamma_{p}}{\gamma_{c}}\right)\right] \omega^{2}+\left(2+\frac{\gamma_{p}}{\gamma_{c}}\right)-\frac{B_{1}}{1+B_{1}} \frac{\gamma_{p}}{\hat{b}_{w}}=0 .
$$


Simplifying, we have

$$
-\frac{\gamma_{p}^{2}}{\hat{b}_{w}^{2}} \omega^{4}+\left[-\left(1+B_{1}\right)+\left(2 \frac{\gamma_{c}}{\gamma_{p}}+1\right) \frac{\gamma_{p}^{2}}{\hat{b}_{w}^{2}}+B_{1} \frac{\gamma_{p}}{\hat{b}_{w}}\left(\frac{\gamma_{c}}{\gamma_{p}}+2\right)\right] \omega^{2}+\left(2 \frac{\gamma_{c}}{\gamma_{p}}+1\right)\left(1+B_{1}\right)-B_{1} \frac{\gamma_{p}}{\hat{b}_{w}} \frac{\gamma_{c}}{\gamma_{p}}=0 .
$$

Solving, we have

$$
\begin{aligned}
\omega^{2} & =\frac{\hat{b}_{w}^{2}}{2 \gamma_{p}^{2}}\left[A+\sqrt{A^{2}+4 \frac{\gamma_{p}^{2}}{\hat{b}_{w}^{2}}\left(\left(2 \frac{\gamma_{c}}{\gamma_{p}}+1\right)\left(1+B_{1}\right)-B_{1} \frac{\gamma_{c}}{\hat{b}_{w}}\right)}\right] \\
A & =-\left(1+B_{1}\right)+\left(2 \frac{\gamma_{c}}{\gamma_{p}}+1\right) \frac{\gamma_{p}^{2}}{\hat{b}_{w}^{2}}+B_{1} \frac{\gamma_{p}}{\hat{b}_{w}}\left(\frac{\gamma_{c}}{\gamma_{p}}+2\right) .
\end{aligned}
$$

From the magnitude constraint S34, we have the stability constraint

$$
\left(1+B_{1}\right)\left(\frac{1+\frac{\gamma_{p}^{2}}{\hat{b}_{w}^{2}} \frac{\omega^{2}}{\left(1+B_{1}\right)^{2}}}{1+\omega^{2} \frac{\gamma_{p}^{2}}{\hat{b}_{w}^{2}}}\right)^{0.5}\left(1+\omega^{2}\right)\left(\frac{\gamma_{c}^{2}}{\gamma_{p}^{2}}+\omega^{2}\right)^{0.5}>\frac{\alpha}{\gamma_{p}}
$$

which can be rewritten in terms of steady state error $\Omega_{1}=\frac{\alpha}{\gamma_{c}\left(1+B_{1}\right)}$ as

$$
\Omega_{1}<\frac{\gamma_{p}}{\gamma_{c}}\left(1+\omega^{2}\right)\left(\frac{\gamma_{c}^{2}}{\gamma_{p}^{2}}+\omega^{2}\right)^{0.5}\left(\frac{1+\frac{\gamma_{p}^{2}}{\hat{b}_{w}^{2}} \frac{\omega^{2}}{\left(1+B_{1}\right)^{2}}}{1+\omega^{2} \frac{\gamma_{p}^{2}}{\hat{b}_{w}^{2}}}\right)^{0.5}
$$

For $\hat{b}_{w}=\gamma_{p}$, we have

$$
\begin{gathered}
A=\left(1+\frac{\gamma_{c}}{\gamma_{p}}\right) B_{1}+2 \frac{\gamma_{c}}{\gamma_{p}} \\
\omega^{2}=\left(1+\frac{\gamma_{c}}{\gamma_{p}}\right) B_{1}+1+2 \frac{\gamma_{c}}{\gamma_{p}}
\end{gathered}
$$

and the stability constraint

$$
\Omega_{1}<\frac{\gamma_{p}}{\gamma_{c}} \frac{\left(2+B_{1}\right)}{\left(1+B_{1}\right)}\left(1+B_{1}+\frac{\gamma_{c}}{\gamma_{p}}\right)\left(1+\frac{\gamma_{c}}{\gamma_{p}}\right)
$$

\section{SI4. CHARACTERISATION OF ANTITHETIC FEEDBACK WITH BUFFERING}

In this section we characterise the feedback in the system with antithetic feedback and buffering of the control species $z_{1}, z_{2}$.

\section{S14.1. Characterisation with Rapid Buffering}

We first characterise antithetic feedback with rapid buffering. We use the model

$$
\begin{aligned}
\dot{x}_{1} & =\theta_{1} z_{1}-\gamma_{p} x_{1} \\
\left(1+B_{x}\right) \dot{x}_{2} & =k x_{1}-\left(\gamma_{p}+B_{x} \gamma_{x}\right) x_{2} \\
\left(1+B_{1}\right) \dot{z}_{1} & =\mu-\eta z_{1} z_{2} \\
\left(1+B_{2}\right) \dot{z}_{2} & =\theta_{2} x_{2}-\eta z_{1} z_{2} .
\end{aligned}
$$


It can be observed that rapid buffers of the controller change the time-scale for $z_{1}$ and $z_{2}$. In integral feedback, the time-scale is inversely proportional to gain, and this relationship can be observed in the following characterisation of integral gain. We have

$$
\left(1+B_{1}\right) \dot{z}_{1}(t)-\left(1+B_{2}\right) \dot{z}_{2}(t)=\mu-\theta_{2} x_{2}
$$

Integrating, we have

$$
\left(1+B_{1}\right) z_{1}(t)-\left(1+B_{2}\right) z_{2}(t)=\theta_{2} \int_{0}^{t}\left(\frac{\mu}{\theta_{2}}-x_{2}\left(t^{\prime}\right)\right) d t^{\prime}
$$

Rearranging, we can observe that the feedback control input $u$ into the plant (i.e. $\left(x_{1}, x_{2}\right)$ subsystem) is

$$
u=\theta_{1} z_{1}=\underbrace{\frac{\theta_{1} \theta_{2}}{\left(1+B_{1}\right)}}_{\begin{array}{c}
\text { integral } \\
\text { feedback gain }
\end{array}} \underbrace{\int_{0}^{t}\left(\frac{\mu}{\theta_{2}}-x_{2}\left(t^{\prime}\right)\right) d t^{\prime}}_{\begin{array}{c}
\text { negative } \\
\text { integral feedback }
\end{array}}+\underbrace{\frac{1+B_{2}}{1+B_{1}} \theta_{1} z_{2}}_{\begin{array}{c}
\text { other } \\
\text { feedback }
\end{array}} .
$$

We can observe an integral feedback term and a second feedback term that is dependent upon $z_{2}$. The other feedback term becomes negligible and the integral term dominates the overall feedback if

$$
\frac{1+B_{2}}{1+B_{1}} \theta_{1} z_{2} \ll \theta_{1} z_{1}
$$

which can occurs if $1+B_{1}$ is sufficiently large or if $z_{2}$ is sufficiently small. The latter can be achieved via large $\eta$, which drives $z_{2}$ to a much lower concentration than $z_{1}$. For this case

$$
u=\theta_{1} z_{1}=\underbrace{\frac{\theta_{1} \theta_{2}}{\left(1+B_{1}\right)}}_{\begin{array}{c}
\text { integral } \\
\text { feedback gain }
\end{array}} \underbrace{\int_{0}^{t}\left(\frac{\mu}{\theta_{2}}-x_{2}\left(t^{\prime}\right)\right) d t^{\prime}}_{\begin{array}{c}
\text { negative } \\
\text { integral feedback }
\end{array}}
$$

where we can observe that the feedback gain is dependent on $B_{1}$.

\section{S14.2. Characterisation with Non-rapid $z_{1}$ Buffering}

For the case of non-rapid $z_{1}$ buffering, we have

$$
\begin{aligned}
\dot{x}_{1} & =\theta_{1} z_{1}-\gamma_{p} x_{1} \\
\dot{x}_{2} & =k x_{1}-\left(\gamma_{p}+B_{x} \gamma_{x}\right) x_{2} \\
\dot{z}_{1} & =\mu-\eta z_{1} z_{2}-b_{z} z_{1}+b_{w} w \\
\dot{z}_{2} & =\theta_{2} x_{2}-\eta z_{1} z_{2} \\
\dot{w} & =b_{z} z_{1}-b_{w} w
\end{aligned}
$$

We have

$$
\dot{z}_{1}(t)+\dot{w}(t)-\dot{z}_{2}(t)=\mu-\theta_{2} x_{2}
$$

where the integral is

$$
z_{1}(t)+w(t)-z_{2}(t)=\theta_{2} \int_{0}^{t}\left(\frac{\mu}{\theta_{2}}-x_{2}\left(t^{\prime}\right)\right) d t^{\prime}
$$

and so perfect adaptation occurs. Rearranging, we have the feedback

$$
u=\theta_{1} z_{1}=-\theta_{1} w(t)+\theta_{1} z_{2}(t)+\theta_{1} \theta_{2} \int_{0}^{t}\left(\frac{\mu}{\theta_{2}}-x_{2}\left(t^{\prime}\right)\right) d t^{\prime} .
$$


Taking Laplace transforms, we have

$$
U=\theta_{1} Z_{1}=-\theta_{1} W+\theta_{1} Z_{2}+\theta_{1} \theta_{2} \frac{1}{s}\left(\frac{\mu}{\theta_{2}}-X_{2}\right)
$$

where $Z_{1}, W, Z_{2}, X_{2}$ are the Laplace transforms of $z_{1}, w, z_{2}, x_{2}$. Taking the Laplace transforms of (S37), we also have

$$
s W=b_{z} Z_{1}-b_{w} W
$$

and so

$$
W=\frac{b_{z}}{s+b_{w}} Z_{1}
$$

Substituting, we have

$$
\theta_{1} Z_{1}=-\theta_{1} \frac{b_{z}}{s+b_{w}} Z_{1}+\theta_{1} Z_{2}+\theta_{1} \theta_{2} \frac{1}{s}\left(\frac{\mu}{\theta_{2}}-X_{2}\right)
$$

and so the control input is

$$
U=\theta_{1} Z_{1}=\theta_{1} \frac{s+b_{w}}{s+b_{z}+b_{w}} Z_{2}+\theta_{1} \theta_{2} \frac{s+b_{w}}{s+b_{z}+b_{w}} \frac{1}{s}\left(\frac{\mu}{\theta_{2}}-X_{2}\right)
$$

If $Z_{2}$ is small due to large $\eta$, then we have

$$
U=\theta_{1} Z_{1}=\theta_{1} \theta_{2} \frac{s+b_{w}}{s+b_{z}+b_{w}} \frac{1}{s}\left(\frac{\mu}{\theta_{2}}-X_{2}\right)
$$

Thus for non-rapid buffering (small $b_{w}$ ) we are able to add a zero and pole and zero to the controller, where the zero is small than the pole. This result is equivalent to the integral controller in series with a lead controller, where the latter is know to help stabilise systems ${ }^{1}$.

We can see the effect of non-rapid buffering on the integral component of feedback gain separately by determining the gain of the control input in the asymptote as $s \rightarrow 0$, where we have

$$
\theta_{1} \theta_{2} \frac{s+b_{w}}{s+b_{z}+b_{w}} \frac{1}{s}=\frac{\theta_{1} \theta_{2}}{1+B_{1}} \frac{1}{s}
$$

Thus the integral component of feedback gain is $\frac{\theta_{1} \theta_{2}}{1+B_{1}}$, which is identical to the rapid case.

\section{S14.3. Bode Integral of Buffering and Antithetic Integral Feedback}

Feedback is a highly effective method of robust regulation, but this mechanism is subject to fundamental limits. The Bode integral describes one of these fundamental limit, where improving the regulation at one frequency of a disturbance will worsen regulation of disturbances at other frequencies. Here, we show that topology 3 has the ability to reduce the fundamental limit on feedback and thus uniformly improve output regulation at all frequencies, while topology 1 does not. While we show the two state example from above to illustrate the concept, the Fourier transform description below is written generally as the Bode Integral holds for more general processes controlled by antithetic feedback and buffering.

We use the example model of the system

$$
\begin{aligned}
\dot{x}_{1} & =\theta_{1} z_{1}-\gamma_{p} x_{1} \\
\dot{x}_{2} & =k x_{1}-\gamma_{p} x_{2}-b_{x} w+b_{x} w_{x} \\
\dot{w}_{x} & =b_{x} x-b_{w} w_{x}-\gamma_{x} w \\
\dot{z}_{1} & =\mu-\eta z_{1} z_{2}-\gamma_{c} z_{1}-b_{z} z_{1}+b_{w} w_{1} \\
\dot{w}_{1} & =b_{z} z_{1}-b_{w} w_{1}-\gamma_{c} w_{1} \\
\dot{z}_{2} & =\theta_{2} x_{2}-\eta z_{1} z_{2}-\gamma_{c} z_{2} .
\end{aligned}
$$


We can write the linearised model in an open-loop form with the two states

$$
\begin{aligned}
& \Delta \dot{x}_{1}=\theta_{1} u_{h}-\gamma_{p} \Delta x_{1} \\
& \Delta \dot{x}_{2}=k \Delta x_{1}-\gamma_{p} \Delta x_{2}+u_{b}
\end{aligned}
$$

where $\Delta x_{i}=x_{i}-\bar{x}_{i}$ for $i=1,2, \bar{x}_{i}$ is the steady state of $x_{i}$ for the closed loop system, $u_{h}$ is the process input for antithetic integral feedback and $u_{b}$ is the input for buffering at $x_{2}$. Buffering at $z_{1}$ and antithetic integral feedback act through the input $u_{a}$ while buffering at $x_{2}$ acts through $u_{b}$.

If we take the Fourier transform, we can describe the open loop model in general terms by ${ }^{3}$

$$
X_{2}=G(i \omega) U_{h}+G_{b}(i \omega) U_{b}
$$

where $X_{2}, U_{h}, U_{b}$ are the Fourier transforms of $x_{2}, u_{h}, u_{b}$ respectively, $G$ is the transfer function from $U_{h}$ to the output $X_{1}$ and $G_{b}$ is the transfer function from $U_{h}$ to $X_{1}$. With two control inputs we have the loop transfer function ${ }^{3}$

$$
L(i \omega)=G(i \omega) C_{h}(i \omega)+G_{b}(i \omega) C_{b}(i \omega)
$$

where $C_{h}$ is the transfer function for the antithetic feedback controller (i.e. from $X_{2}$ to $U_{h}$ ) and $C_{b}$ is the transfer function for the output buffer (i.e. from $X_{2}$ to $U_{b}$ ). The sensitivity function quantifies the improvement or worsening in regulation at each frequency for the 'closed-loop' system, where a smaller magnitude implies improved regulation, and can be described by

$$
S(i \omega)=\frac{1}{1+L(i \omega)}
$$

In this case the sensitivity function incorporates the regulatory effect of both feedback and buffering ${ }^{3}$. The Bode integral is a fundamental constraint on the effectiveness of feedback in any system. It provides a constraint on the overall regulatory effectiveness in terms of the sensitivity function. If the system without feedback is stable then Bode's integral with output buffering (Topology 3) is ${ }^{3}$

$$
\int_{0}^{\infty} \log (|S(i \omega)|) d \omega=-\frac{\pi}{2} b_{x}
$$

where the integral of $S(i \omega)$ represents an overall measure of regulation and $b_{x}$ is the kinetic rate of the forward buffering reaction. The integral of $S(i \omega)$ sums the effect of oscillating disturbances at different frequencies $\omega$. Without buffering, if regulation is improved at one frequency of regulation, it worsens at other frequencies. However, increasing $b_{x}$ reduces the whole integral. Thus buffering can uniformly improve regulation and thus improve the trade-off.

In contrast, control species buffering (Topology 1) is part of the feedback regulation mechanism using the same control input and so Bode's integral is ${ }^{3}$

$$
\int_{0}^{\infty} \log (|S(i \omega)|) d \omega=0
$$

Thus control buffering does not remove fundamental constraints, despite stabilising buffering. The tradeoff remains such that improving regulation at one frequency will worsen regulation of disturbances at other frequencies.

\section{References}

${ }^{1}$ K. J. Aström and R. M. Murray. Feedback Systems. Princeton University Press, 2008.

${ }^{2}$ J. A. M. Borghans, R. J. De Boer, and L.A. Segel. Extending the quasi-steady state approximation by changing variables. Bull. Math. Biol., 58(1):43-63, 1996.

${ }^{3}$ E.J. Hancock and J.Ang. Frequency domain properties and fundamental limits of buffer-feedback regulation in biochemical systems. Automatica, 103:330-336, 2019.

${ }^{4}$ E.J. Hancock, J.Ang, A.Papachristodoulou, and G-B.Stan. The interplay between feedback and buffering in cellular homeostasis. Cell Systems, 5(5):498-508, 2017.

${ }^{5}$ N. Olsman, A-A. Baetica, F. Xiao, Y.P. Leong, R.M. Murray, and J.C. Doyle. Hard limits and performance tradeoffs in a class of antithetic integral feedback networks. Cell Systems, 9, 2019. 